BRUNEL

UNIVERSTYY

\title{
UNCERTAINTY MODELLING IN POWER SYSTEM STATE ESTIMATION
}

by

\author{
AbdulRahman K. Al-Othman
}

A Thesis Submitted for the Degree of

Doctor of Philosophy

Brunel Institute of Power Systems

School of Engineering and Design

Brunel University

August 2004 
This thesis is dedicated to my old man and the people of old Kuwait 


\begin{abstract}
As a special case of the static state estimation problem, the load-flow problem is studied in this thesis. It is demonstrated that the non-linear load-flow formulation may be solved by real-coded genetic algorithms. Due to its global optimisation ability, the proposed method can be useful for off-line studies where multiple solutions are suspected.
\end{abstract}

This thesis presents two methods for estimating the uncertainty interval in power system state estimation due to uncertainty in the measurements. The proposed formulations are based on a parametric approach which takes in account the meter inaccuracies. A nonlinear and a linear formulation are proposed to estimate the tightest possible upper and lower bounds on the states. The uncertainty analysis, in power system state estimation, is also extended to other physical quantities such as the network parameters. The uncertainty is then assumed to be present in both measurements and network parameters. To find the tightest possible upper and lower bounds of any state variable, the problem is solved by a Sequential Quadratic Programming (SQP) technique.

A new robust estimator based on the concept of uncertainty in the measurements is developed here. This estimator is known as Maximum Constraints Satisfaction (MCS). Robustness and performance of the proposed estimator is analysed via simulation of simple regression examples, D.C. and A.C. power system models. 


\section{Acknowledgements}

There is no way to properly estimate the appreciation I owe to those who shaped and supported my life over the past years as I endeavoured to produce this piece of work and to graduate from the Brunel University with a PhD. Foremost in my mind are friends and family, without whom life would be a dreary process indeed.

I would first like to thank my supervisor, Professor Malcolm Irving. Beside being a great teacher, the advice I've received has been beneficial. I especially appreciate the tremendous effort he makes to yield his attention to his students and his guidance,

support, and unwavering patience through out my studies. I truly believe that the level of my knowledge has been significantly improved simply because he demanded and expected nothing less. To that I am forever grateful.

My thanks also to the members of my $\mathrm{PhD}$ examination committee, Professor K. L. Lo and Dr. Gary Taylor, for their valuable time in evaluating this thesis.

Of course, there are many other people that must be given their due: fellow graduate students and my office mates at Brunel Institute of Power Systems. My thanks to all the staff of Brunel institute of power system, especially to Dr. Jeremy Denial who has been very helpful to ensure that computer system is up and running. 
On the home front, I would like to thank my close friends at the "Duwaneya" in particular Majed Al-Majed, Hussien and Fahad Al-Sennawi, Rowdan Al-Rowdan, Jamal AlSumait, Dr. Hameed Khalaf, Dr. Mohamed Al-Failakawi and Mohamed Alhajri for bearing me the overseas calls and making me feel right at home. To Fowzan Al-Faris, and Mahdi Al-Ajmi, I thank them for their friendship and wish them best of luck with their studies. I owe a great debt to Professor Khaled El-Naggar, form university of Ain Shams-Cairo, who have inspired, advised and guided me in research prior to pursuing graduate studies.

The financial support of the Embassy of Kuwait-Cultural Attaché Office is greatly acknowledged. Special thanks are due to Mr. Saif Basher, Mr. Edrees and Mr. Sattar for their efforts and help to make this work possible.

Last, but not least, to my parents, brothers (Waleed \& Feras), kids, and whole extended family (my cousin Nasser Al-Othman in particular). Thank you for your constant support and encouragement throughout the many years of education and for helping to see the many opportunities. I sincerely appreciate the constant support of my truly great wife. Thanks for spurring me on when my energies were draining. 


\section{Declaration}

The work in this thesis has not been previously submitted for a degree in this or any other university and unless otherwise referenced it is the author's own work. 


\section{Statement of Copyright}

The copyright of this thesis rests with the author.

No quotation from it should be published without his prior consent, And information derived from it should be acknowledged.

C COPYRIGHT BY ABDULRAHMAN AL-OTHMAN 2004 All Rights Reserved 


\section{Table of Contents}

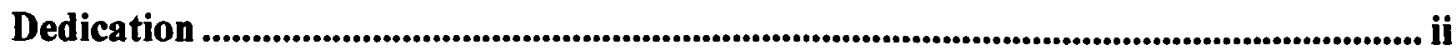

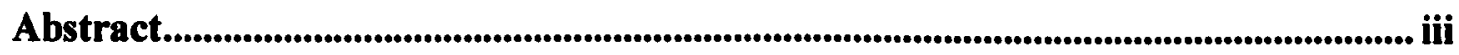

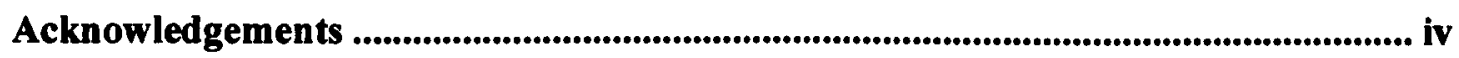

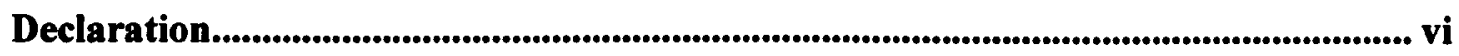

Statement of Copyright ............................................................................................... vii

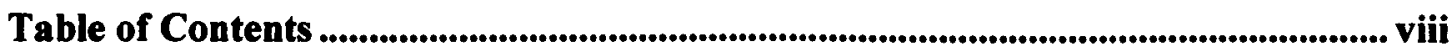

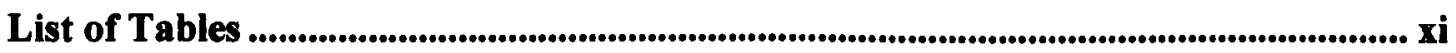

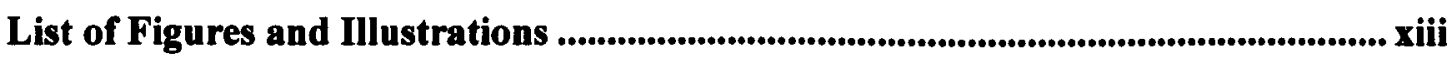

CHAPTER ONE: INTRODUCTION ......................................................... 1

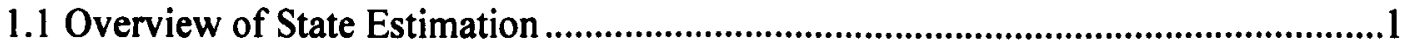

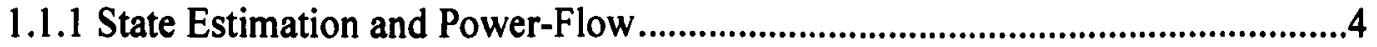

1.1.2 State Estimation and Power Market................................................................5

1.1.3 State estimation and the 2003 Blackout........................................................5

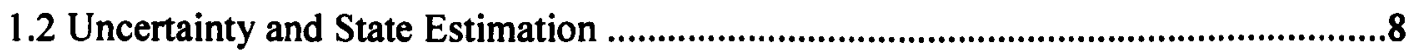

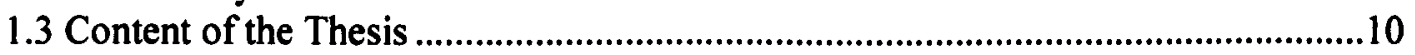

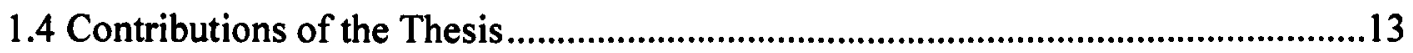

\section{CHAPTER TWO: LOAD FLOW ANALYSIS WITH REAL-CODED} GENETIC ALGORITHMS........................................................................... 15

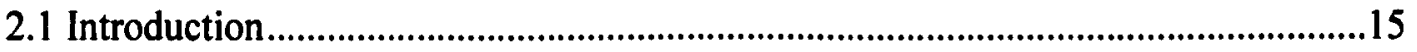

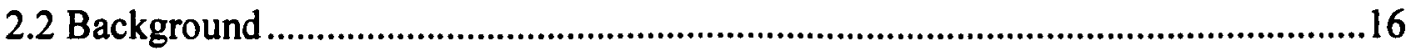

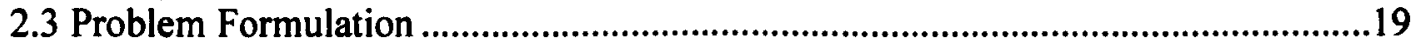

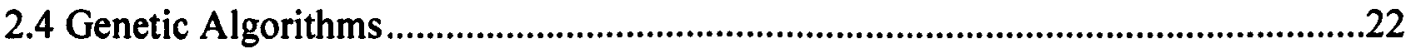

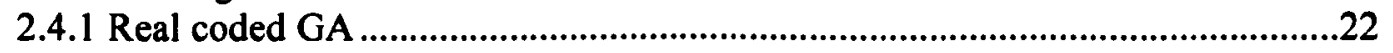

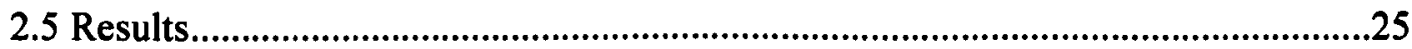

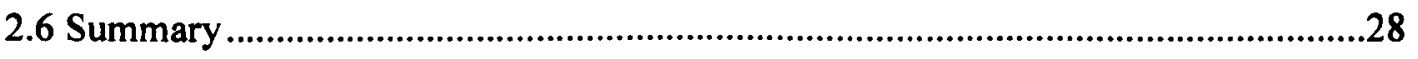




\section{CHAPTER THREE: ANALYSIS OF POWER SYSTEM STATE ESTIMATION WITH UNCERTAIN MEASUREMENTS.................... 29}

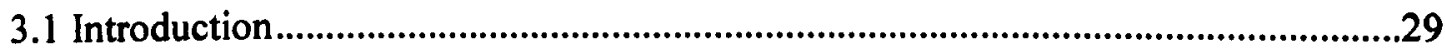

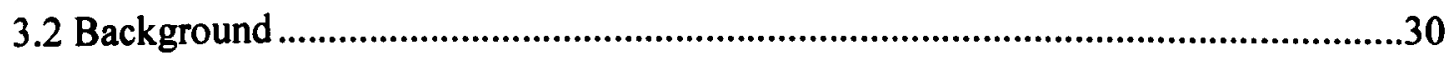

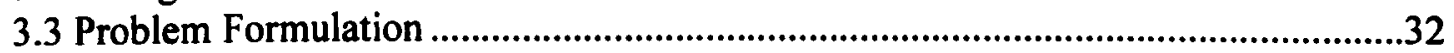

3.3.1 Weighted Least Square (WLS) ............................................................................32

3.3.2 Uncertainty interval estimation via linear programming (UILP) ........................34

3.3.3 Uncertainty interval calculation for other quantities: ...........................................36

3.4 Comparison of Two Formulations ..........................................................................39

3.5 Implementation of Case Studies and Results Analysis...............................................42

3.5.1 Six Bus System ...............................................................................................43

3.5.2 IEEE 30-bus test System ......................................................................................48

3.5.3 IEEE 118-bus test System...........................................................................51

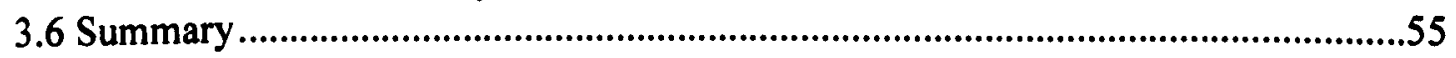

CHAPTER FOUR: ANALYSIS OF POWER SYSTEM STATE ESTIMATION WITH UNCERTAIN MEASUREMENTS AND

PARAMETERS............................................................................................... 57

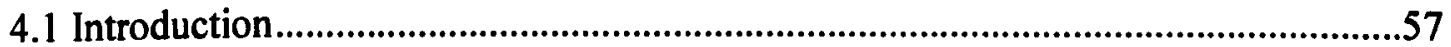

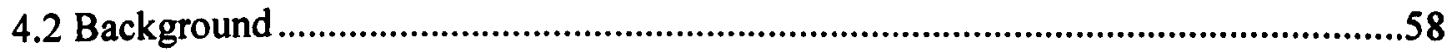

4.3 Proposed Formulation......................................................................................................61

4.4 Overview of non-linear optimization:....................................................................64

4.4.1 Sequential Quadratic Programming (SQP) .......................................................66

4.5 Results and analysis ..................................................................................................67

4.5.1 Estimation of states confidence bounds ...............................................................68

4.5.2 Estimation of confidence bounds on parameters ..................................................74

4.5.3 Estimation of other quantities ..........................................................................76

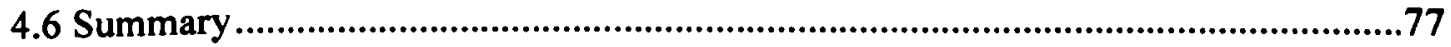

\section{CHAPTER FIVE: ROBUST STATE ESTIMATION ............................. 78}

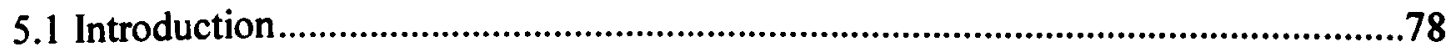

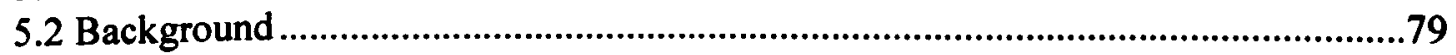

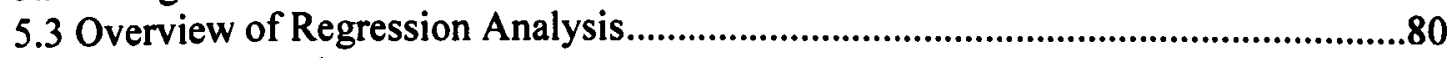

5.4 Breakdown Points .........................................................................................................

5.5 Overview of robust regression ...................................................................................88

5.5.1 Robust regression methods ................................................................................88

5.5.2 Regression diagnostic methods ........................................................................94

5.6 The shortcoming of the LMS estimator.................................................................97

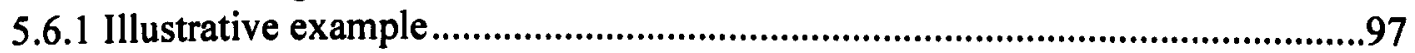

5.7 The Maximum Constraints Satisfaction Estimator ..................................................100

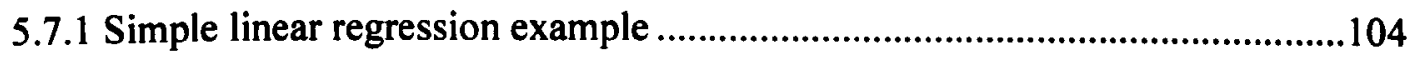




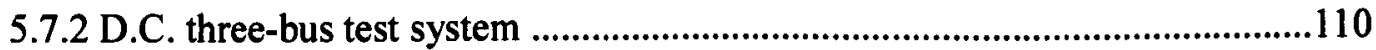

5.7.3 Six bus A.C. example...............................................................................115

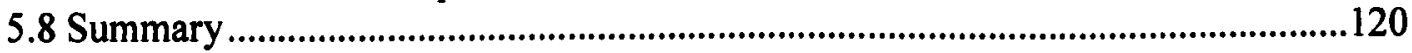

CHAPTER SIX: CONCLUSION_........................................................ 121

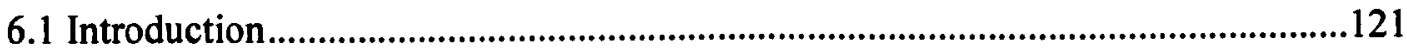

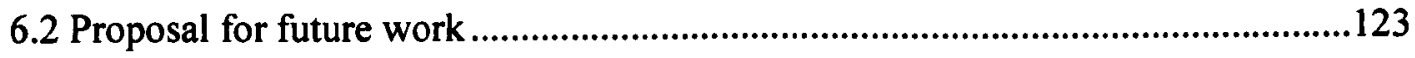

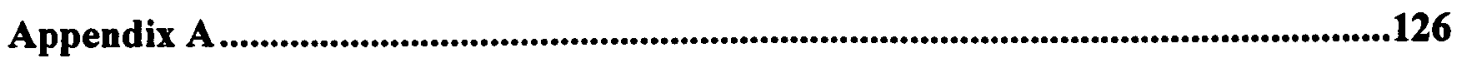

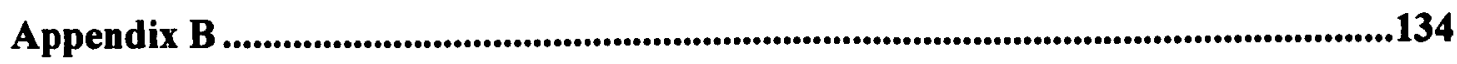

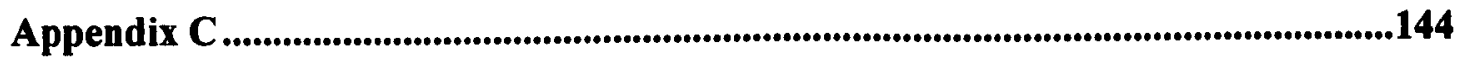

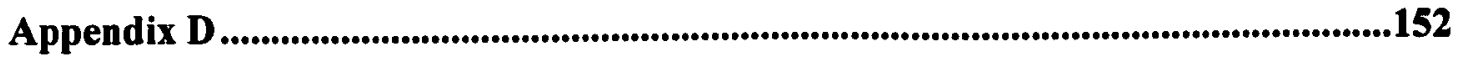

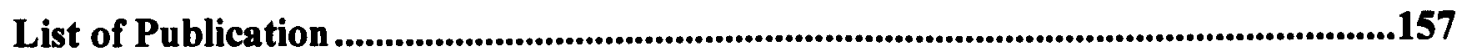

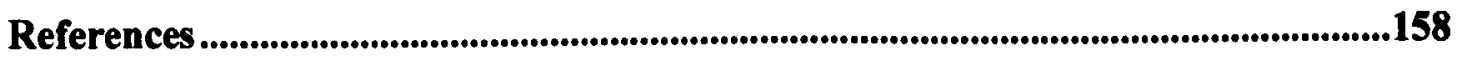




\section{List of Tables}

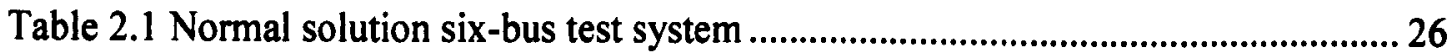

Table 3.1 Estimated state variables of the 6-bus system with redundancy $\approx 2 \ldots \ldots \ldots \ldots . . . . .40$

Table 3.2 Execution time of (WLS-LP) and (SQP) ...................................................... 41

Table 3.3 Estimated state variables and uncertainty bounds for the six-bus network ...... 43

Table 3.4 CPU and execution time of six-bus network …….........................................45

Table 3.5 Estimated measurements with uncertainty bounds ........................................... 46

Table 3.6 Estimated state variables and uncertainty bounds for the IEEE 30-bus network.

Table 3.7 CPU and execution time of IEEE 30-bus system ............................................51

Table 3.8 Estimated state variables and uncertainty bounds for the IEEE 118-bus network 52

Table 3.8 Estimated state variables and uncertainty bounds for the IEEE 118-bus network

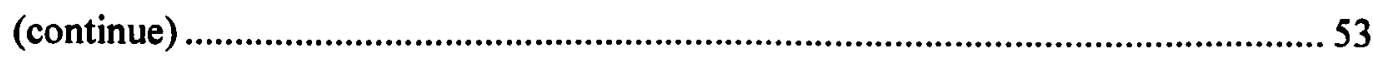

Table 3.8 Estimated state variables and uncertainty bounds for the IEEE 118-bus network

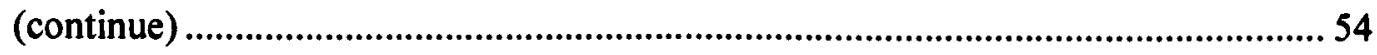

Table 3.8 Estimated state variables and uncertainty bounds for the IEEE 118-bus network

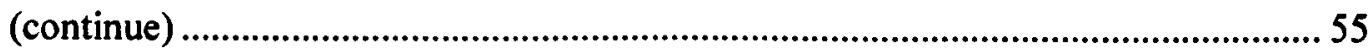

Table 4.1 Estimated state variables with uncertain parameters for the six-bus system .... 70

Table 4.2 Estimated states with uncertain measurements and parameters .......................... 71

Table 4.3 The estimated bounds of the IEEE 14-bus system .............................................. 73

Table 4.4 The estimated bounds of the IEEE 30-bus system ........................................... 73

Table 4.5 Estimated parameter bounds for the 6-bus system ............................................ 75 
Table 4.6 Estimated bounds of injection and flows of the 6-bus system.......................... 76

Table 4.7 Parameters of transmission line connecting bus 3 and bus $6 \ldots \ldots \ldots \ldots \ldots \ldots \ldots \ldots \ldots . . . . . . . . . . . . .76$

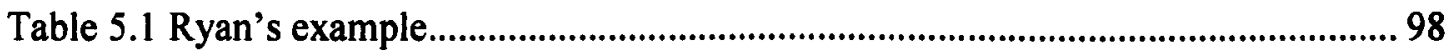

Table 5.2 Outcome of MCS estimator with single outlier in the measurement set ........ 117

Table 5.3 Outcome of MCS estimator with two outliers in the measurement set .......... 118

Table C.1 Operating Conditions for six-bus network system ........................................ 144

Table C.2 Impedance and Line Charging Data six-bus network system ........................ 144

Table C.3 Impedance and Line Charging Data of IEEE 14-bus system........................ 145

Table C.4 Impedance and Line Charging Data of IEEE 30-bus system........................ 145

Table C.4 Impedance and Line Charging Data of IEEE 30-bus system (continue) ....... 146

Table C.5 Impedance and Line Charging Data of IEEE 118-bus system...................... 147

Table C.5 Impedance and Line Charging Data of IEEE 118-bus system (continue) ..... 148

Table C.5 Impedance and Line Charging Data of IEEE 118-bus system (continue) ..... 149

Table C.5 Impedance and Line Charging Data of IEEE 118-bus system (continue) ..... 150

Table C.5 Impedance and Line Charging Data of IEEE 118-bus system (continue) ..... 151 


\section{List of Figures and Illustrations}

Figure 1.12003 blackout sequence of events .................................................................

Figure 2.1 Three-dimensional plot of the objective function $g(E, F)$............................... 21

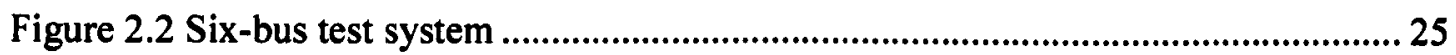

Figure 2.3 Real-Coded GA convergence behaviour of the six-bus test system................. 27

Figure 3.1 Two-dimensional example of LP uncertainty estimation................................... 38

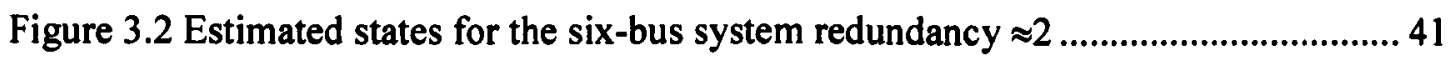

Figure 3.3 Estimated states and uncertainty bounds for the six-bus system....................... 44

Figure 3.4 Estimated states and uncertainty bounds for the six-bus network.................... 45

Figure 3.5 the estimated measurements with uncertainty bounds for six-bus system ...... 47

Figure 3.6 Measurements Bounds................................................................................ 48

Figure 4.1 LFT representation where all the uncertainties isolated in $\Delta$........................... 60

Figure 4.2 IEEE 14-bus test system .................................................................................. 68

Figure 5.1 Least squares regression with no outliers...................................................... 82

Figure 5.2 Least squares regression with outliers in the $\mathrm{x}$-direction .................................. 83

Figure 5.3 Least squares regression with outliers in the direction...................................... 84

Figure 5.4 The LMS strip covering half of the measurements. ........................................ 91

Figure 5.5 LMS result of two outliers in the $x$-direction ................................................... 92

Figure 5.6 LMS result of two outliers in the y-direction ................................................... 93

Figure 5.6 Mahalanobis distance (dashed ellipse) verses Robust distance (dashed ellipse)

Figure 5.8 Illustration of LMS shortcoming ................................................................... 99

Figure 5.9 The Maximum Constraints Satisfaction …………….................................... 102 
Figure 5.10 Application of MCS with outliers in the $x$-direction

Figure 5.11 Application of MCS with outliers in the $y$-direction .................................. 107

Figure 5.12 Application of MCS on Stefanski's example …….................................... 108

Figure 5.13 Stefanski's example without outliers..................................................... 109

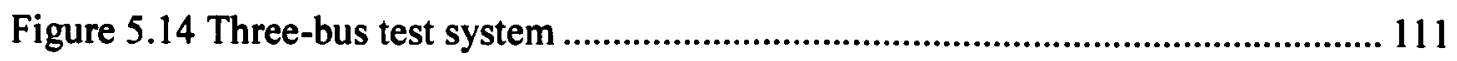

Figure 5.15 MCS performance with single bad data .................................................. 112

Figure 5.16 MCS performance with multiple interacting bad data ................................ 113

Figure 5.17 MCS performance with multiple interacting conforming bad data.............. 114

Figure 5.18 Number of satisfied constraints: six-bus test with no bad data .................... 115

Figure 5.19 Number of satisfied constraints: six-bus test with a single bad data ............ 116

Figure 5.19 Number of satisfied constraints: six-bus test with two bad data .................. 119

Figure A.1 Adaptive range GAs ......................................................................... 128

Figure A.2 Fitness function.................................................................................. 132

Figure A.3 Designed variables................................................................................ 133

Figure B.1 Wye-Wye transformer with neutral impedance on primary and secondary side

Figure B.2 corresponding primitive network. 137 


\section{CHAPTER ONE: INTRODUCTION}

\subsection{Overview of State Estimation}

Power system activities such as planning, decision making, operational control and online monitoring are necessary for secure operation. In order to carry out these activities properly, technical information on the system must be obtained by estimation. Both mathematical models and measurement are used to obtain these estimates. Ever since Schweppe et al. introduced state estimation more than three decades ago in [1-3], state estimation for the real-time modeling of the electric power system has remained an exceptionally active area of research. Thus far, there have been more than a thousand research and development publications on innovative and improved techniques, for further improvement of power system state estimation. More recently, such efforts have even advocated new approaches such as, dynamic, distributed, and non-WLS (Weighted Least Squares).

State estimation may be defined as a digital processing procedure that calculates a set of system states from a set of redundant measurements and applicable physical laws in an optimal way. Normally, having accurate estimation of the system's state variables is sufficient to comprehensively monitor the system operating conditions. Usually, the input information is classified as:

- Real time telemetered measurements of certain quantities of the system. 
- The mathematical model of the system and its instrumentation.

- Prior knowledge of some of the system measurements known as pseudomeasurements.

Pseudomeasurements are introduced as a form of redundancy. They are old measurements which are obtained from historical data, load forecasts, generation trends and data provided by neighboring grids. The inclusion of pseudomeasurements has a crucial effect on the estimation procedure, since the number of actual telemetered measurements is probably less than the states (unknowns) in large networks. Ultimately, such redundancy in the measurements allows for smoothing out of the gross noise in the measurements, and therefore reducing the estimation error. Examples of pseudomeasurements are known voltages and other settings of regulators, known generation and load trends and transaction schedules, and load forecasts.

The traditional objective of state estimation is to reduce the effects of measurement errors by utilizing the redundancy available in most measurement systems. In particular, the objective is to reduce the variance of the estimates and improve their overall accuracy. There are other major objectives of traditional state estimation:

- Detection of erroneous measurements and bad data.

- Detection of erroneous assumptions about the system, particularly the status of switches and breakers. 
- Use of redundancy in order to improve the parameters for the electrical models of the system.

Power system state estimation usually employs more measurements than the minimum number necessary to completely define the state of the system. Availability of measurement redundancy is very crucial for improving the quality of the estimate by detecting erroneous (bad data) measurements that may be inadvertently included in a given set. Broadly, the estimation process is formulated as an optimization problem and the system state variables are estimated by maximizing or minimizing a selected criterion. For electric power systems, the fundamental basis for most state estimators is the method of least squares. Schweppe et al. in [1-3] have laid out the main framework of static state estimation in electric power system, in which the residual vector is minimized in a least squares sense. The handling of measurements, however, was proposed such that a single batch of measurements is processed. Larson et al. have described, on the other hand, a state estimator configuration that is suitable for on-line and real-time processing of measurements $[4,5]$. Their method was also based on least squares. An obvious advantage of models advocated in [1-5], is that pseudomeasurements can be used together with real-time measurements for the purpose of reducing the metering and communication cost.

Generally state estimation may be categorized according to optimization criterion used in the objective function (minimization / maximization), which is usually formulated as either quadratic, i.e. least squares and least mean squares, or non-quadratic such as least absolute value. A great deal of papers on power system state estimation has employed variants of least squares and least absolute value, including a constrained formulation of 
them. Equality constraints have long been used in weighted least squares (WLS) estimators $[6,7]$. Abur and Celik [8] have reformulated and solved a constrained WLS using an interior point method. Both equality and inequality constrained least absolute value (LAV) criterion had been adopted by [9-11] for estimation. Another example of adoption of a non-quadratic criterion was suggested by Irving et al. , in [12], in which the problem is formulated as a linear programming of the sum of the least absolute values.

\subsubsection{State Estimation and Power-Flow}

The state estimation model is closely related to load-flow analysis, nevertheless, the main reason for introducing state estimation to large scale power systems was to deal with the many uncertainties associated with traditional load-flow calculations for a real electric system using real-time telemetered readings [1]. Uncertainty emerges due to communication errors, unexpected operational system changes, and errors in the mathematical model assumed. Other sources of uncertainty in measurements will be discussed in a subsequent section. The load-flow study of an electric power system is also known as "power-flow" study. In essence, this study involves the calculation of line loading given the generation and demand levels. In comparison, the classical load-flow employs a minimal set of the accurate measurements to determine the true state of the system. On the other hand, power system state estimation employs a larger set of redundant and possibly contaminated, measurements in order to estimate the true state of the electrical system. 
As a matter of fact, load-flow may be considered as a special case of state estimation. As an example, when the number of measurements used in a particular state estimation problem is equal to the number of unknowns, i.e. there is no redundancy, then the problem is equivalent to the A.C. load-flow problem [4]. In fact, the load-flow program is considered as an important element of the on-line state estimation model advocated by Larson et al. in [5]. In their proposed model, a load-flow solution is carried out, (based on exact set of measurements), and is utilized as an initial starting point for the on-line estimator.

\subsubsection{State Estimation and Power Market}

Without any doubt, state estimation plays a vital role in the emerging scenarios of the deregulation of the electric power industry. Many market decisions will be based on knowing the present state of the system accurately. Proper operation and monitoring of the power market, reliably and accurately, would be impossible without state estimators. Real-time power markets can not drive their valuations of real time information without having precise and ongoing information of almost all voltages and all flows in the electric network at all times.

\subsubsection{State estimation and the 2003 Blackout}

On August 14, 2003, large portions of the Midwest and Northeast United States and Ontario, Canada, experienced an electric power blackout. The outage affected an area 
with an estimated 50 million people and 61,800 megawatts (MW) of electric load in the states of Ohio, Michigan, Pennsylvania, New York, Vermont, Massachusetts, Connecticut, and New Jersey and the Canadian province of Ontario. The blackout began a few minutes after 4:00 pm Eastern Daylight Time (16:00 EDT), and power was not restored for 2 days in some parts of the United States. Parts of Ontario suffered rolling blackouts for more than a week before full power was restored. In three minutes, 21 power plants shut down, including 10 nuclear plants. An area of 9,300 square miles in the U.S. and Canada were without power.

According to the final report of United States \& Canada outage task force [13], a number of violations of North American Electric Reliability Council (NERC), Reliability Standards were committed:

1. Following the outage of the Chamberlin-Harding 345-kV line, FirstEnergy did not take the necessary actions to return the system to a safe operating state within $\mathbf{3 0}$ minutes.

2. FirstEnergy did not notify other systems of an impending system emergency.

3. FirstEnergy's state estimation/contingency analysis tools were not used to assess the system conditions.

4. FirstEnergy operator training was inadequate for maintaining reliable operation.

5. The Midwest ISO did not notify other reliability coordinators of potential problems.

6. The Midwest ISO did not have adequate monitoring capability. 
Generally, the primary functions of Independent System Operator (ISO) is to manage in real time and on a day-ahead basis the reliability of the bulk power system and the operation of wholesale electricity markets within their footprint.

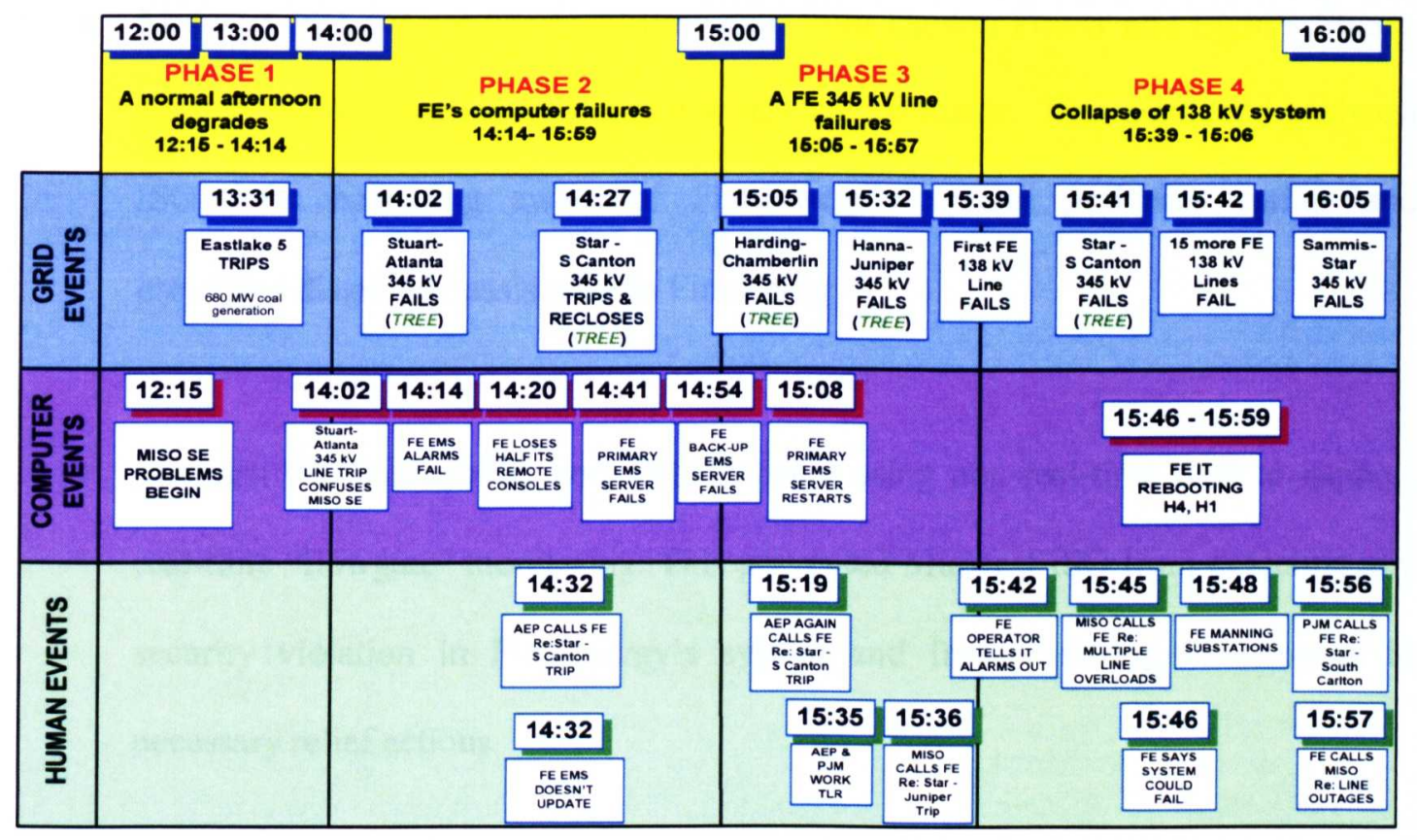

Figure 1.1 2003 blackout sequence of events

Figure 1.1 (which was derived form reference [14]) describes the sequence of events that led to 2003 blackout. Clearly, the initial problem was related to the state estimation procedure, in which the Midwest ISO state estimator and contingency analysis was ineffective from 12:37 to $16: 04$. In fact the state estimator was not functioning due to missing information on transmission line outages in Cinergy then DPL [13]. Also, human error made a significant contribution to the problem, which was not resetting a state estimation automatic trigger when they were required to do so. 
Among the primary causes of the 2003 blackout was the inadequacy of diagnostic support. This primary cause is explained as:

- Midwest ISO did not have real-time data from Dayton Power and Light's StuartAtlanta $345-\mathrm{kV}$ line incorporated into its state estimator. This precluded Midwest ISO from becoming aware of FirstEnergy's system problems earlier and providing diagnostic assistance to FirstEnergy.

- Midwest ISO's reliability coordinators were using non-real-time data to support real-time "flowgate" monitoring. This prevented Midwest ISO from detecting any security violation in FirstEnergy's system and from assisting FirstEnergy in necessary relief actions.

\subsection{Uncertainty and State Estimation}

While state estimation is essential for reliable and secure operation of a power system, it must be emphasised that both mathematical models and measurements, used to estimate the state of the system, do not necessarily guarantee a relatively accurate estimation. That is because of modelling errors and the possible presence of erroneous measurements. As a consequence, uncertainty in the estimates obtained is inevitable. 
The uncertainty is a parameter associated with the measurement that describes the dispersion of the values that could reasonably be attributed to the measured quantity [15]. This uncertainty reflects the lack of complete knowledge of the exact value of the quantity being measured. Theoretically, availability of complete knowledge about the measured quantity requires an infinite amount of information, which is practically impossible. Phenomena that contribute to the uncertainty are called sources of uncertainty. In fact, there are various possible sources of uncertainty in a measurement [15], including:

- Incomplete definition of the measured quantity.

- Imperfect realization of the definition of the measured quantity.

- Non-representative sampling (the sample measured may not represent the defined measured quantity).

- Insufficiently known effects of environmental conditions or imperfect measurements of these.

- Personal bias in reading analogue instruments (human error).

- Finite instrument resolution or discrimination threshold.

- Inexact values of measurement standards and reference materials.

- Inexact values of constants and other parameters obtained from external sources and used in the data-reduction algorithm.

- Approximations and assumptions incorporated in the measurement method and procedure. 
Traditionally, this uncertainty is handled using probability theory. Problems arise, however, due to the possibly invalid underlying assumptions concerning the probabilistic model of uncertainty and to nonlinearities in the network model. As a result, the power system operator can be faced with estimates whose values cannot be robustly assessed.

\subsection{Content of the Thesis}

In conventional state estimation techniques the accurate knowledge of error statistics of transducers and metering equipments is very essential. Nonetheless, such knowledge is not precisely available, leading to less accurate estimates. Providing estimate bounds together with the point estimates provides additional information that can improve the overall quality of the estimation. The knowledge of limiting values or bounds that may apply to measured quantities, due to possible physical limitations or operational characteristics, would facilitate a problem formulation that enables computation of estimate bounds. Thus, the main theme of this thesis is to model the uncertainties associated with the measured quantities in a way that defines an interval (range) with respect to their nominal values. Such a range is governed by the tolerance (claimed accuracy provided by the manufacturer), of the measuring instrument. Ultimately, by utilizing appropriate mathematical programming techniques, the confidence intervals (or bounds) on the state variables are obtained. The thesis is organized as follows:

Chapter two investigates, as a special case of the state estimation problem, the traditional non-linear formulation of the load-flow problem. An improved real-coded genetic 
algorithm (RGA) has been implemented. The outcome shows that the real-coded (RGA) consistently finds better solutions than those obtained using a conventional Genetic Algorithms. The proposed method shows reliability, accuracy and repeatability in solving the power-flow problem.

In chapter three, two methods for estimating the uncertainty interval in power system state estimation are presented. A non-linear and a linear formulation are presented to estimate the tightest possible upper and lower bounds on the states. The performance of both formulations is compared in terms of estimating the bounds of the uncertainty interval. In addition, an assessment of time performance for both methods is carried out with varying measurement redundancy level on a small test system. The linear method is considered further and is implemented to perform various estimation scenarios to estimate uncertainty bounds of power system state variables on larger test systems. It is also shown in this chapter that the method has the ability to provide useful additional information for both metered and non-metered elements of the system. The effects of network parameter errors are also studied.

The uncertainty formulation can also be extended to other physical quantities such as the network parameters. In chapter four a parametric method for uncertainty analysis in power system state estimation is proposed. The uncertainty is present in both measurements and network parameters. To find the tightest possible upper and lower bounds of any state variable, the problem is solved by Sequential Quadratic Programming (SQP) techniques. 
In chapter five, the robust estimators developed in statistics are reviewed. The wellknown Least Median of Squares (LMS) robust estimator and its application in power systems are revisited. A comparison between the LMS and LS is carried out to illustrate robustness concepts in estimation. Effects of collinearity in the measurement on the robust LMS estimator are studied.

A new robust estimator based on the concept of uncertainty in the measurements is developed. This estimator is known as Maximum Constraints Satisfaction (MCS). Robustness and performance of the proposed estimator is discussed via simulated examples of simple regression examples, D.C three-bus system and the six-bus test system.

Chapter six concludes the thesis and suggests proposals for further studies and future work in the field. Information and raw data of all tests systems are included in the appendix section, together with an adaptive version of Genetic Algorithms. Also, included in the appendix section, is the modelling of an unbalanced three-phase transformer, which has been developed during the current research, and which would be important for possible future work extending state estimation methods to unbalanced three-phase networks. 


\subsection{Contributions of the Thesis}

- An enhanced genetic algorithm (GA) for solving the load-flow problem is presented. The minimization of the total mismatch of the power balance equations is carried out with the help of a real-coded genetic algorithm (RGA) for the first time. Test results suggests that the real-coded (RGA) consistently finds better solutions than the conventional Genetic Algorithms.

- The uncertainty in power system state estimation is studied. Uncertainty in the measurements is modelled via a parametric approach. Two methods for estimating the uncertainty interval in power system state estimation are presented. A constrained non-linear and a linear formulation are proposed to estimate the tightest possible upper and lower bounds on the states. The proposed formulations have the advantage of providing useful additional information for both metered and non-metered elements of the system.

- A study of the effects of uncertainty in the network parameters and in the measurements is carried out in a unified framework. A parametric approach for uncertainty analysis in power system state estimation is proposed. The non-linear optimization problem is solved by Sequential Quadratic Programming (SQP) techniques. 
- An alternative robust estimator based on the concept of uncertainty in the measurements is developed. This estimator is known as Maximum Constraints Satisfaction (MCS). Robustness and effectiveness of the proposed estimator is discussed via simulated examples, where collinearity in the measurement undermines the performance of conventional, well-known robust estimators. 


\section{CHAPTER TWO: LOAD FLOW ANALYSIS WITH REAL-CODED GENETIC ALGORITHMS}

\subsection{Introduction}

In this chapter an improved genetic algorithm (GA) solution of the load-flow problem is presented. In order to minimize the total mismatch of the power balance equations, a real-coded genetic algorithm (RGA) has been implemented. The method is illustrated by various tests on a six-bus system. The results confirm that the real-coded (RGA) consistently find better solutions than the conventional Genetic Algorithms do. The proposed method shows reliability, accuracy and repeatability in solving the power-flow problem. 


\subsection{Background}

The power flow study of an electric power system is also known as "load-flow" study. In essence, this study involves the calculation of line loading given the generation and demand level. Ward and Hale [16] are frequently credited for being the first to formulate the load-flow problem. This problem has been studied widely and solved with the help of various numerical iterative methods such as Gauss-Seidel and Newton-Raphson [17-20]. Even though these numerical methods are very popular due to their effectiveness in finding solutions, they suffer from two main problems. First, they may not be able to converge unless a good initial guess is provided. Second, these methods may get stuck at some local optima since they possess poor global search capability.

Optimization Algorithms such as Non-linear programming, quadratic programming, sequential unconstrained minimization techniques and interior point methods are possibly good candidates to solve the load-flow problem. Nonetheless, most of these approaches apply sensitivity or Gradient-based techniques to probe the optimum by calculating the local gradient information. Unfortunately, the load-flow problem is highly nonlinear and has more than one local optimum solution. Consequently, the optimum obtained from local optimization methods may not be a global one, particularly in the load-flow optimization.

Heuristic algorithms such as genetic algorithms have the ability to combat the above drawbacks. As an optimization technique, genetic algorithms [21, 22] are much less 
dependent on the start values of the variables in the optimization problem when compared with the widely used Newton-Raphson or mathematical programming techniques such as SQP (Sequential Quadratic Programming). In addition GAs do not rely on the guidance of the gradient information, such as the Jacobian matrix, hence they are more capable of determining the global optimum solution. GAs can deal with problems that are usually considered very hard by researchers, such as integer variables, non-convex functions, non-differentiable functions, domains not connected, badly behaved functions, multiple local optima, and multiple objectives $[23,24]$. For these reasons, GAs has been adopted in this study to solve the load-flow problem.

Converging to a global optimum in the continuous domain is challenging for GAs. In traditional GAs, binary representation has been used for chromosomes, which equally discretizes a real design space. While such binary-coded GAs have been effectively applied to a wide range engineering problems, binary-coded GAs suffer from disadvantages, when applied to the problems involving a large number of real design variables (see appendix D). Since binary substrings representing each parameter with the desired precision are concatenated to form a chromosome for the GAs, the resulting chromosome encoding a large number of design variables would result in a large string length. Further more, there exist an inconsistency between the binary representation space and the actual problem space. For instance, two points close to each other in the representation space might be far away (in terms Hamming distance) in the binary represented problem space. 
Yin and Germay were the first who applied GAs to solve the load-flow problem. Unfortunately weaknesses of the traditional GAs have led to results being not very near the solution. It was shown in [25] that the total mismatch (accuracy) achieved for three runs of GA on the six-bus test system were $1.0216,0.5356$ and 0.5218 . Apparently, these mismatches can only suggest that the solution is quite inaccurate and the problem remains to be solved. These inaccuracies were probably due to the binary representation of candidates, which led to discretization errors. Also, it has been conceded in [25] that those GA solutions can only serve as a guide within the solution search space. The GA solution could then be used as an initial guess for the Newton-Raphson method, which would hopefully converge to the exact solution.

Wong et al. introduced, in [26-28], a constrained GA for solving power-flow. This approach was based on a constraint satisfaction technique to force the mismatch of the total power balance equations to zero. By incorporating the concept of dependant variables in the formulation [28] and setting the mismatch to zero, the power injection equations are reorganized to solve for the unknowns (nodal phasor voltages). Using this reformulation of the loadflow equations, Wong et al. found that the GA could successfully converge to the correct solution.

One way to solve the problems posed by the conventional GAs is the use of floating point representation of parameters as a chromosome [22, 29], which is known as real-coded GAs. In these real-coded GAs, a chromosome is coded as a finite-length string of the real numbers corresponding to the design variables. The real-coded GAs are rigorous, 
precise, and efficient because the floating point representation is conceptually closest to the real design space, and additionally, the string length reduces to the number of design variables. A comparative study conducted by Janikow et al., in [30], has concluded that the real-coded GAs outperformed binary-coded GAs in many optimization problems.

The proposed RGA has the ability to overcome all the problems which confronted the binary coded GA. In this study an efficient RGA is employed to solve the conventional formulation of the load-flow problem. The RGA solution will be compared to that obtained from N-R to demonstrate the quality of solution and the robustness of RGA.

\subsection{Problem Formulation}

There are two well known expressions for load-flow computations. One is expressed in polar form, where unknowns take the form $\bar{V}=V e^{j \theta}$. And the second is expressed in rectangular, in which unknowns take the form $\bar{V}=E+j F$.

Interestingly, polar and rectangle expressions differ in their load-flow convergence characteristics. Analysis of load-flow convergence characteristics [31, 32] demonstrated that the rectangular co-ordinate formulation has better convergence characteristics in the vicinity of multiple solutions. Therefore, rectangular expressions for the load-flow are adopted in this study.

The load-flow rectangular formulation can be described as follows. Consider a network with total number of $N$ nodes (buses). At any bus $i$, the nodal active $P_{i}$ and reactive $Q_{i}$ are given by: 


$$
\begin{aligned}
P_{i} & =E_{i} \sum_{j=1}^{N}\left(G_{i j} E_{i}-B_{i j} F_{i}\right)+F_{i} \sum_{j=1}^{N}\left(G_{i j} F_{i}-B_{i j} E_{i}\right) \\
i & =1,2,3 \ldots \ldots ., N \\
Q_{i} & =F_{i} \sum_{j=1}^{N}\left(G_{i j} E_{i}-B_{i j} F_{i}\right)-E_{i} \sum_{j=1}^{N}\left(G_{i j} F_{i}-B_{i j} E_{i}\right) \\
i & =1,2,3 \ldots \ldots . ., N
\end{aligned}
$$

where $G_{i j}$ and $B_{i j}$ are the $(i, j)$ th element of the admittance matrix. $E_{i}$ and $F_{i}$ are the real and the imaginary parts of the voltage at bus $i$. Whether the bus $i$ is PV or PQ bus the mismatch in active and reactive powers, $\Delta P_{i}$ and $\Delta Q_{i}$ respectively, are given by:

$$
\begin{aligned}
& \Delta P_{i}=\left|P_{i}^{s p}-P_{i}\right| \\
& \Delta Q_{i}=\left|Q_{i}^{s p}-Q_{i}\right|
\end{aligned}
$$

in which $P_{i}^{s p}$ and $Q_{i}^{s p}$ are the prespecified active and reactive power injection levels of bus $i$. The unknown variables in the above formulation are the real and the imaginary parts of the voltages at PV and PQ buses respectively. It is essential to determine the values of the unknowns such that the mismatch in equations (2-1) and (2-2) are zero (ideally).

Apart from solving the load-flow problem by conventional methods, the problem can be viewed as an optimization problem, in which the objective function $g$ is to be minimized. The objective function can be defined as the sum of the squares of the power mismatches:

$$
\min g(E, F)=\sum_{i \in N_{P p}+N_{P Q}}\left|P_{i}^{s p}-P_{i}\right|^{2}+\sum_{i \in N_{P Q}}\left|Q_{i}^{s p}-Q_{i}\right|^{2}
$$


where $N_{P V}$ and $N_{P Q}$ are the total number of PQ and PV buses, respectively. It is essential to point out that the voltage magnitude on all PV buses are set floating in equation $2-5$.

Figure 2.1 illustrates the shape of the objective function $g(E, F)$, for an arbitrary bus $i$ and fixing all variables of other buses. It is obvious that the minimum is located at the very bottom of the basin shape plot. In fact the bottom of the basin is relatively flat, where conventional GA might be trapped and therefore produce an abnormal solution. An adjustment to the objective function can be introduced to force convergence toward the normal solution [33].

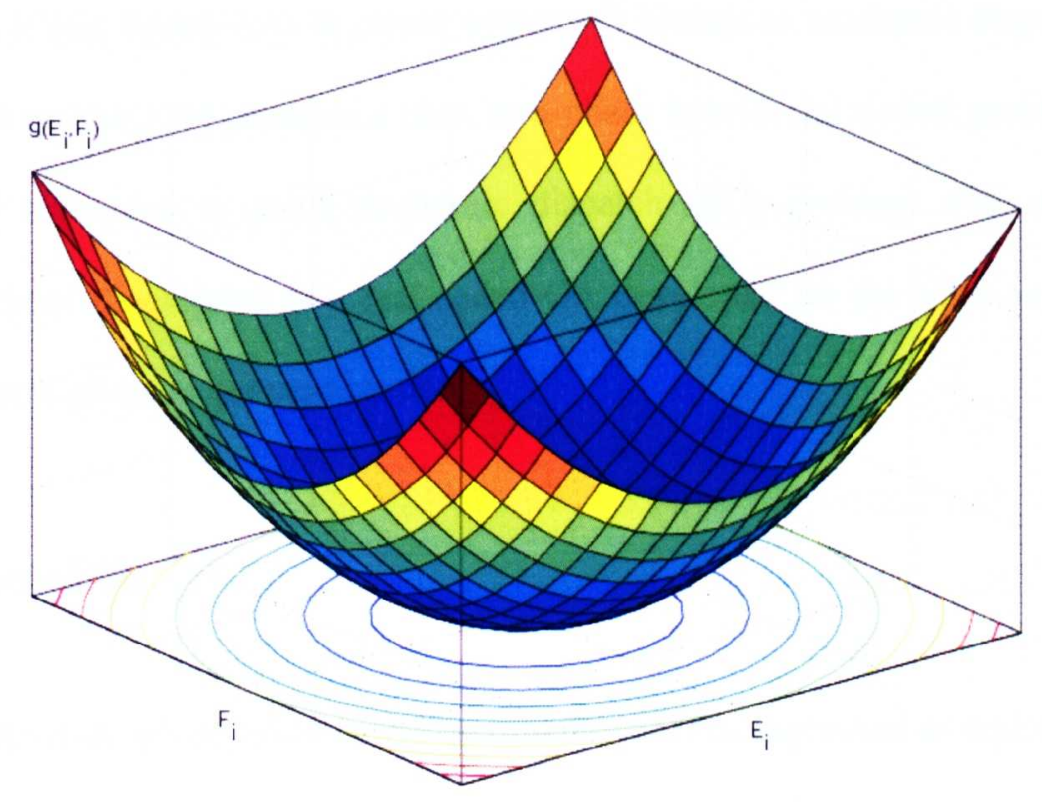

Figure 2.1 Three-dimensional plot of the objective function $g(E, F)$ 


\subsection{Genetic Algorithms}

GAs are inspired by the study of genetics $[21,22,34]$. They are conceptually based on natural evolution mechanisms working on populations of solutions. An interesting feature of GAs is that they do not require any prior knowledge of the solution and they tend to exhibit reliable performance on the majority of the problems [22].

Initially, GAs were designed to operate using binary representations of the problem parameters (or unknowns). In recent studies however, the superiority of higher cardinality alphabet GAs (floating point or integer) has been demonstrated with respect to their applications to various problems.

Application of real-coded GAs in power systems is limited to economic dispatch problem $[35,36]$. Reference [36] presents a new, two-phase hybrid real coded genetic algorithm (GA) based technique to solve economic dispatch (ED) problem with multiple fuel options. A brief description of a real-coded GA developed for the solution of the loadflow problem is given in the next section.

\subsubsection{Real coded GA}

In a real-coded $\mathrm{GA}$, all decision variables (unknowns) are expressed as real numbers (see appendix D). Explicit conversion to binary does not take place. A reduction of computational effort is an obvious advantage of real-coded GA. Another advantage is 
that an absolute precision is now attainable by making it possible to overcome the crucial decision of how many bits are needed to represent potential solutions.

As in a conventional GA, an initial population of chromosomes (potential solutions) is randomly created. The best size of this population is subject to experimentation with the problem at hand. Having created a population of chromosomes, it is possible to assess the performance, or fitness, of individual members of a population. This is done through an objective function (equation 2-5) that characterizes an individual's performance in the problem domain. Then a method known as ranking [37], is used to rank individuals according to their objective values. Based on that ranking (i.e. fitness) of each chromosome in the initial population, a selection scheme is carried out to pick the best individuals as members of the new generation.

The selection scheme used is known as Stochastic Universal Sampling [38]. This scheme, probabilistically selects individuals for reproduction according to their fitness. That is simply implemented by finding the cumulative sum of fitness of each chromosome in the population and generating equally spaced numbers between 0 and that sum. Therefore, only one random number is generated, all the others used being equally spaced from that point. The index of the chromosome selected is determined by comparing the generated numbers with the cumulative sum. The probability of an individual being selected is then given by

$$
F\left(x_{i}\right)=\frac{f\left(x_{i}\right)}{\sum_{i=1}^{N \text { ind }} f\left(x_{i}\right)}
$$


where $f\left(x_{i}\right)$ is the fitness of individual $x_{i}$ and $F\left(x_{i}\right)$ is the probability of that individual being selected.

A discrete recombination method (equivalent to crossover) is employed for mating individuals and breeding of offsprings. Discrete recombination exchanges variable values between the individuals. A method known as simple crossover $[22,39]$ is implemented. Specifically, let's assume that $C_{1}=\left(c_{1}^{1} \ldots c_{n}^{1}\right)$ and $C_{2}=\left(c_{1}^{2} \ldots c_{n}^{2}\right)$ are two chromosomes that are being subjected to crossover. A position $i \in(1,2,3, \ldots \ldots, n-1)$ is randomly assigned. The two new chromosomes are made as the following:

$$
\begin{gathered}
C_{1, \text { new }}=\left(c_{1}^{1}, c_{2}^{1}, \ldots, c_{i}^{1}, c_{i+1}^{2}, \ldots c_{n}^{2}\right) \\
C_{2, \text { new }}=\left(c_{1}^{2}, c_{2}^{2}, \ldots, c_{i}^{2}, c_{i+1}^{1}, \ldots c_{n}^{1}\right)
\end{gathered}
$$

Mutation of real-valued population is accomplished with the breeder genetic algorithm in [40]. Each variable is mutated with a probability by addition of small random values (size of the mutation step). The mutation step can be reduced as the algorithm evolves.

The proposed algorithm uses a generation gap and fitness-based reinsertion to implement an elitist strategy whereby the most fit individuals always propagate through to successive generations. For example, if G-gap $=90 \%$, then population_size $\times$ G-gap new individuals are produced at each generation. And then population_size $\times(G$-gap -1$)$ best chromosomes are copied intact from the parent generation to the new generation to 
complete the population size (i.e. fill the gap). According to [21], a better average fitness is attained with the adoption of elitist strategy.

\subsection{Results}

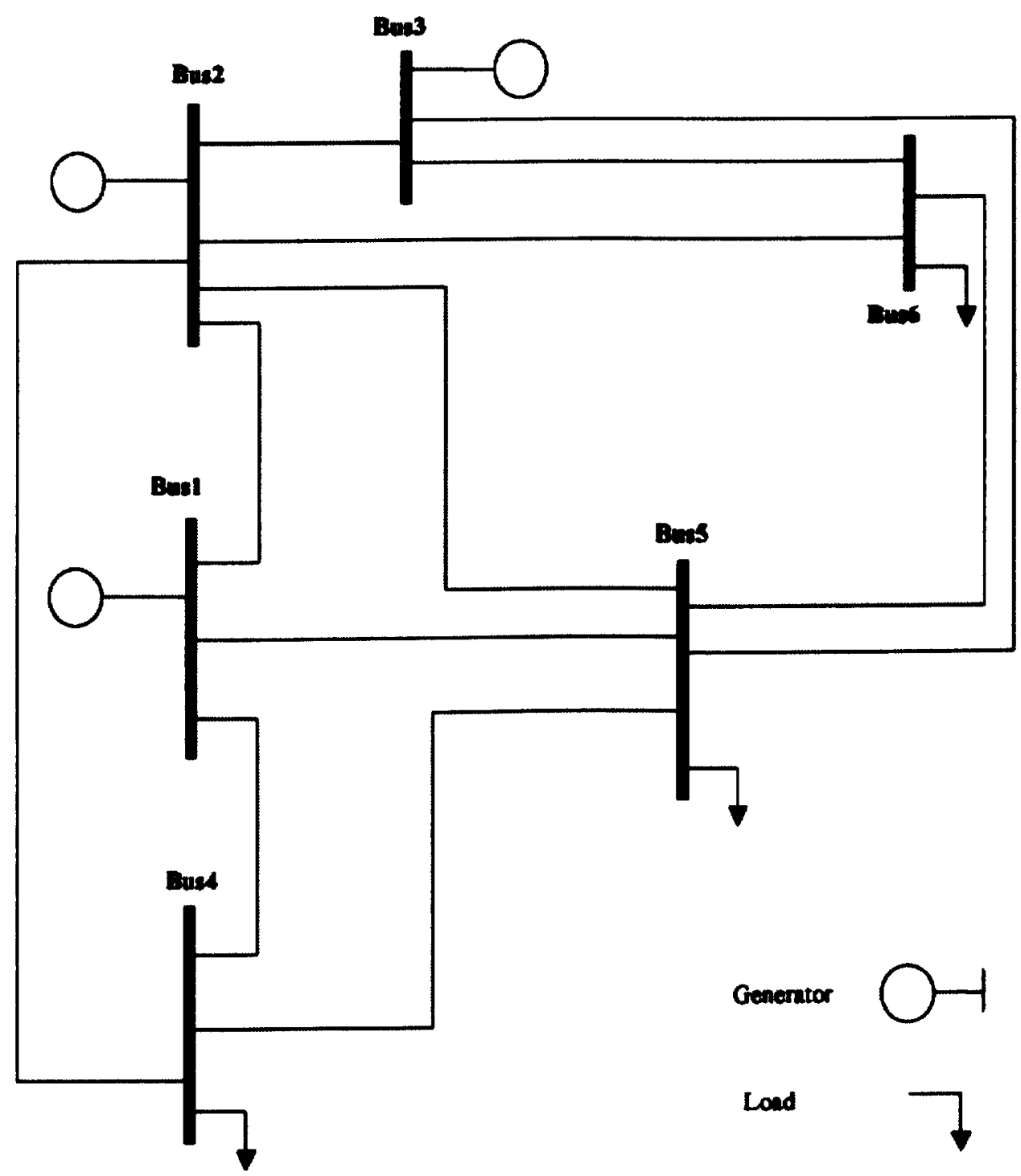

Figure 2.2 Six-bus test system 
To demonstrate the accuracy and the repeatability of the proposed method, RGA has been applied to the six-bus system from [41], shown in figure 2.2. The RGA parameters used in these tests are: population_size $=200$, mutation rate $=0.01$ and generation gap G-gap $=0.8$. To prove repeatability, the algorithm was executed forty times; table 2.1 shows results obtained from five runs of RGA. Solution of the same network was also carried out by Newton-Raphson for comparative purposes. Execution time and total squared mismatches have also been provided for each run.

Table 2.1 Normal solution six-bus test system

\begin{tabular}{|c|c|c|c|c|c|c|}
\hline \multirow{2}{*}{ Variable } & \multirow{2}{*}{ N-R } & \multicolumn{5}{|c|}{ Real-Coded RGA } \\
\cline { 3 - 7 } & & Run1 & Run1 & Run3 & Run4 & Run5 \\
\hline $\mathbf{F}_{\mathbf{1}}$ & 0 & 0 & 0 & 0 & 0 & 0 \\
\hline $\mathbf{F}_{\mathbf{2}}$ & -0.0682 & -0.0682 & -0.0682 & -0.0682 & -0.0682 & -0.0682 \\
\hline $\mathbf{F}_{3}$ & -0.0809 & -0.0808 & -0.0808 & -0.0808 & -0.0808 & -0.0808 \\
\hline $\mathbf{F}_{4}$ & -0.0719 & -0.0719 & -0.0719 & -0.0719 & -0.0719 & -0.0719 \\
\hline $\mathbf{F}_{\mathbf{5}}$ & -0.0892 & -0.0892 & -0.0892 & -0.0892 & -0.0892 & -0.0892 \\
\hline $\mathbf{F}_{6}$ & -0.1042 & -0.1041 & -0.1041 & -0.1041 & -0.1041 & -0.1041 \\
\hline $\mathbf{E}_{1}$ & 1.0500 & 1.0500 & 1.0500 & 1.0500 & 1.0500 & 1.0500 \\
\hline $\mathbf{E}_{2}$ & 1.0478 & 1.0478 & 1.0478 & 1.0478 & 1.0478 & 1.0478 \\
\hline $\mathbf{E}_{3}$ & 1.0669 & 1.0669 & 1.0669 & 1.0669 & 1.0669 & 1.0669 \\
\hline $\mathbf{E}_{4}$ & 0.9838 & 0.9838 & 0.9838 & 0.9838 & 0.9838 & 0.9838 \\
\hline $\mathbf{E}_{\mathbf{5}}$ & $\mathbf{0 . 9 7 5 6}$ & 0.9756 & 0.9756 & 0.9756 & 0.9756 & 0.9756 \\
\hline $\mathbf{E}_{6}$ & 0.9960 & 0.9960 & 0.9960 & 0.9960 & 0.9960 & 0.9960 \\
\hline Total Mismatch: & $9.27 \mathrm{e}-008$ & $3.61 \mathrm{e}-007$ & $1.31 \mathrm{e}-007$ & $1.38 \mathrm{e}-007$ & $9.78 \mathrm{e}-008$ \\
\hline Execution Time: & $2.981 \mathrm{~min}$ & $2.652 \mathrm{~min}$ & $3.297 \mathrm{~min}$ & $2.409 \mathrm{~min}$ & $2.980 \mathrm{~min}$ \\
\hline
\end{tabular}

Solution of the sates obtained by RGA for each of the five runs appears to be identical to those obtained by N-R. This shows that RGA is able to reproduce the normal solution, unlike the binary-coded GA which suffers from not being able to do so accurately. Also it is clearly shown that RGA can drive down the total squared mismatch to the order of $10^{-8}$ (in reasonable CPU time), while the smallest mismatch attained by binary-coded GA was 0.5218 for the Ward-Hale six-bus system in [25]. The execution time required by RGA seems to be approximately half that required by the binary-coded GA. 
It may be important to mention that the six-bus test system used in this study is relatively larger than the Ward-Hale six-bus system. In fact the test system from [41] has three generator bus and eleven branches, while the Ward-Hale six-bus system consists only of two generator bus and seven branches.

Figure 2.3 illustrates the convergence characteristics of the RGA for four of the tests in table 1. Variation in convergence of each run is apparent from the plot. It is evident that runl has the best convergence amongst these runs.

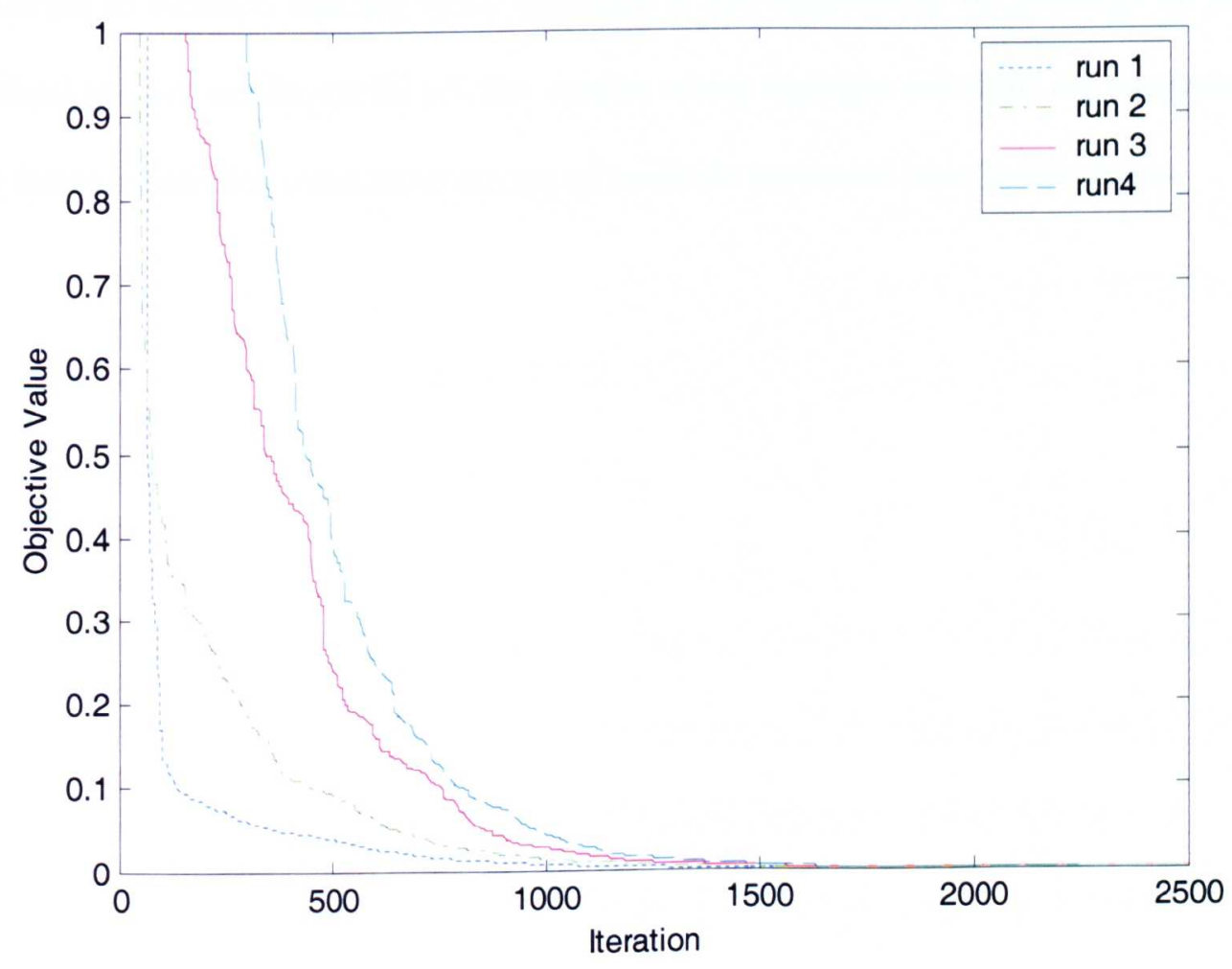

Figure 2.3 Real-Coded GA convergence behaviour of the six-bus test system 


\title{
2.6 Summary
}

\begin{abstract}
Although it has been claimed in the literature that GAs can only provide near optimal solution for the load-flow problem, this study has demonstrated that a real-coded GA is able to provide an exact solution to the problem. Various tests have been conducted on a six-bus test system. Results show that the proposed method was accurate, reliable, and in particular repeatable. The proposed RGA can be regarded as an efficient method when compared to other evolutionary methods for solving the load-flow problem. It is important to mention that the RGA approach is not expected to be practical for on-line applications, but can be useful off-line studies where multiple solutions are suspected and also forms a basis for some more advanced methods presented later in this thesis.
\end{abstract}




\section{CHAPTER THREE: ANALYSIS OF POWER SYSTEM STATE ESTIMATION WITH UNCERTAIN MEASUREMENTS}

\subsection{Introduction}

This chapter presents two methods for estimating the uncertainty interval in power system state estimation. The proposed formulations are based on a parametric approach. A non-linear and a linear formulation are proposed to estimate the tightest possible upper and lower bounds on the states. The non-linear formulation is solved by a Sequential Quadratic Programming (SQP) technique. The linear formulation relies upon two-step method that uses static weighted least-squares analysis to compute 'point' state estimates. Linear programming is then employed to obtain the upper and lower bounds of the uncertainty interval.

The performance of both formulations is compared in terms of estimating the bounds of the uncertainty interval. In addition, an assessment of time performance for both methods is carried out with varying measurement redundancy level on the six-bus test systems. Due to its superiority and efficiency, relative to the non-linear, the linear method is considered further and is implemented to perform various estimation scenarios to estimate uncertainty bounds of power system state variables. It is also shown that the method has the ability to provide useful additional information for both metered and non- 
metered elements of the system. The effects of network parameter errors are also studied. For illustrative purposes, the linear method is tested using the six-bus, IEEE 30-bus and IEEE 118-bus standard systems. Results show that the proposed methods are an accurate and reliable tool for estimating the uncertainty bounds in power system state estimation.

\subsection{Background}

The availability of an accurate picture of the system-state is an important aspect of power system operation. While a SCADA system is capable of providing operators with measured information, a state estimator has the ability to filter the available information creating a more accurate and complete picture of the system conditions. The traditional objective of state estimation is to reduce the effect of measurement errors by utilizing the redundancy available in the measurement system. In particular, the objective is to reduce the variance of the estimates and improve their overall accuracy. The other major objectives of state estimation methods include: detection of gross errors, detection of invalid topological information and detection of model parameter errors.

If the errors in the measurements follow a known probability distribution, then the set of feasible estimates can also be modelled by a probability distribution function. Unfortunately, the statistics of the observation errors are difficult to characterize in practice. In such circumstances, it is desirable to provide not just a single 'optimal' estimate of each state variable but also an uncertainty range within which we can be assured that the 'true' state variable must lie. The idea of an uncertainty range is 
recognizable in engineering practice, where the accuracy of a particular measurement is often described as (for example) plus or minus 2 percent, rather than by quantifying the standard deviation or variance.

Schweppe [42] introduced the concepts of uncertainty in the general context of engineering analysis, estimation and optimization. Schweppe proposed a new class of estimation problems called "unknown-but-bounded" estimation. As indicated by its name, unknown-but-bounded estimation does not assume any a priori knowledge of the measurement uncertainty. These concepts have been extended and developed recently and have been applied in a number of areas. The present author is not aware of any previous applications in power system state estimation. However, uncertainty estimation has been considered in the context of water distribution networks. Bargiela and Hainsworth [14] introduced bounds on the measurements, with an intention to increase the robustness of estimation. The approach was developed by Brdys and Chen [43], who introduced the term Set Bounded State Estimation (SBSE). Anderson et al [44] formulated a variant of WLS state estimator, in which the measurement bounds are incorporated into a cost function. That procedure is termed maximally constrained weighted least square estimator (M-WLS).

The most widely adopted techniques for power system state estimation are based on weighted least squares (WLS). Some efficient algorithms for solution of the WLS subproblem are given by Bjorck $[45,46]$. Hitherto, no research seems to have been conducted on uncertainty interval analysis for power system state estimation. This 
chapter propose two methods for estimating the uncertainty interval around the system state variables.

\subsection{Problem Formulation}

\subsubsection{Weighted Least Square (WLS)}

WLS is the most popular method of point estimation. For a set of measurement equations:

$$
\underline{z}=\underline{h}(\underline{x})+\underline{\varepsilon}
$$

where:

$\underline{z} \quad$ is the $(m x 1)$ measurement vector.

$\underline{h} \quad$ is a vector of non-linear functions that relate the states to the measurements.

$\underline{x} \quad$ is an $(n x 1)$ state vector to be estimated.

$\underline{\varepsilon} \quad$ is an $(m \times 1)$ measurement error vector.

The measurements are usually obtained from transducers in the electrical network. For observability, it is necessary that $m \geq n$ and that the $m$ measurements are in locations such that the resulting Jacobian (sensitivity matrix with respect to the state variables) has rank $n$.

The measurement error vector $\underline{\varepsilon}$ is assumed to be, zero mean, normally distributed, with known covariance,

$$
E(\underline{\varepsilon})=0
$$




$$
E\left(\underline{\varepsilon} \underline{\varepsilon}^{T}\right)=R
$$

where $E$ denotes the expected value, and $R$ is the measurement covariance matrix. It is also assumed that the measurement errors are uncorrelated, so that $R$ is a diagonal matrix. Therefore $[R]_{i j}=\delta_{i j} \sigma_{j}^{2}$, where $\sigma_{j}$ is the standard deviation of the $j$ th measurement and $\delta_{i j}$ is the Kronecker delta. The state estimates are said to be unbiased if and only if (3-2) and (3-3) are satisfied.

The optimal state estimate vector $\underline{x}$ may be determined by minimizing the sum of weighted squares of residuals:

$$
\min _{x} F(\underline{x})=[\underline{z}-\underline{h} \quad(\underline{x})]^{T} R^{-1}[\underline{z}-\underline{h} \quad(\underline{x})]
$$

Equation (3-4) is linearized using a Taylor series expansion, retaining the first two terms and ignoring higher order terms. This leads to a linear weighted least squares problem having the solution:

$$
\Delta \underline{x}=\left(J^{T} R^{-1} J\right)^{-1} J^{T} R^{-1} \Delta \underline{z}
$$

where $J$ is the Jacobian of $\underline{h}(\underline{x})$.

Repeated linearization and solution of (3-5) then solves the non-linear problem via the Newton-Raphson approach. The dependence on the iteration index is implicitly assumed for $\Delta \underline{x}, J$ and $\Delta \underline{z}$, where the current state vector is updated at each iteration until a stopping criterion is reached. Further details of the WLS formulation are available in references [45-48]. 


\subsubsection{Uncertainty interval estimation via linear programming (UILP)}

Uncertainty intervals can be determined by the solution of a series of appropriately formulated optimization problems. Each measurement, with its associated uncertainty, can be represented by upper and lower limits. These constraint limits define the tolerances on the measurements (i.e. the range of values within which the true value of the measured quantity must lie). Minimizing a particular state variable of interest, subject to all the measurement inequality constraints, provides the lower bound on that state variable. Similarly, maximizing that state variable, again subject to all the measurement inequalities, provides the upper bound for that state. In mathematical form:

$$
\begin{gathered}
\min _{\underline{x}} x_{i} \\
\text { subject to } \underline{z}^{\prime} \leq \underline{h}(\underline{x}) \leq \underline{z}^{u}
\end{gathered}
$$

where $\underline{z}^{\prime}$ is the lower bound of the measurement vector and $\underline{z}^{\prime}$ is upper bound, with:

$$
\begin{aligned}
& \underline{z}^{\prime}=\underline{z}-\underline{\tau}^{-} \\
& \underline{z}^{\prime \prime}=\underline{z}+\underline{\tau}^{+}
\end{aligned}
$$

where $\underline{\tau}^{+}$and $\underline{\tau}^{-}$are the transducer tolerances. The tolerances describe the deterministic uncertainty of each measurement. They represent the overall accuracy of the meter and can usually be provided by the manufacturer. Different values for the elements of positive and negative tolerances are permissible so that a transducer can be specified to have asymmetric accuracy if required (e.g. an accuracy of $-3 \%$ to $+5 \%$ of the nominal value). However, without loss of generality, we will usually assume that $\underline{\tau}^{+}=\underline{\tau}^{-}=\underline{\tau}$, giving a symmetric tolerance around the nominal value. 
Equation (3-6) defines a non-linear constrained optimization problem, which can be solved directly by a suitable non-linear programming algorithm such as Sequential Quadratic Programming (SQP) [49]. However, it is known that power system models are amenable to solution using the Newton-Raphson approach. Consequently, an alternative approach is to linearize equation (3-6) about a suitable point $\underline{\hat{x}}$ (which in this case can be provided by the WLS estimate) and then a series of linear programmes are solved to obtain updates $d x_{i}$ to the uncertainty bounds on the state variables. For example, the incremental change to the lower bound for the $i^{\text {th }}$ state can be computed by solving the following LP problem:

$$
\text { subject to } \quad \Delta \underline{z}^{\prime} \leq J \underline{\min _{\Delta}} \leq \Delta \underline{x}_{i}
$$

where $d x_{i}$ is the $i^{\text {th }}$ element of $\Delta \underline{x}$. Similarly, the incremental change to the upper bound on the $i^{\text {th }}$ state can be found by solving the LP problem:

$$
\text { subject to } \quad \Delta \underline{z}^{\prime} \leq J \underline{\max _{\Delta \underline{x}}} \leq \Delta \underline{z}^{u}
$$

where $J$ is the Jacobian of $h(\underline{x})$ evaluated at $\underline{\hat{x}}$, and $\Delta \underline{z}^{\prime}$ and $\Delta \underline{z}^{\prime}$ are vectors of the incremental changes to measurement lower and upper bounds respectively, computed in the following form:

$$
\begin{aligned}
& \Delta \underline{z}^{\prime}=\underline{z}^{\prime}-h(\underline{\hat{x}}) \\
& \Delta \underline{z}^{\prime \prime}=\underline{z}^{\prime}-h(\underline{\hat{x}})
\end{aligned}
$$


Therefore by performing $2 n$ linear programming solutions, all the elements of the vectors $d \underline{x}^{+}$and $d \underline{x}^{-}$can be calculated. Once $d \underline{x}^{+}$and $d \underline{x}^{-}$are known, the bounds on $\underline{\hat{x}}$ are simply found as:

$$
\begin{aligned}
& \underline{x}^{+}=\underline{\hat{x}}+d \underline{x}^{+} \\
& \underline{x}^{-}=\underline{\hat{x}}+d \underline{x}^{-}
\end{aligned}
$$

where $\underline{\hat{x}}$ is the point estimate obtained by WLS.

The complexity of the of the optimization problems in (3.9) and (3.10) is of the order $O\left(\|\underline{x}-\underline{\hat{x}}\|^{2}\right),[50,51]$. The computational burden of the process arises from the need to perform two LP solutions for every uncertainty interval sought. Nevertheless, with the measurement redundancy level available in power systems, the computational time is reasonable using modern hardware and software. For large networks it is possible that the dual LP formulation could be applied to reduce the execution time [52-54].

\subsubsection{Uncertainty interval calculation for other quantities:}

In addition to solving for the uncertainty ranges of the state variables (voltage magnitudes and phase angles), it is possible to compute the uncertainty range of other estimated quantities (such as power flows and injections), whether these quantities are measured or not. For example, with a change in the objective functions of (9) and (10), the incremental change in the lower bound of the $i^{\text {th }}$ measurement could be found by solving the following LP problem: 


$$
\min _{\Delta \underline{x}} \underline{j}_{i} \Delta \underline{x}
$$

subject to $\quad \Delta \underline{z}^{\prime} \leq J \Delta \underline{x} \leq \Delta \underline{z}^{\prime \prime}$

where $\underline{j}_{i}$ is the $i^{\text {th }}$ row of the Jacobian corresponding to the $i^{\text {th }}$ measurement. The solution of (3-15) is a set of incremental changes for all system state variables. Evaluating the objective function at this solution, i.e. $\underline{j}_{i}[\Delta \underline{\underline{x}}]_{\min }$, provides the incremental change in the lower bound for the $i^{\text {th }}$ measurement.

Similarly, the incremental change in the upper bound of the $i^{\text {th }}$ measurement is constructed from:

$$
\begin{gathered}
\max _{\Delta \underline{x}} \underline{j} \Delta \underline{x} \\
\text { subject to } \quad \Delta \underline{z}^{\prime} \leq J \Delta \underline{x} \leq \Delta \underline{z}^{u}
\end{gathered}
$$

Ultimately, the uncertainty bounds are computed as:

$$
\begin{aligned}
& \underline{Z}_{i}{ }^{u}=\underline{Z}_{i}+\underline{j}_{i}[\Delta \underline{x}]_{\max } \\
& \underline{Z}_{i}^{\prime}=\underline{Z}_{i}+\underline{j}_{i}[\Delta \underline{x}]_{\min }
\end{aligned}
$$

Uncertainty estimation is a 'worst-case' analysis in the sense that the LPs are seeking the extreme limits of uncertainty for the quantity of interest. This property is illustrated in Figure 3.1, based on a simple example described in reference [50]. The sequence of LPs calculates the bounding polytope due to uncertainty, i.e. the interior diamond shape in the figure. Weighted least squares estimation, on the other hand, produces an 'average-case' or maximum likelihood estimate of $x$. 


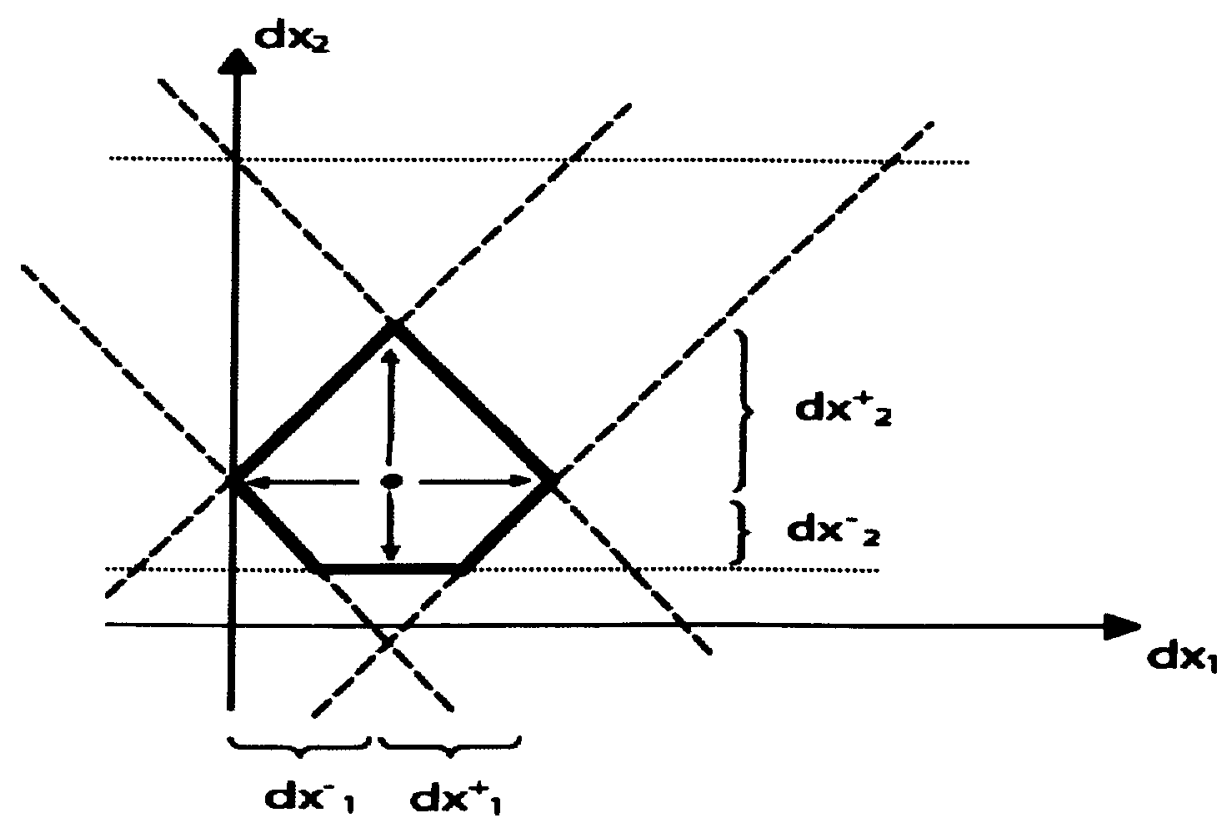

Figure 3.1 Two-dimensional example of LP uncertainty estimation

The geometry of the uncertainty estimation problem leads to the question of whether an infeasible problem might arise (i.e. no feasible polytope exists). This cannot occur if all the measurement uncertainties are correctly specified. However, if any gross measurement errors have not been eliminated, an infeasible problem is likely to arise. To avoid this in practice, it would be possible to perform gross error detection and elimination prior to the point estimation and uncertainty interval estimation procedures. In a later chapter, an extended robust formulation of the uncertainty problem will also be considered. 


\subsection{Comparison of Two Formulations}

This section compares two different methods for estimating the uncertainty interval in power system state estimation. The first is a non-linear formulation, solved by a Sequential Quadratic Programming (SQP) technique. The second relies upon two-step formulation using weighted least-squares analysis to compute 'point' state estimates and then linear programming to obtain the upper and lower bounds of the uncertainty intervals. A six-bus test system from [41] is used here as a test bed, to check the ability of both methods in accurately and efficiently estimating the uncertainty interval for power system state estimation problems.

The computation of all state variables will be shown to illustrate the concepts. However, for improved computational efficiency, only the variables of present interest to the power system operator would need to be computed.

The non-linear problems have been solved by the function fmincon incorporated in the MATLAB $^{\mathrm{TM}} 6.1$ optimization toolbox [49]. The function finds a solution by accumulating second order information regarding the KKT equations. This method is commonly referred to as Sequential Quadratic Programming (SQP). A detailed description of (SQP) will be shown in chapter 4. The linear programmes have been solved by the function linprog.

Table 4.1 presents results obtained by both methods, when applied to the 6-bus network. For the non-linear method, the upper and lower uncertainty bounds of the state variables 
are found using equations (3-1) to (3-3) with $\tau \equiv 3 \%$. The same tolerance was also used for the linear formulation. A WLS estimator was used to compute the (centre point) estimated states. Then, equations (3-4) to (3-9) are used to find the upper and lower bounds. It is apparent that both formulations provide almost identical estimates. Results of table 3.1 are illustrated in figure 3.2. We also notice that solution obtained by WLS is strictly bounded by the solution of SQP and WLS-LP.

Table 3.1 Estimated state variables of the 6-bus system with redundancy $\approx 2$.

\begin{tabular}{|c|c|c|c|c|c|c|c|c|}
\hline \multirow{2}{*}{ Bus \# } & \multicolumn{2}{|c|}{$\mathbf{L P}^{-}$(lower bound) } & \multicolumn{2}{c|}{$\mathbf{L P}^{+}{ }_{\text {(upper bound) }}$} & \multicolumn{2}{c|}{$\mathbf{S Q P}_{\text {(lower bound) }}$} & \multicolumn{2}{c|}{$\mathbf{S Q P}^{+}{ }_{\text {(upper bound) }}$} \\
\cline { 2 - 9 } & $|\mathbf{V}|(\mathrm{pu})$ & $\boldsymbol{\delta}(\mathrm{r} a d)$ & $|\mathbf{V}|(\mathrm{pu})$ & $\boldsymbol{\delta}(\mathrm{rad})$ & $|\mathbf{V}|(\mathrm{pu})$ & $\boldsymbol{\delta}(\mathrm{rad})$ & $|\mathbf{V}|(\mathrm{pu})$ & $\boldsymbol{\delta}(\mathrm{r} a d)$ \\
\hline $\mathbf{1}$ & 1.0175 & 0 & 1.0825 & 0 & 1.0175 & 0 & 1.0825 & 0 \\
\hline $\mathbf{2}$ & 1.0175 & -0.0912 & 1.0825 & -0.0388 & 1.0175 & -0.0905 & 1.0825 & -0.0380 \\
\hline $\mathbf{3}$ & 1.0375 & -0.1082 & 1.1025 & -0.0431 & 1.0375 & -0.1082 & 1.1025 & -0.0431 \\
\hline $\mathbf{4}$ & 0.9539 & -0.0888 & 1.0190 & -0.0571 & 0.9539 & -0.0890 & 1.0190 & -0.0572 \\
\hline $\mathbf{5}$ & 0.9471 & -0.1169 & 1.0122 & -0.0654 & 0.9471 & -0.1179 & 1.0122 & -0.0663 \\
\hline $\mathbf{6}$ & 0.9689 & -0.1367 & 1.0340 & -0.0717 & 0.9689 & -0.1367 & 1.0340 & -0.0717 \\
\hline
\end{tabular}




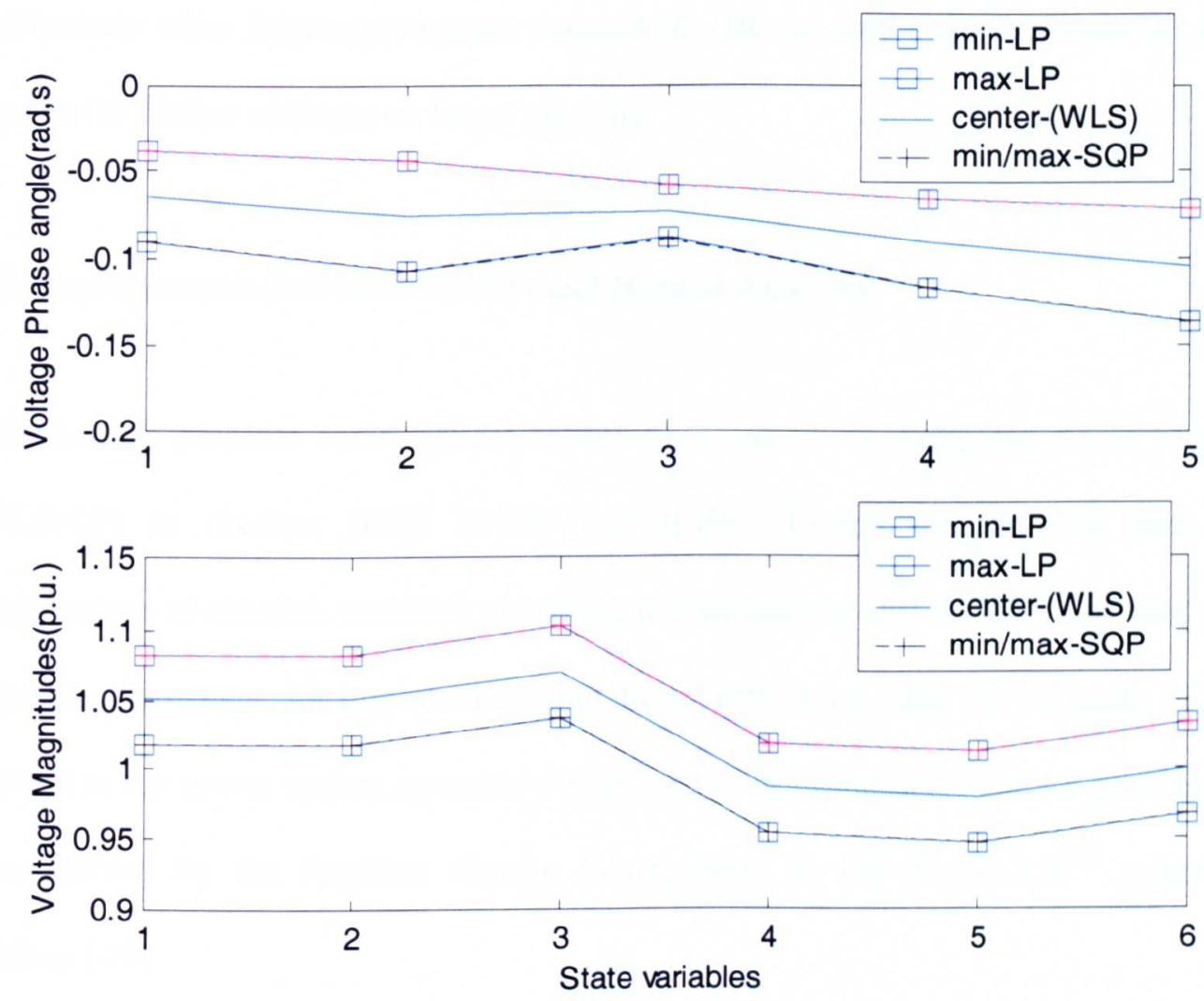

Figure 3.2 Estimated states for the six-bus system redundancy $\approx 2$

Table 3.2 Execution time of (WLS-LP) and (SQP)

\begin{tabular}{|c|c|c|}
\hline \multirow{2}{*}{ \# of measurements } & \multicolumn{2}{|c|}{ CPU time } \\
\cline { 2 - 3 } & Linear (WLS-LP) & Non-Linear(SQP) \\
\hline 23 (redundancy $\approx 2)$ & $0.201 \mathrm{sec}$ & $10.725 \mathrm{sec}$ \\
\hline 67 (Full) & $0.270 \mathrm{sec}$ & $15.743 \mathrm{sec}$ \\
\hline
\end{tabular}

Table 3.2 shows execution time for both methods with different redundancy levels (CPU:

Pentium 4, $1.7 \mathrm{GHZ}$ ). A redundancy $\approx 2$ and full set of measurements are used (all possible constraints: 12 injection equations, 44 flow equations and 11 state equations). Clearly, the linear (WLS-LP) outperforms the non-linear method, in these tests. The 
linear (WLS-LP) algorithm seems more suitable for estimating uncertainty interval, particularly when larger systems are considered. Hence, in the next section (WLS-LP) is chosen for further analyses on larger systems.

\subsection{Implementation of Case Studies and Results Analysis}

This section presents some typical results obtained by applying the linear algorithms (WLS-LP) to six-bus, IEEE 30-bus and IEEE 118-bus test network data. The computation of all state variables and some measurements will be shown to illustrate the concepts. However, for improved computational efficiency, only the variables of present interest to the power system operator would need to be computed. The LP problems have been solved by the function linprog incorporated in the MATLAB $^{\mathrm{TM}}$ optimization toolbox [49]. 


\subsubsection{Six Bus System}

3.5.1.1 Confidence Bounds Analysis with (UILP)

Table 3.3 Estimated state variables and uncertainty bounds for the six-bus network

\begin{tabular}{|c|c|c|c|c|c|c|c|c|}
\hline \multirow{2}{*}{ Bus \# } & \multicolumn{2}{|c|}{ Simulated } & \multicolumn{2}{c|}{$\mathbf{L P}_{\text {(lower bound) }}$} & \multicolumn{2}{c|}{ WLS $_{\text {(centre point) }}$} & \multicolumn{2}{c|}{$\mathbf{L P}^{+}$(upper bound) } \\
\cline { 2 - 9 } & $|\mathbf{V}|(\mathrm{pu})$ & $\boldsymbol{\delta}(\mathrm{rad})$ & $|\mathrm{V}|(\mathrm{pu})$ & $\boldsymbol{\delta}(\mathrm{rad})$ & $|\mathrm{V}|(\mathrm{pu})$ & $\boldsymbol{\delta}(\mathrm{rad})$ & $|\mathrm{V}|(\mathrm{pu})$ & $\boldsymbol{\delta}(\mathrm{rad})$ \\
\hline $\mathbf{1}$ & 1.0500 & 0 & 1.0417 & 0 & 1.0738 & 0 & 1.1018 & 0 \\
\hline $\mathbf{2}$ & 1.0500 & -0.0650 & 1.0265 & -0.0908 & 1.0678 & -0.0612 & 1.0908 & -0.0440 \\
\hline $\mathbf{3}$ & 1.0700 & -0.0756 & 1.0248 & -0.1027 & 1.0606 & -0.0683 & 1.0891 & -0.0560 \\
\hline $\mathbf{4}$ & 0.9864 & -0.0729 & 0.9678 & -0.0865 & 1.0058 & -0.0693 & 1.0321 & -0.0541 \\
\hline $\mathbf{5}$ & 0.9797 & -0.0912 & 0.9395 & -0.0891 & 0.9614 & -0.0845 & 1.0038 & -0.0664 \\
\hline $\mathbf{6}$ & 1.0014 & -0.1042 & 0.9768 & -0.0900 & 1.0005 & -0.0840 & 1.0411 & -0.0257 \\
\hline
\end{tabular}

Table 3.3 shows a comparison of simulated (from a load flow solution) and estimated states for the six-bus network. The measurement uncertainty has been represented as a uniform distribution over the interval $[-3 \%, 3 \%]$ of the nominal value of the measurements. A WLS estimator was used to compute the (centre point) estimated states. Discrepancies between the simulated and the estimated centre point are fairly large, due to the significant noise level (i.e. $\pm 3 \%$, uniformly distributed). The upper and lower uncertainty bounds of the state variables are found using equations (3-9) to (3-12), with $\tau \equiv 3 \%$. It is apparent that the centre point estimates are within the upper and lower uncertainty bounds, in this case as illustrated by figure 3.3 . 

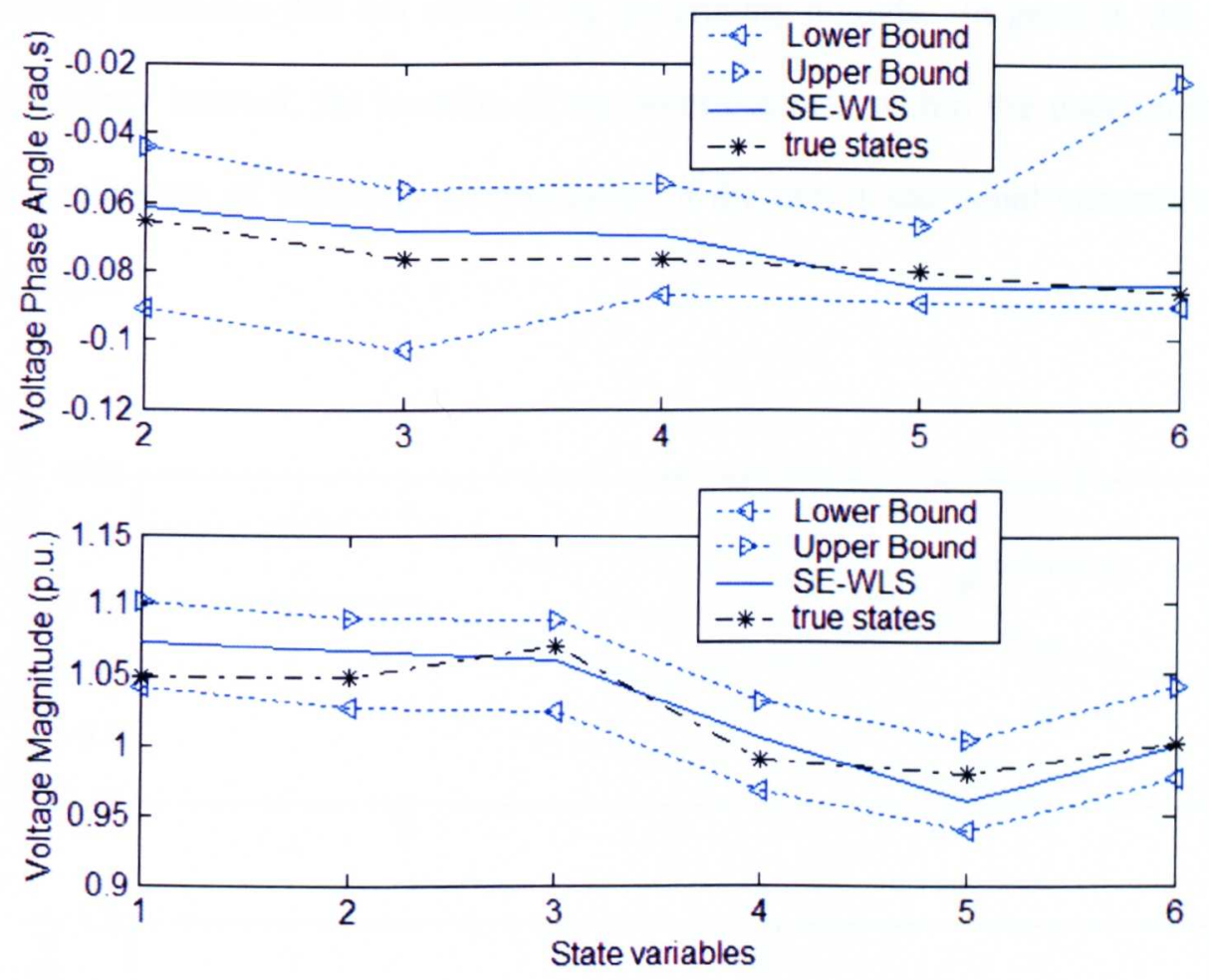

Figure 3.3 Estimated states and uncertainty bounds for the six-bus system

Further tests were undertaken to examine the effects of parameter uncertainty in the power network parameters. An error of $+3 \%$ was introduced into the resistance, inductance and capacitance parameters of the most heavily loaded line (connecting bus 1 and bus 4). Figure 3.4 shows the estimated states with their bounds (which are designated by '+' signs).

In the phase angle results, it is interesting to note that a 'crossing' occurs. The estimated state $\boldsymbol{\delta}_{\mathbf{5}}$ does not lie within its calculated bounds. This crossing suggests that the estimated centre point is inaccurate. The WLS process assumes normally distributed errors (where errors of any magnitude are considered to be possible) and can therefore 
produce estimates that fall outside the uncertainty bounds. In general, the width of the uncertainty interval, the location of the point estimate within the uncertainty range, and the occurrence of 'crossing' are examples of the useful additional information generated by UILP.
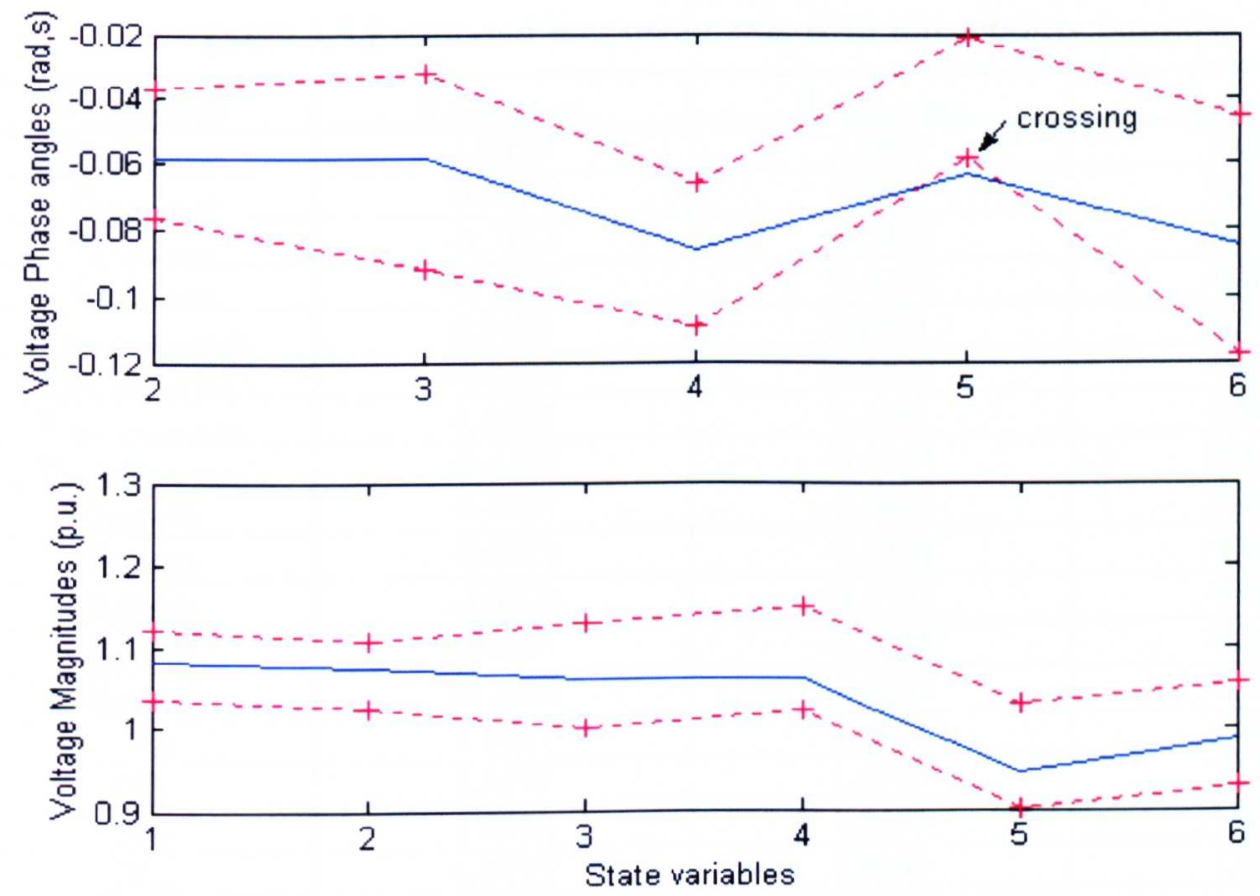

Figure 3.4 Estimated states and uncertainty bounds for the six-bus network

The execution times of the of the proposed method for the six-bus network are given in table 3.4; which provides the CPU time for test cases with both a full measurement set and a subset sets of measurements (redundancy $\approx 2.0$ to 2.5 ).

Table 3.4 CPU and execution time of six-bus network

\begin{tabular}{|c|c|c|}
\hline Test system & \# of measurements (\& constraints) & CPU time \\
\hline \hline 6-bus & $28($ redundancy $\approx 2)$ & $0.24 \mathrm{sec}$ \\
\hline 6-bus & $67($ Full $)$ & $0.38 \mathrm{sec}$ \\
\hline
\end{tabular}




\subsubsection{Confidence Bounds Analysis of other Quantities}

Equations (3-15) to (3-18) may be used to find uncertainty bounds for measured and unmeasured quantities, in addition to the state variables. In Table 3.5, selected estimated measurements are presented with their bounds.

Table 3.5 Estimated measurements with uncertainty bounds

\begin{tabular}{|c|c|c|c|}
\hline Type (pu) & $\mathbf{Z}_{\text {(lower bound) }}$ & $\mathbf{Z}_{\text {(centre point) }}$ & $\mathbf{Z}_{\text {(upper bound) }}$ \\
\hline $\mathbf{P}_{1 \text { (iniection) }}$ & 1.0439 & 1.0824 & 1.0835 \\
\hline $\mathbf{P}_{2 \text { (iniection) }}$ & 0.4536 & 0.4843 & 0.5187 \\
\hline$P_{5 \text { (iniertion) }}$ & -0.7283 & -0.6851 & -0.6632 \\
\hline $\mathbf{Q}_{1 \text { (iniection) }}$ & -0.9623 & -0.9295 & -0.8972 \\
\hline $\mathbf{P}_{\text {bine (busl to hus 2) }}$ & 0.2924 & 0.2924 & 0.3140 \\
\hline $\mathbf{P}_{\text {line (bur2 no busl) }}$ & -0.2990 & -0.2822 & -0.2765 \\
\hline$P_{\text {line (bus1 io bust) }}$ & 0.4262 & 0.4441 & 0.4683 \\
\hline$P_{\text {line (bus to busl) }}$ & -0.4581 & -0.4334 & -0.4160 \\
\hline$\delta_{2}(\mathrm{rad})$ & -0.0861 & -0.0662 & -0.0538 \\
\hline$\delta_{3}(\mathrm{rad})$ & -0.0827 & -0.0638 & -0.0469 \\
\hline$\delta_{4}(\mathrm{rad})$ & -0.0809 & -0.0725 & -0.0609 \\
\hline$\delta_{5}(\mathrm{rad})$ & -0.0801 & -0.0799 & -0.0622 \\
\hline$\delta_{6}(\mathrm{rad})$ & -0.1094 & -0.0936 & -0.0704 \\
\hline$|\mathbf{V}|_{1}$ & 1.0481 & 1.0672 & 1.0886 \\
\hline$|\mathbf{V}|_{2}$ & 1.0397 & 1.0747 & 1.1048 \\
\hline$|\mathbf{V}|_{3}$ & 1.0140 & 1.0553 & 1.0790 \\
\hline$|\mathbf{V}|_{4}$ & 0.9725 & 1.0085 & 1.0376 \\
\hline$|\mathrm{V}|_{\mathbf{5}}$ & 0.9569 & 0.9702 & 1.0168 \\
\hline$\left.\mathrm{V}\right|_{6}$ & 0.9642 & 0.9943 & 1.0293 \\
\hline
\end{tabular}


Figure 3.5 shows all possible estimated measurements, with bounds for the 6-bus network. The results are sorted in descending order for clarity.

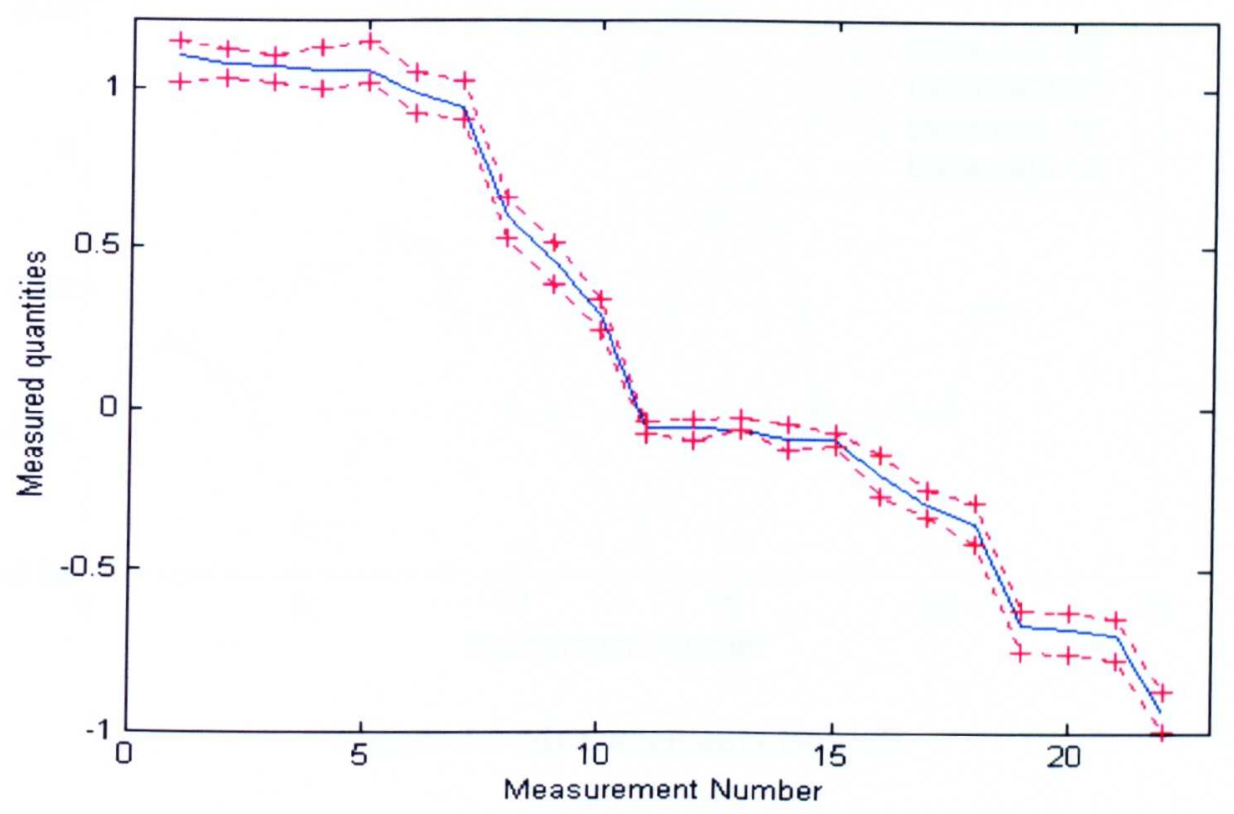

Figure 3.5 the estimated measurements with uncertainty bounds for six-bus system

The upper and lower bounds of all measurement changes from equations 3-11 and 3-12 are plotted in figure 3.6 along with the incremental changes of measurement obtained from the solution of the optimization problems of equations 3-15 and 3-16. Interestingly, the estimated incremental bounds of all measurements, (i.e. solution from the optimization problem), either lie within or on the allowed bounds. 


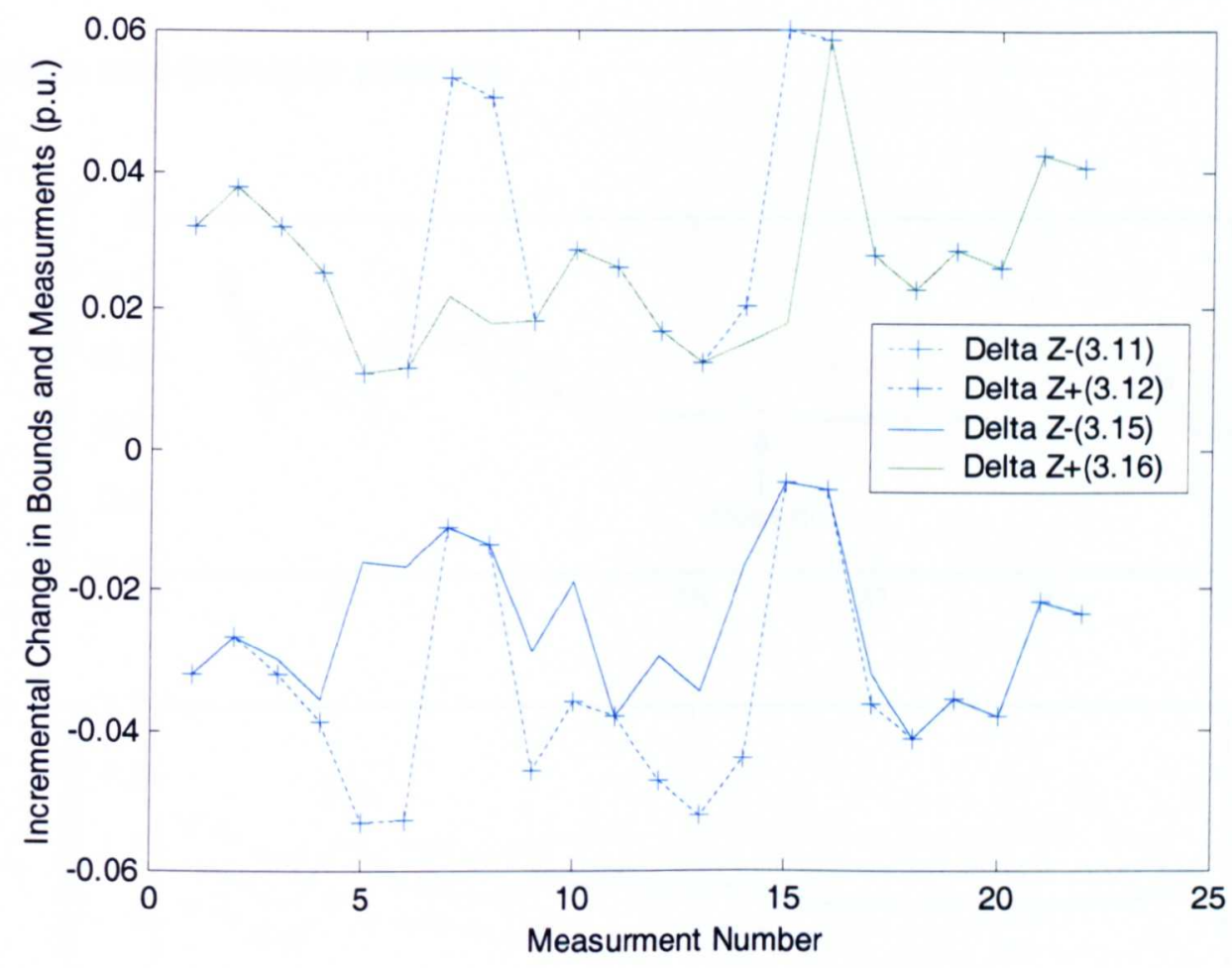

Figure 3.6 Measurements Bounds

\subsubsection{IEEE 30-bus test System}

Tests were also conducted on the IEEE 30-bus network. In this example, $+10 \%$ parameter errors are introduced for the most heavily loaded line. With $\tau \equiv 6 \%$, the estimated states and bounds are shown in table 3.6 and Figure 3.7. A 'crossing' is again apparent. The state $\boldsymbol{\delta}_{17}$ does not lie within its bounds.

In the tests presented here and in further tests the Newton Raphson process was found to perform reliably, with convergence occurring within 3 to 4 iterations. This is consistent 
with the behaviour of the Newton Raphson process in solving other types of power system state estimation problems.
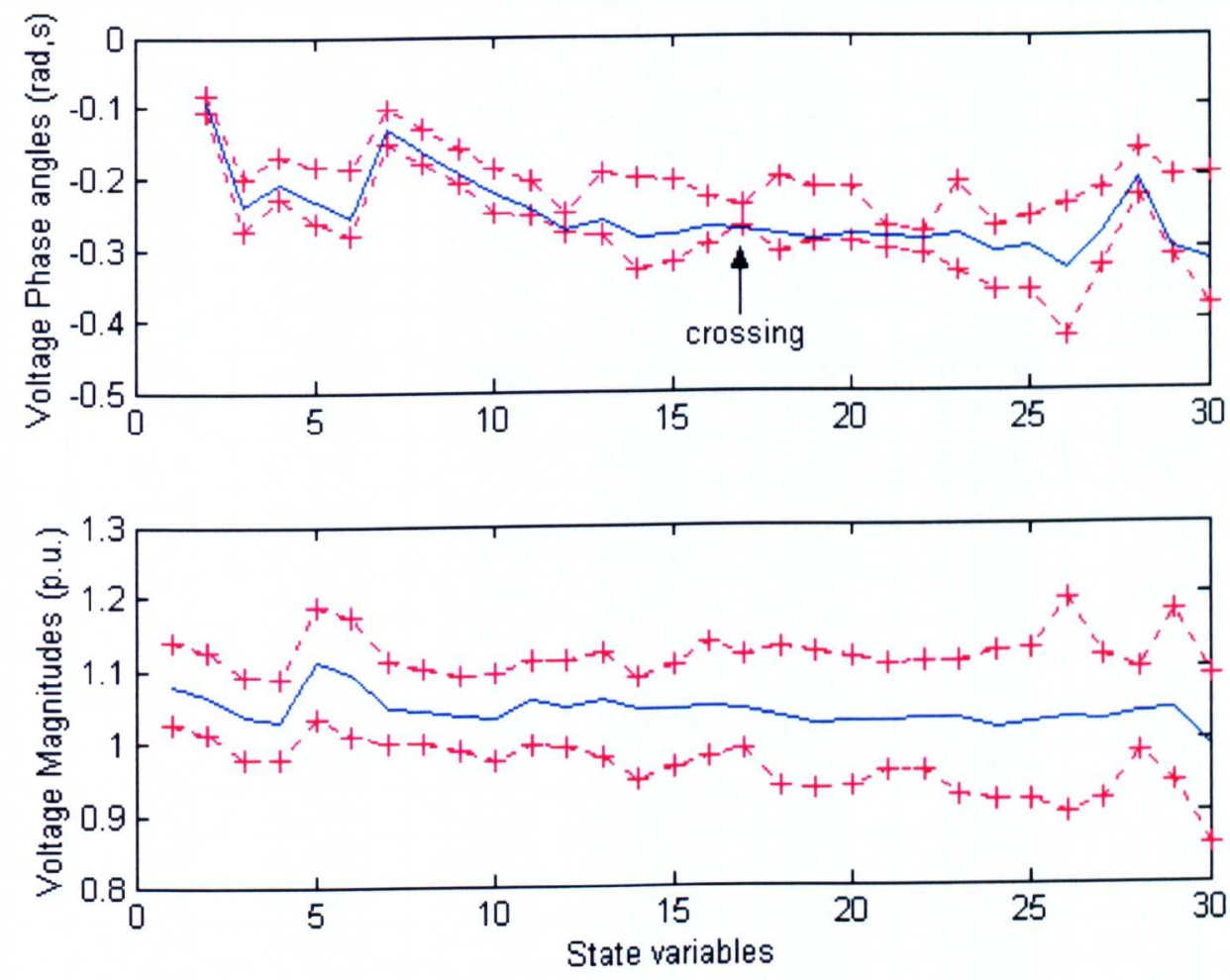

Figure 3.7 Estimated states and uncertainty bounds for the IEEE 30-bus network 
Table 3.6 Estimated state variables and uncertainty bounds for the IEEE 30-bus network.

\begin{tabular}{|c|c|c|c|c|c|c|c|c|}
\hline \multirow[b]{2}{*}{ Bus \# } & \multicolumn{2}{|c|}{ True states } & \multicolumn{2}{|c|}{$\mathbf{L P}_{\text {(lower bound) }}^{-}$} & \multicolumn{2}{|c|}{ WLS $_{\text {(centre point) }}$} & \multicolumn{2}{|c|}{$\mathbf{L P}_{\text {(upper bound) }}^{+}$} \\
\hline & $|\mathbf{v}|(\mathrm{pu})$ & $\delta(\mathrm{rad})$ & $|\mathrm{v}|(\mathrm{pu})$ & $\delta(\mathrm{rad})$ & $|\mathrm{V}|(\mathrm{pu})$ & $\delta(\mathrm{rad})$ & $|\mathbf{v}|(\mathrm{pu})$ & $\delta(\mathrm{rad})$ \\
\hline 1 & 1.0600 & 0 & 1.0261 & 0 & 1.0783 & 0 & 1.1410 & 0 \\
\hline 2 & 1.0430 & -0.0932 & 1.0124 & -0.1053 & 1.0641 & -0.0926 & 1.1265 & -0.0817 \\
\hline 3 & 1.0269 & -0.1328 & 0.9994 & -0.1494 & 1.0472 & -0.1305 & 1.1129 & -0.1010 \\
\hline 4 & 1.0194 & -0.1635 & 0.9998 & -0.1782 & 1.0414 & -0.1611 & 1.1016 & -0.1293 \\
\hline 5 & 1.0100 & -0.2467 & 0.9785 & -0.2738 & 1.0361 & -0.2378 & 1.0903 & -0.2011 \\
\hline 6 & 1.0138 & -0.1937 & 0.9884 & -0.2077 & 1.0360 & -0.1910 & 1.0909 & -0.1581 \\
\hline 7 & 1.0045 & -0.2247 & 0.9739 & -0.2495 & 1.0311 & -0.2210 & 1.0941 & -0.1854 \\
\hline 8 & 1.0100 & -0.2057 & 0.9769 & -0.2270 & 1.0289 & -0.2051 & 1.0879 & -0.1685 \\
\hline 9 & 1.0364 & -0.2507 & 0.9960 & -0.2500 & 1.0566 & -0.2400 & 1.1111 & -0.2041 \\
\hline 10 & 1.0256 & -0.2804 & 0.9899 & -0.2777 & 1.0459 & -0.2737 & 1.1119 & -0.2481 \\
\hline 11 & 1.0820 & -0.2 & 1.0322 & -0.2627 & 1.1135 & -0.2292 & 1.1873 & -0.1810 \\
\hline 12 & 1.0340 & -0.2681 & 0.9771 & -0.2806 & 1.0553 & -0.2577 & 1.1220 & -0.1922 \\
\hline 13 & 1.0710 & -0.2681 & 1.0076 & -0.2787 & 1.0943 & -0.2563 & 1.1743 & -0.1862 \\
\hline 14 & 1.0191 & -0.2841 & 0.9466 & -0.3297 & 1.0415 & -0.2825 & 1.0883 & -0.2011 \\
\hline 15 & 1.0148 & -0.2 & 0.9647 & -0.3191 & 1.0437 & -0.2785 & 1.1061 & -0.2016 \\
\hline 16 & 1.0228 & -0. & 0.9762 & -0.2928 & 1.0449 & -0.2691 & 1.1360 & -0.2261 \\
\hline 17 & 1.0196 & -0 . & 0.9886 & 679 & 1.0443 & -0.2734 & 1.1183 & -0.2383 \\
\hline 18 & 1.0062 & & 0.9352 & -0.3039 & 1.0345 & -0.2809 & 1.1302 & -0.1994 \\
\hline 19 & 1.0043 & -0.2994 & 0.9323 & -0.2893 & 1.0220 & -0.2851 & 1.1211 & -0.2141 \\
\hline 20 & 1.0089 & -0.2957 & 0.9373 & -0.2914 & 1.0270 & -0.2791 & 1.1172 & -0.2150 \\
\hline 21 & 1.0125 & -0.2 & 0.9564 & -0.3013 & 1.0264 & 841 & 1.1051 & -0.2707 \\
\hline 22 & 1.0128 & -0 . & 0.9573 & -0.3062 & 1.0285 & 860 & 1.1102 & -0.2755 \\
\hline 23 & 1.0042 & -0. & 0.9224 & -0.3 & 1.0289 & 300 & 1.1075 & -0.2059 \\
\hline 24 & 0.9987 & -0.2945 & 0.9156 & -0.3609 & 1.0168 & -0.3049 & 1.1226 & -0.2692 \\
\hline 25 & 0.9914 & -0.2855 & 0.9161 & -0.3593 & 1.0213 & -0.2963 & 1.1273 & -0.2554 \\
\hline 26 & 0.9732 & -0.2932 & 0.8981 & -0.4242 & 1.0302 & -0.3264 & 1.1943 & -0.2393 \\
\hline 27 & 0.9956 & -0.2752 & 0.9172 & -0.3246 & 1.0269 & & 1.1145 & -0.2154 \\
\hline 28 & 1.0099 & & 0.9801 & -0.2289 & 1.0357 & & 1.0993 & -0.1619 \\
\hline 29 & 0.9752 & -0.2979 & 0.9386 & -0.3091 & 1.0384 & -0.3005 & 1.1784 & -0.1997 \\
\hline 30 & 0.9633 & -0.3142 & 0.8544 & -0.3818 & 0.9892 & -0.3184 & 1.0882 & -0.1967 \\
\hline
\end{tabular}

The execution time of the of the proposed methods on the IEEE 30-bus network is shown in table 3.7 which provide the CPU time for both full set of measurements (redundancy $\approx$ 3.98 ) and, a reduced set of measurements (redundancy $\approx 2.0$ to 2.5 ). 
Table 3.7 CPU and execution time of IEEE 30-bus system

\begin{tabular}{|c|c|c|}
\hline Test system & \# of measurements $(\&$ constraints $)$ & CPU time \\
\hline \hline IEEE 30 -bus & 150 (redundancy $\approx 2.5)$ & $1.9336 \mathrm{~min}$ \\
\hline IEEE 30-bus & 283 (Full) & $4.0535 \mathrm{~min}$ \\
\hline
\end{tabular}

\subsubsection{IEEE 118-bus test System}

Tests were also conducted on the IEEE 118-bus network. In this example, $\tau \equiv 10 \%$, all estimated states and bounds are shown in table 3.8. Figure 3.8 illustrates the estimated phase angles from bus 1 to 6 of the IEEE 118-bus network. Figure 3.9 shows the estimated voltage magnitudes with their bounds from bus 77 to 82 .

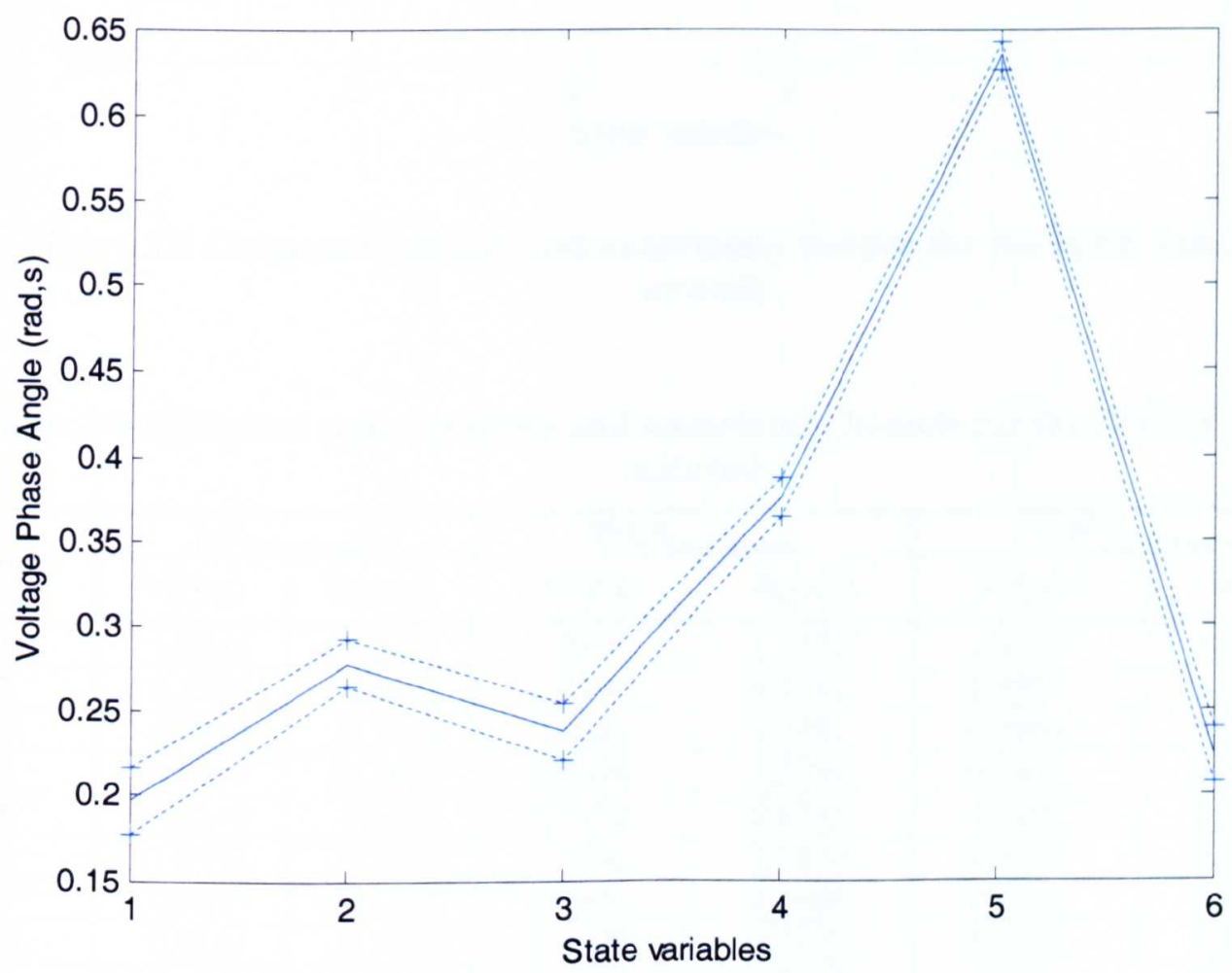

Figure 3.8 Estimated phase angles and uncertainty bounds for the IEEE 118-bus network 


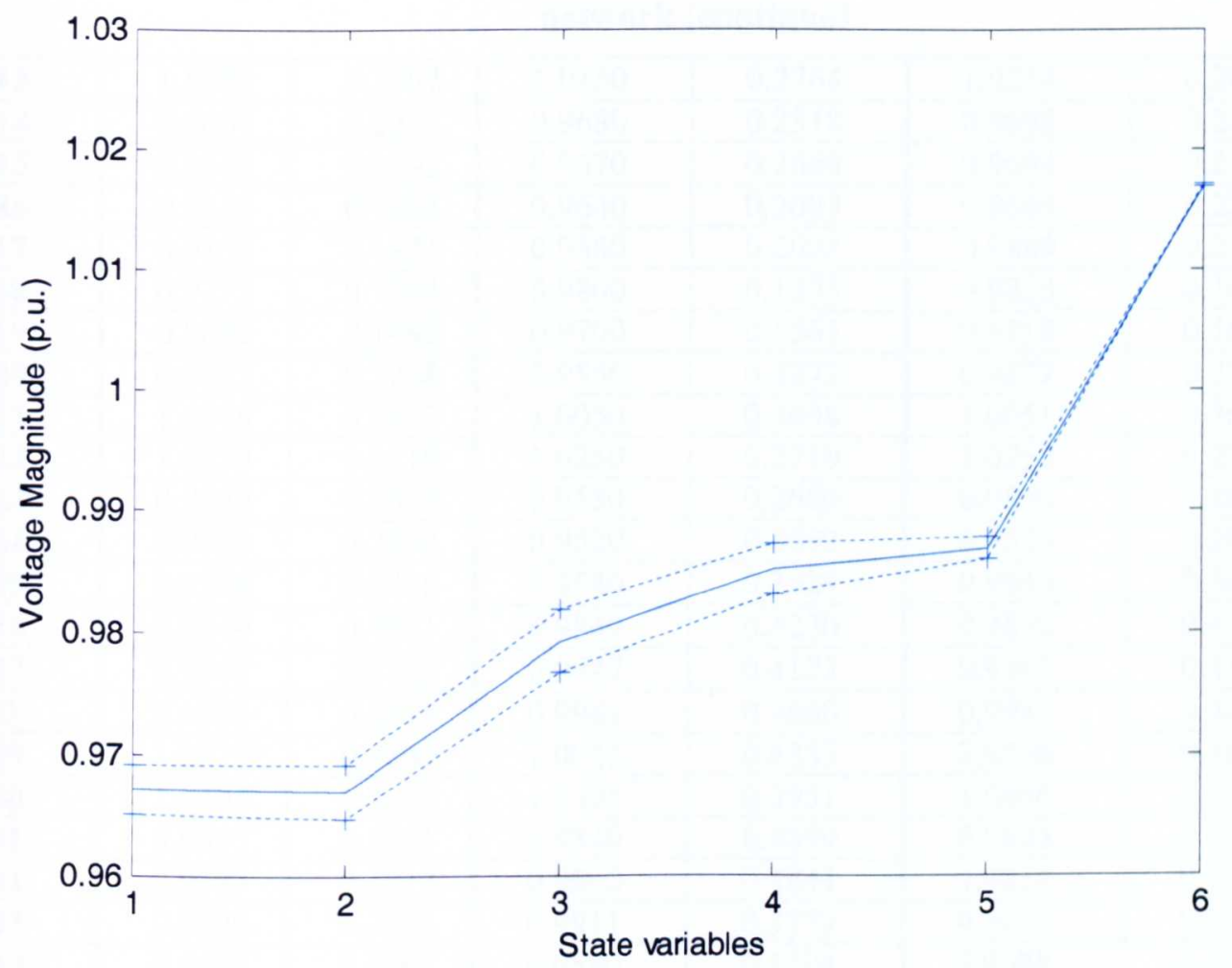

Figure 3.9 Estimated voltages and uncertainty bounds for the IEEE 118-bus network

Table 3.8 Estimated state variables and uncertainty bounds for the IEEE 118-bus network

\begin{tabular}{|c||c|c||c|c||c|c|}
\hline \multirow{2}{*}{ Bus \# } & \multicolumn{2}{|c|}{$\mathbf{L P}_{\text {(lower bound) }}^{-}$} & \multicolumn{2}{c|}{$\mathbf{W L S}_{\text {(centre point) }}$} & \multicolumn{2}{c|}{$\mathbf{L P}^{+}{ }_{\text {(upper bound) }}$} \\
\cline { 2 - 7 } & $|\mathbf{V}|(\mathrm{pu})$ & $\boldsymbol{\delta}(\mathrm{r}$ ad $)$ & $|\mathbf{V}|(\mathrm{pu})$ & $\boldsymbol{\delta}(\mathrm{rad})$ & $|\mathbf{V}|(\mathrm{pu})$ & $\boldsymbol{\delta}(\mathrm{r}$ ad $)$ \\
\hline $\mathbf{1}$ & 1.0346 & 0.1777 & 1.0350 & 0.1974 & 1.0355 & 0.2172 \\
\hline $\mathbf{2}$ & 0.9546 & 0.2641 & 0.9550 & 0.2782 & 0.9554 & 0.2922 \\
\hline $\mathbf{3}$ & 0.9974 & 0.2216 & 0.9980 & 0.2381 & 0.9986 & 0.2546 \\
\hline $\mathbf{4}$ & 0.9900 & 0.3635 & 0.9900 & 0.3754 & 0.9900 & 0.3872 \\
\hline $\mathbf{5}$ & 1.0140 & 0.6260 & 1.0150 & 0.6343 & 1.0159 & 0.6426 \\
\hline $\mathbf{6}$ & 1.0472 & 0.2079 & 1.0500 & 0.2240 & 1.0527 & 0.2401 \\
\hline $\mathbf{7}$ & 0.9893 & 0.1933 & 0.9900 & 0.2066 & 0.9907 & 0.2199 \\
\hline $\mathbf{8}$ & 0.9693 & 0.2002 & 0.9700 & 0.2120 & 0.9707 & 0.2238 \\
\hline $\mathbf{9}$ & 0.9725 & 0.1908 & 0.9730 & 0.2037 & 0.9735 & 0.2165 \\
\hline $\mathbf{1 0}$ & 0.9625 & 0.3658 & 0.9630 & 0.3711 & 0.9635 & 0.3764 \\
\hline $\mathbf{1 1}$ & 0.9907 & 0.4884 & 0.9920 & 0.4946 & 0.9933 & 0.5008 \\
\hline $\mathbf{1 2}$ & 1.0482 & 0.5161 & 1.0500 & 0.5253 & 1.0518 & 0.5344 \\
\hline
\end{tabular}


Table 3.8 Estimated state variables and uncertainty bounds for the IEEE 118-bus network (continue)

\begin{tabular}{|c|c|c|c|c|c|c|}
\hline 13 & 1.0085 & 0.2697 & 1.0150 & 0.2764 & 1.0214 & 0.2831 \\
\hline 14 & 0.9661 & 0.2231 & 0.9680 & 0.2318 & 0.9698 & 0.2404 \\
\hline 15 & 0.9645 & 0.2592 & 0.9670 & 0.2669 & 0.9694 & 0.2745 \\
\hline 16 & 0.9616 & 0.1985 & 0.9640 & 0.2093 & 0.9664 & 0.2202 \\
\hline 17 & 0.9832 & 0.1921 & 0.9860 & 0.2022 & 0.9889 & 0.2122 \\
\hline 18 & 0.9775 & 0.1284 & 0.9800 & 0.1375 & 0.9825 & 0.1466 \\
\hline 19 & 0.9682 & 0.1498 & 0.9700 & 0.1563 & 0.9718 & 0.1629 \\
\hline 20 & 0.9827 & 0.3268 & 0.9850 & 0.3273 & 0.9872 & 0.3279 \\
\hline 21 & 1.0048 & 0.3697 & 1.0050 & 0.3698 & 1.0051 & 0.3699 \\
\hline 22 & 1.0250 & 0.2710 & 1.0250 & 0.2710 & 1.0250 & 0.2711 \\
\hline 23 & 0.9550 & 0.2660 & 0.9550 & 0.2660 & 0.9550 & 0.2660 \\
\hline 24 & 0.9520 & 0.2692 & 0.9520 & 0.2692 & 0.9520 & 0.2692 \\
\hline 25 & 0.9540 & 0.3416 & 0.9540 & 0.3429 & 0.9540 & 0.3441 \\
\hline 26 & 0.9849 & 0.4221 & 0.9849 & 0.4230 & 0.9850 & 0.4239 \\
\hline 27 & 0.9947 & 0.4122 & 0.9947 & 0.4123 & 0.9947 & 0.4124 \\
\hline 28 & 0.9981 & 0.4824 & 0.9981 & 0.4860 & 0.9981 & 0.4896 \\
\hline 29 & 1.0015 & 0.4801 & 1.0045 & 0.4835 & 1.0076 & 0.4868 \\
\hline 30 & 1.0495 & 0.3922 & 1.0495 & 0.3951 & 1.0496 & 0.3979 \\
\hline 31 & 0.9835 & 0.3651 & 0.9840 & 0.3699 & 0.9845 & 0.3747 \\
\hline 32 & 0.9787 & 0.3817 & 0.9800 & 0.3844 & 0.9814 & 0.3871 \\
\hline 33 & 0.9906 & 0.3753 & 0.9911 & 0.3779 & 0.9916 & 0.3805 \\
\hline 34 & 0.9573 & 0.3794 & 0.9580 & 0.3794 & 0.9588 & 0.3794 \\
\hline 35 & 0.9430 & 0.4645 & 0.9430 & 0.4649 & 0.9430 & 0.4653 \\
\hline 36 & 1.0059 & 0.5016 & 1.0061 & 0.5032 & 1.0062 & 0.5048 \\
\hline 37 & 1.0401 & 0.5655 & 1.0401 & 0.5655 & 1.0401 & 0.5655 \\
\hline 38 & 0.9850 & 0.5462 & 0.9850 & 0.5462 & 0.9850 & 0.5462 \\
\hline 39 & 1.0150 & 0.6909 & 1.0150 & 0.6909 & 1.0150 & 0.6909 \\
\hline 40 & 1.0050 & 0.5791 & 1.0050 & 0.5791 & 1.0050 & 0.5791 \\
\hline 41 & 0.9850 & 0.5795 & 0.9850 & 0.5795 & 0.9850 & 0.5795 \\
\hline 42 & 0.9800 & 0.5880 & 0.9800 & 0.5880 & 0.9800 & 0.5880 \\
\hline 43 & 0.9930 & 0.4699 & 0.9930 & 0.4699 & 0.9930 & 0.4699 \\
\hline 44 & 1.0101 & 0.4874 & 1.0101 & 0.4874 & 1.0101 & 0.4874 \\
\hline 45 & 1.0171 & 0.4245 & 1.0171 & 0.4245 & 1.0171 & 0.4245 \\
\hline 46 & 1.0011 & 0.3767 & 1.0011 & 0.3767 & 1.0011 & 0.3767 \\
\hline 47 & 0.9711 & 0.3574 & 0.9711 & 0.3574 & 0.9711 & 0.3574 \\
\hline 48 & 0.9651 & 0.3040 & 0.9651 & 0.3040 & 0.9651 & 0.3040 \\
\hline 49 & 0.9521 & 0.3138 & 0.9521 & 0.3138 & 0.9521 & 0.3138 \\
\hline 50 & 0.9731 & 0.3425 & 0.9731 & 0.3425 & 0.9731 & 0.3425 \\
\hline 51 & 0.9801 & 0.2597 & 0.9801 & 0.2597 & 0.9801 & 0.2597 \\
\hline 52 & 0.9751 & 0.2384 & 0.9751 & 0.2502 & 0.9751 & 0.2621 \\
\hline 53 & 0.9909 & 0.4696 & 0.9930 & 0.4744 & 0.9952 & 0.4791 \\
\hline 54 & 1.0352 & 0.1888 & 1.0356 & 0.2069 & 1.0360 & 0.2251 \\
\hline 55 & 0.9713 & 0.1955 & 0.9714 & 0.2133 & 0.9715 & 0.2310 \\
\hline 56 & 0.9662 & 0.2732 & 0.9663 & 0.2873 & 0.9664 & 0.3014 \\
\hline 57 & 0.9961 & 0.2137 & 0.9963 & 0.2303 & 0.9965 & 0.2469 \\
\hline
\end{tabular}


Table 3.8 Estimated state variables and uncertainty bounds for the IEEE 118-bus network (continue)

\begin{tabular}{|c|c|c|c|c|c|c|}
\hline 58 & 0.9891 & 0.4918 & 0.9893 & 0.5020 & 0.9895 & 0.5122 \\
\hline 59 & 1.0408 & 0.2175 & 1.0429 & 0.2333 & 1.0450 & 0.2491 \\
\hline 60 & 0.9839 & 0.1958 & 0.9841 & 0.2093 & 0.9842 & 0.2228 \\
\hline 61 & 0.9668 & 0.1970 & 0.9675 & 0.2116 & 0.9682 & 0.2262 \\
\hline 62 & 0.9831 & 0.2044 & 0.9836 & 0.2195 & 0.9841 & 0.2347 \\
\hline 63 & 0.9808 & 0.2414 & 0.9812 & 0.2531 & 0.9816 & 0.2648 \\
\hline 64 & 0.9865 & 0.2069 & 0.9868 & 0.2182 & 0.9870 & 0.2295 \\
\hline 65 & 0.9565 & 0.2369 & 0.9579 & 0.2453 & 0.9592 & 0.2537 \\
\hline 66 & 0.9568 & 0.2824 & 0.9586 & 0.2892 & 0.9604 & 0.2959 \\
\hline 67 & 0.9681 & 0.3680 & 0.9697 & 0.3739 & 0.9713 & 0.3798 \\
\hline 68 & 0.9991 & 0.2400 & 0.9998 & 0.2466 & 1.0004 & 0.2532 \\
\hline 69 & 0.9592 & 0.5236 & 0.9616 & 0.5236 & 0.9639 & 0.5236 \\
\hline 70 & 0.9606 & 0.2214 & 0.9632 & 0.2296 & 0.9658 & 0.2379 \\
\hline 71 & 1.0291 & 0.3270 & 1.0312 & 0.3380 & 1.0333 & 0.3490 \\
\hline 72 & 0.9663 & 0.1857 & 0.9673 & 0.1980 & 0.9683 & 0.2103 \\
\hline 73 & 0.9767 & 0.1924 & 0.9791 & 0.2025 & 0.9816 & 0.2126 \\
\hline 74 & 0.9804 & 0.2097 & 0.9830 & 0.2202 & 0.9856 & 0.2307 \\
\hline 75 & 1.0380 & 0.2956 & 1.0409 & 0.3051 & 1.0438 & 0.3145 \\
\hline 76 & 0.9651 & 0.1469 & 0.9671 & 0.1579 & 0.9690 & 0.1688 \\
\hline 77 & 0.9646 & 0.1213 & 0.9668 & 0.1294 & 0.9690 & 0.1375 \\
\hline 78 & 0.9767 & 0.1963 & 0.9792 & 0.2068 & 0.9818 & 0.2173 \\
\hline 79 & 0.9831 & 0.2418 & 0.9851 & 0.2480 & 0.9872 & 0.2541 \\
\hline 80 & 0.9859 & 0.2758 & 0.9868 & 0.2790 & 0.9877 & 0.2823 \\
\hline 81 & 1.0170 & 0.3654 & 1.0171 & 0.3656 & 1.0172 & 0.3658 \\
\hline 82 & 1.0206 & 0.3522 & 1.0206 & 0.3523 & 1.0207 & 0.3525 \\
\hline 83 & 1.0011 & 0.3343 & 1.0011 & 0.3343 & 1.0011 & 0.3343 \\
\hline 84 & 0.9669 & 0.2886 & 0.9669 & 0.2886 & 0.9669 & 0.2887 \\
\hline 85 & 0.9568 & 0.2720 & 0.9568 & 0.2720 & 0.9568 & 0.2720 \\
\hline 86 & 0.9460 & 0.2551 & 0.9460 & 0.2551 & 0.9460 & 0.2551 \\
\hline 87 & 0.9706 & 0.2902 & 0.9706 & 0.2902 & 0.9706 & 0.2902 \\
\hline 88 & 0.9590 & 0.2752 & 0.9590 & 0.2752 & 0.9590 & 0.2752 \\
\hline 89 & 0.9930 & 0.4075 & 0.9930 & 0.4075 & 0.9930 & 0.4075 \\
\hline 90 & 1.0161 & 0.3993 & 1.0161 & 0.3999 & 1.0161 & 0.4005 \\
\hline 91 & 1.0120 & 0.4288 & 1.0121 & 0.4304 & 1.0123 & 0.4321 \\
\hline 92 & 1.0196 & 0.4369 & 1.0196 & 0.4372 & 1.0197 & 0.4374 \\
\hline 93 & 1.0200 & 0.4787 & 1.0223 & 0.4826 & 1.0246 & 0.4864 \\
\hline 94 & 0.9856 & 0.3853 & 0.9869 & 0.3881 & 0.9882 & 0.3909 \\
\hline 95 & 0.9665 & 0.3970 & 0.9673 & 0.3997 & 0.9682 & 0.4025 \\
\hline 96 & 1.0035 & 0.4595 & 1.0035 & 0.4595 & 1.0035 & 0.4595 \\
\hline 97 & 1.0093 & 0.4645 & 1.0093 & 0.4645 & 1.0093 & 0.4645 \\
\hline 98 & 1.0605 & 0.4851 & 1.0609 & 0.4882 & 1.0613 & 0.4914 \\
\hline 99 & 0.9888 & 0.4734 & 0.9889 & 0.4736 & 0.9889 & 0.4737 \\
\hline 100 & 0.9846 & 0.4943 & 0.9846 & 0.4943 & 0.9846 & 0.4943 \\
\hline 101 & 0.9798 & 0.5385 & 0.9798 & 0.5385 & 0.9798 & 0.5385 \\
\hline 102 & 0.9867 & 0.5416 & 0.9867 & 0.5416 & 0.9867 & 0.5416 \\
\hline
\end{tabular}


Table 3.8 Estimated state variables and uncertainty bounds for the IEEE 118-bus network (continue)

\begin{tabular}{|l|l|l|l|l|l|l|}
\hline 103 & 0.9875 & 0.6201 & 0.9875 & 0.6201 & 0.9875 & 0.6201 \\
\hline 104 & 0.9875 & 0.5355 & 0.9875 & 0.5355 & 0.9875 & 0.5355 \\
\hline 105 & 0.9909 & 0.4980 & 0.9909 & 0.4980 & 0.9909 & 0.4980 \\
\hline 106 & 0.9813 & 0.4810 & 0.9813 & 0.4810 & 0.9813 & 0.4810 \\
\hline 107 & 0.9929 & 0.4781 & 0.9929 & 0.4781 & 0.9929 & 0.4782 \\
\hline 108 & 1.0115 & 0.4845 & 1.0115 & 0.4846 & 1.0116 & 0.4846 \\
\hline 109 & 1.0237 & 0.4762 & 1.0237 & 0.4762 & 1.0237 & 0.4762 \\
\hline 110 & 0.9928 & 0.5148 & 0.9928 & 0.5148 & 0.9928 & 0.5148 \\
\hline 111 & 0.9916 & 0.5618 & 0.9916 & 0.5618 & 0.9916 & 0.5618 \\
\hline 112 & 0.9612 & 0.3530 & 0.9612 & 0.3530 & 0.9612 & 0.3530 \\
\hline 113 & 0.9663 & 0.3365 & 0.9663 & 0.3365 & 0.9663 & 0.3365 \\
\hline 114 & 0.9671 & 0.3286 & 0.9671 & 0.3286 & 0.9671 & 0.3286 \\
\hline 115 & 0.9584 & 0.2549 & 0.9606 & 0.2611 & 0.9629 & 0.2673 \\
\hline 116 & 0.9589 & 0.2548 & 0.9605 & 0.2609 & 0.9621 & 0.2671 \\
\hline 117 & 0.9724 & 0.1803 & 0.9739 & 0.1971 & 0.9755 & 0.2139 \\
\hline 118 & 0.9490 & 0.3800 & 0.9496 & 0.3821 & 0.9503 & 0.3843 \\
\hline
\end{tabular}

\subsection{Summary}

Two formulations of uncertainty analysis in power system state estimation are presented in this study. The uncertainty is modeled via deterministic upper and lower bounds on measurement errors, which take into account known meter accuracies. Linear and nonlinear and formulations are provided to estimate the upper and lower bounds on the states. Both methods provided almost identical estimates, when applied to the six-bus test system. It is concluded from execution time analysis that (WLS-LP) is faster than (SQP) and more appropriate for uncertainty interval estimation in larger power networks. Consequently, analysis of uncertainty in power system state estimation with (WLS-LP) is applied on six-bus, IEEE 30-bus and the IEEE 118-bus test systems, for which a conventional WLS estimator is used to obtain point estimates of the states, and then a series of LP solutions is used to compute the tightest possible bounds on the states and 
other quantities of interest. The method offers useful additional information to the power system operator. By examining bounds on the estimates one can infer the quality of the metering configuration and determine the proximity of estimated quantities to voltage and flow limits with greater confidence. An interesting "cross-over" phenomena can occur in cases where network parameter errors exist, and this may be useful for model validation purposes.

It is important to stress that formulations proposed in this chapter assume that the transducer tolerances $\underline{\tau}$ must be known and fixed. In realty the instrument inaccuracies will become unknown as the instruments age under the action of various unknown processes and as the instruments are not recalibrated. It must be noted that measurement recalibration is rarely carried out in a systematic manner by utilities $[55,56]$. This is mainly due to the fact that large numbers of measurements exist in a power network and the time and expertise required to check each individual transducer would be expensive. 


\section{CHAPTER FOUR: ANALYSIS OF POWER SYSTEM STATE ESTIMATION WITH UNCERTAIN MEASUREMENTS AND PARAMETERS}

\subsection{Introduction}

The uncertainty analysis, in power system state estimation, can also be extended to other physical quantities such as the network parameters. In this chapter a parametric method for uncertainty analysis is proposed. The uncertainty is assumed to be present in both measurements and network parameters. Uncertainties in both measurements and parameters are known and bounded. The problem is formulated as a constrained nonlinear optimization problem. To find the tightest possible upper and lower bounds of any state variable, the problem is solved by Sequential Quadratic Programming (SQP) techniques. When applied to the six-bus, IEEE 14-bus and IEEE 30-bus networks, the proposed method shows reliability and accuracy in estimating the uncertainty bounds in power system state estimation. 


\subsection{Background}

In chapter 3, the uncertainty analysis of power system state estimation was strictly confined to the measurements received from the network. In fact, the uncertainties in the state estimates are not only due to the inaccuracies of the measurements. They are also due to the inaccuracies of the network mathematical model being used, which is expressed by $\underline{h}(\underline{x})$ in equation 3-1 (a vector of non-linear functions that relate the states to the measurements). These uncertainties originate from the approximations of the $\pi$ equivalent model of the network topology. Approximations of transmission lines and transformers, values of the resistances, reactances and shunt capacitances and the timeskew between the metered values are likely sources of inaccuracies of final network model (i.e. Y-admittance matrix).

Power line length and height above the ground also have a direct effect on the parameters (i.e. resistance, inductance and capacitance) [17]. According to [57, 58], factors like line loading, ambient temperature, wind speed and solar irradiation (direct sunshine) are known to affect the conductor heating (or, cooling) and, as a result, would cause variations in power line sag. Therefore, the transmission line parameters are susceptible to this variation. Which in turn, leads to some degree of uncertainty in their values. Consequently, this uncertainty in parameters should be considered in power system computation such as load-flow analysis and state estimation. 
Golub et al. have argued in [59], that optimization methods such as, regularized least squares (which is used to combat much of the ill-conditioning that arises in pure LS problems $[60,61]$ ), ridged regression and total least squares (which provides a way to deal with uncertainties in the data [62]) allow, in their various ways, the inclusion of a priori knowledge (probability distribution, for example) of the uncertain parameters in the problem at hand. Recently new algorithms have been proposed to solve the problem $A x \approx B$ in a total least square sense with uncertainty introduced in both the $A$ matrix and the $B$ vector. For instance, Chandrasekaran et al. in [63], has considered the case in which only selected columns of the coefficient matrix are subject to perturbations. The method guarantees that the effect of the uncertainties will never lead to an overestimation. On the other hand, the Structured Total Least Norm (STLN) described in [64], preserves the structure of the problem and minimizes the measure of the error in a discrete norm.

There has been little work on the effects of measurements and parametric uncertainty in engineering applications. In fact, concepts of uncertain estimation have been adopted mainly in the context of water distribution networks. Bargeila in [65], has adopted the ellipsoid method to provide confidence limit on state estimate the nonlinear water system. Nagar et al. [66] apply concepts from robust control theory and allowed for uncertainty in both the parameters and the measurements. The uncertainty is isolated with the use of a Linear Fractional Transformation (LFT), which enables the preservation of the structure of the uncertainty and allows for a separate manipulation of the nominal and uncertain part. The physical meaning of the LFT is described in figure 4.1 , where $M$ is the 
nominal matrix of the system at hand and $\Delta$ is a diagonal matrix where the uncertainties are isolated. In robust control this terminology called "pulling out the uncertainty" [67]. The LFT problem is an NP-hard problem. El Ghaoui and Calafiore in [68], have demonstrated that an LFT problem may be formulated as a convex semi-definite programming problem (SDP). A Linear Matrix Inequalities (LMI) [69] approach is then used to solve the (SDP) problem to obtain the upper and lower confidence bounds [70].

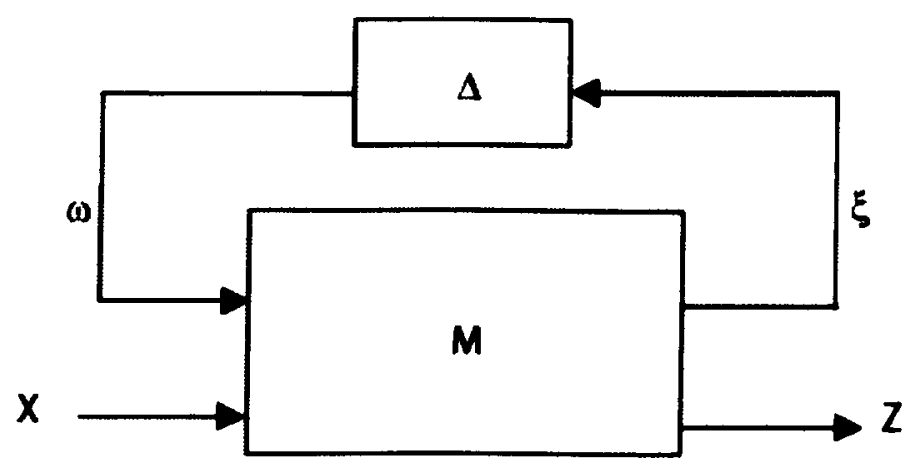

Figure 4.1 LFT representation where all the uncertainties isolated in $\Delta$.

This chapter proposes an alternative method for estimating the uncertainty interval around the system state variables. The method combines measurement and parameter uncertainties in a unified framework. A non-linear formulation is utilized to estimate the tightest possible upper and lower bounds on the states and the parameters. The estimation problem is then solved directly by means of Sequential Quadratic Programming (SQP). 


\subsection{Proposed Formulation}

Uncertainty intervals can be determined by the solution of a series of appropriately formulated optimization problems. Each measurement, with its associated uncertainty, can be represented by upper and lower limits. These constraint limits define the tolerances on the measurements (i.e. the range of values within which the true value of the measured quantity must lie). Minimizing a particular state variable of interest, subject to all the measurement inequality constraints, provides the lower bound on that state variable. Similarly, maximizing that state variable, again subject to all the measurement inequalities, provides the upper bound for that state. In mathematical form:

$$
\begin{gathered}
\min _{\underline{x}} x, \\
\text { subject to } \quad \underline{Z}^{\prime} \leq h(\underline{x}) \leq \underline{Z}^{\mu}
\end{gathered}
$$

where $\underline{Z}^{\prime}$ is the lower bound of the measurement vector and $\underline{Z}^{\mu}$ is upper bound, as described in equations 3-7 and 3-8 of chapter 3 .

The introduction of parametric uncertainty would clearly increase the dimensionality of the problem. The number of unknowns increases because the network parameters are no longer considered constants in the constraint evaluation phase (i.e. elements of the admittance matrix in power flow and injection equations must now be regarded as variables). The solver has to find the optimum values of not only the states, but also the network parameters that satisfy the available constraints. Hence, to account for 
parametric uncertainty in addition to measurement uncertainty the non-linear mathematical formulation in equation 4-1 would be extended to the following form:

$$
\min _{x, e}\left[x_{i} \text { or } \rho_{i}\right]
$$

$$
\begin{gathered}
\text { subject to } \quad \underline{Z}^{\prime} \leq H(\underline{x}, \underline{\rho}) \leq \underline{Z}^{u} \\
\underline{\rho}^{\prime} \leq \underline{\rho} \leq \underline{\rho}^{u}
\end{gathered}
$$

The new objective function is to minimize a particular element of interest contained in the vector $\underline{x}$ or in $\rho$, subject to all the measurement inequality constraints, to obtain a lower bound. Similarly, maximizing that element provides an upper bound. Vector $\underline{x}$ contains all the states (excluding the slack bus angle), which are arranged $\left[\theta_{2}, \ldots, \theta_{\mathrm{N}}, \mathrm{V}_{1}, \ldots, \mathrm{V}_{\mathrm{N}}\right]^{\mathrm{T}}$, where $N$ is the total number of nodes. Vector $\underline{\rho}$ includes all network transmission lines resistances $R$, reactances $X$ and the total line charging values $B$ (susceptance). Also, $\underline{Z}^{\prime}$ is the lower bound of the measurement vector and $\underline{Z}^{u}$ is upper bound. Furthermore, a set of double-sided inequality constraints has been added to the formulation, subjecting the varying (uncertain) transmission line parameters to a defined range. $\underline{\rho}^{l}$ And $\underline{\rho}^{u}$ are vectors of the lower and upper bounds on transmission line parameters respectively.

Clearly, the matrix $H$ could include the active and reactive power injections at any bus:

$$
\begin{aligned}
& P_{i}=V_{i} \sum_{j=1}^{N} V_{j}\left[G(\underline{\rho})_{i j} \cos \left(\theta_{i j}\right)+B(\underline{\rho})_{i j} \sin \left(\theta_{i j}\right)\right] \\
& Q_{1}=V_{i} \sum_{j=1}^{N} V_{j}\left[G(\underline{\rho})_{i j} \sin \left(\theta_{i j}\right)-B(\underline{\rho})_{i j} \cos \left(\theta_{i j}\right)\right]
\end{aligned}
$$


where $N$ is the total number of buses, $V_{i}$ is the magnitude of the bus $i$ voltage and $\theta_{i j}$ is the voltage phase angle between bus $i$ and bus $j$.

Also, any measured active and reactive line flows (sending end) are included as:

$$
\begin{gathered}
P_{i j}=V_{i}^{2} G(\underline{\rho})_{i j}-V_{i} V_{j} G(\underline{\rho})_{i j} \cos \left(\theta_{i j}\right)+V, V_{j} B(\underline{\rho})_{i j} \sin \left(\theta_{i j}\right) \\
i=1,2,3 \ldots \ldots ., N \quad \text { and } \quad j=1,2,3 \ldots \ldots, N \\
Q_{i j}=-V_{i}^{2}\left[B(\underline{\rho})_{i j}+B(\underline{\rho})_{i j}^{s h}\right]+V, V_{j} B(\underline{\rho})_{i j} \cos \left(\theta_{i j}\right)+V, V, G(\underline{\rho})_{i j} \sin \left(\theta_{i j}\right) \\
i=1,2,3 \ldots \ldots ., N \quad \text { and } \quad j=1,2,3 \ldots \ldots ., N
\end{gathered}
$$

It is important to note that the elements of admittance matrix, $G_{i j}$ and $B_{i j}$, are no longer constants when calculating power flows and injections. Equations 4-3 to 4-6 are a function of the state variables and the varying network parameters contained in $\underline{\rho}$. As a result, when the solver iterates on the unknowns of the optimization problem, an update of the admittance matrix has to be recomputed for proper evaluation of equations. 4-3 to 4-6. With modern high speed computers and utilizing sparsity techniques, this issue poses no significant computational burden. 


\subsection{Overview of non-linear optimization:}

The main objective in a constrained optimization problem is to transform the problem into subproblem that can be handled without very much difficulty. That subproblem can then be solved and used as the basis of an iterative process [71]. Occasionally, translation of the constrained problem to a basic unconstrained problem is achieved using penalty functions for constraints that are near or outside the constraint boundary. Then the constrained problem is solved using a sequence of parameterized unconstrained optimizations, which converge to the solution of the constrained problem.

These ways of solving constrained optimization problems are now considered relatively ineffective, in some situations, and inaccurate. As an alternative, optimization methods have focused on the solution of the Kursh-Kuhn-Tucker (KKT) equations. The KKT equations are necessary conditions for optimality for a constrained optimization problem. If the problem (both the objective function and the constraints) at hand is convex, then the $\mathrm{KT}$ equations are necessary and sufficient for finding a global solution point. The Kuhn-Tucker equations can be formulated as:

$$
\begin{aligned}
& \nabla f\left(x^{*}\right)+\sum_{i=1}^{m} \lambda_{i} * \nabla g_{i}\left(x^{*}\right)=0 \\
& \lambda_{i}{ }^{*} \nabla g_{i}\left(x^{*}\right)=0 \quad i=1, \ldots . ., m_{e} \\
& \lambda_{i} \geq 0 \quad i=m_{e}+1, \ldots . ., m
\end{aligned}
$$

where

$f(x)$ is the objective function.

$g(x)$ is the constraints of the objective function. 
$\lambda \quad$ is the Lagrange multiplier.

$\nabla \quad$ is the gradient.

$m_{e} \quad$ is the number of equality constraint.

$m \quad$ is the number of inequality constraint.

The first equation is necessary for cancelling gradients between the objective function and the active constraints at the solution point $x^{\circ}$. In order to cancel out the gradients, the Lagrange multipliers, i.e. $\lambda_{i} \quad, i=1, \ldots . ., m$ are necessary to balance the deviations, or incremental changes, in the objective function magnitude and constraint gradients. Only active constraints must be included in this operation. Lagrange multipliers of non-active constraints must be set equal to zero.

The non-linear programming method used is based on Powell's algorithm [72, 73], which solves a sequence of positive definite quadratic programming subproblems. The nonlinear programming method can be geometrically interpreted as finding a solution point within all the measurement constraints hyperplanes. The estimated values lie at the intersection of $p$ hyperplanes in $p$ dimensional space, where the estimator will select a set of $p$ hyperplanes from the $n_{m}$ available values to minimize the objective function. Therefore, the bounding $p$ measurements are selected to define the solution point $[12$, 74]. 


\subsubsection{Sequential Quadratic Programming (SQP)}

The solution of the $\mathrm{KT}$ equations forms the basis of many nonlinear programming algorithms. These algorithms attempt to compute the Lagrange multipliers directly. Constrained quasi-Newton methods guarantee superlinear convergence by accumulating second order information regarding the KT equations using a quasi-Newton updating procedure [75]. These methods are commonly referred to as Sequential Quadratic Programming (SQP) methods, since a $\mathrm{QP}$ subproblem is solved at each major iteration (also known as Iterative Quadratic Programming, Recursive Quadratic Programming, and Constrained Variable Metric methods). The QP optimization problem can be described as follows:

Where

$$
\begin{aligned}
& \min \left(\frac{1}{2} d_{k}^{T} H_{k} d_{k}+\nabla f\left(x_{k}\right)^{T} d_{k}\right) \\
& \text { suject to } \quad\left[\nabla g\left(x_{k}\right)\right]^{T} d_{k}+g_{i}\left(x_{k}\right)=0 \quad i=1, \ldots . ., m_{e} \\
& {\left[\nabla g\left(x_{k}\right)\right]^{T} d_{k}+g_{i}\left(x_{k}\right) \leq 0 \quad i=m_{e}+1, \ldots . ., m}
\end{aligned}
$$

$H_{k} \quad$ is the Hessian matrix of the Lagrange function $L(x, \lambda)=f(x)+\sum_{i=1}^{m} \lambda_{1}^{*} g_{1}(x)$.

$d_{k} \quad$ is a basis of the search direction of the $\mathrm{k}^{\text {th }}$ iteration.

SQP can be decomposed into three main stages:

- Updating of the Hessian matrix of the Lagrangian function.

- Line search and merit function calculation. 
- Quadratic programming problem solution.

A convergence test is made at each iteration, after the solution of the quadratic programming problem until the control variables, gradient of functions and objective function reaches a specified tolerance value $[72,76]$.

\subsection{Results and analysis}

This section presents some typical results obtained by applying the proposed algorithms on three sample power systems. The first system has six buses, is originally obtained from reference [41] and shown in figure 2.2. The second system is the IEEE 14-bus test network [77], shown in figure 4.2. The third system is the IEEE 30-bus test network [77] which is shown in figure 4.3. The associated network data of all test systems are provided in the appendix.

The computation of all state variables and all parameters will be shown to illustrate the concepts for the six-bus and IEEE 14-bus systems. However, for improved computational efficiency, only the variables of present interest to the power system operator would need to be computed. The non-linear problems have been solved by the function fmincon incorporated in the MATLAB ${ }^{\mathrm{TM}} 6.1$ optimization toolbox [49]

As an application of non linear programming in power system state estimation, Abbasy and Shahidehpour have employed (SQP) to estimate the states of five-bus network in 
[74]. Outcome of (SQP) was identical to those obtained from (LP) and (WLS).

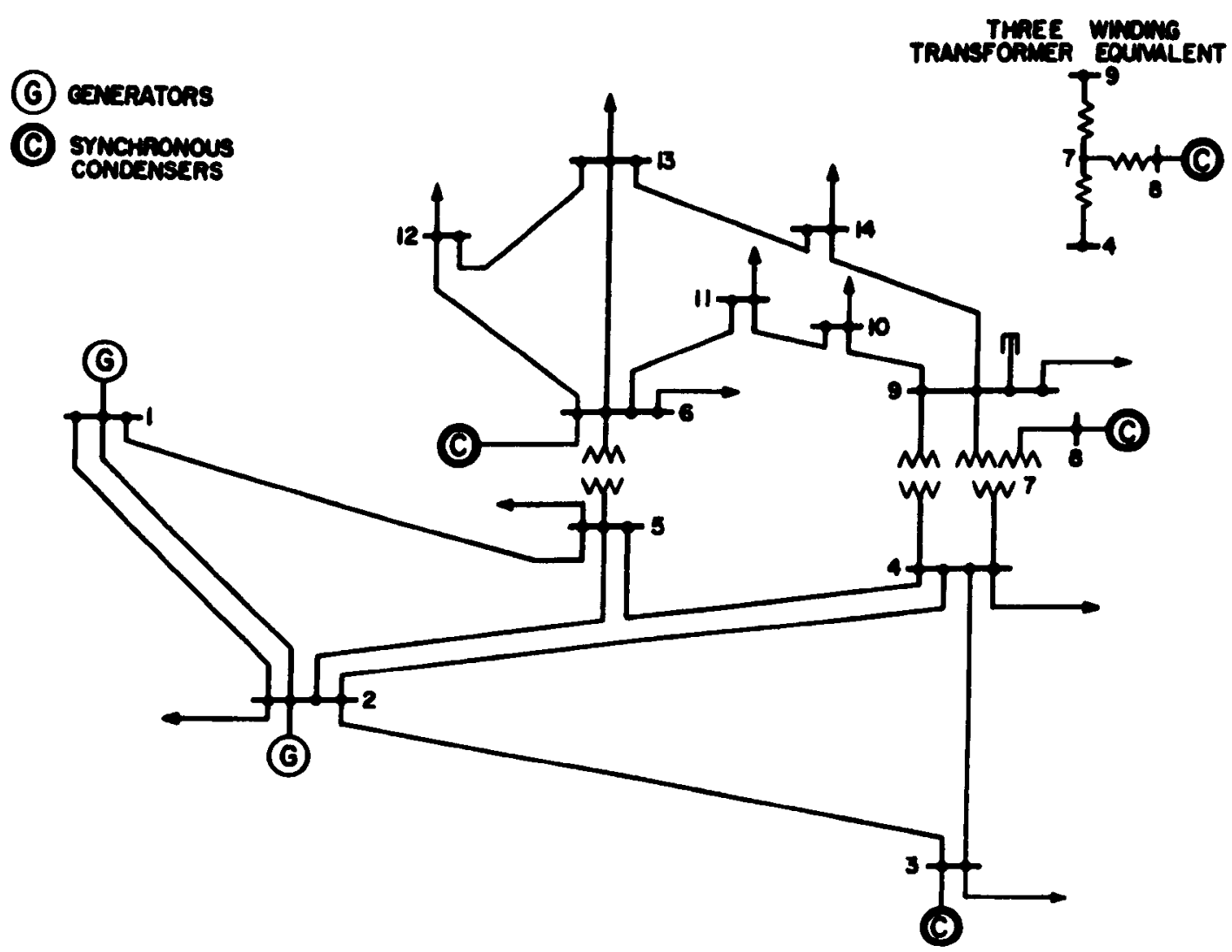

Figure 4.2 IEEE 14-bus test system

\subsubsection{Estimation of states confidence bounds}

To demonstrate the effect of parametric uncertainty on the estimation process, a test with fixed (certain) measurements and uncertain parameters is carried out on the six-bus test system. All parameters in $\underline{\rho}$ are permitted to vary within a range of $\pm 2 \%$ of their nominal values. The outcome is shown in table 4.1. Estimation of the states with conventional WLS (to define a central point estimate) are included for comparative 
purposes with the SQP estimates. Results in table 4.1 are illustrated in figures 4-4 and 45.

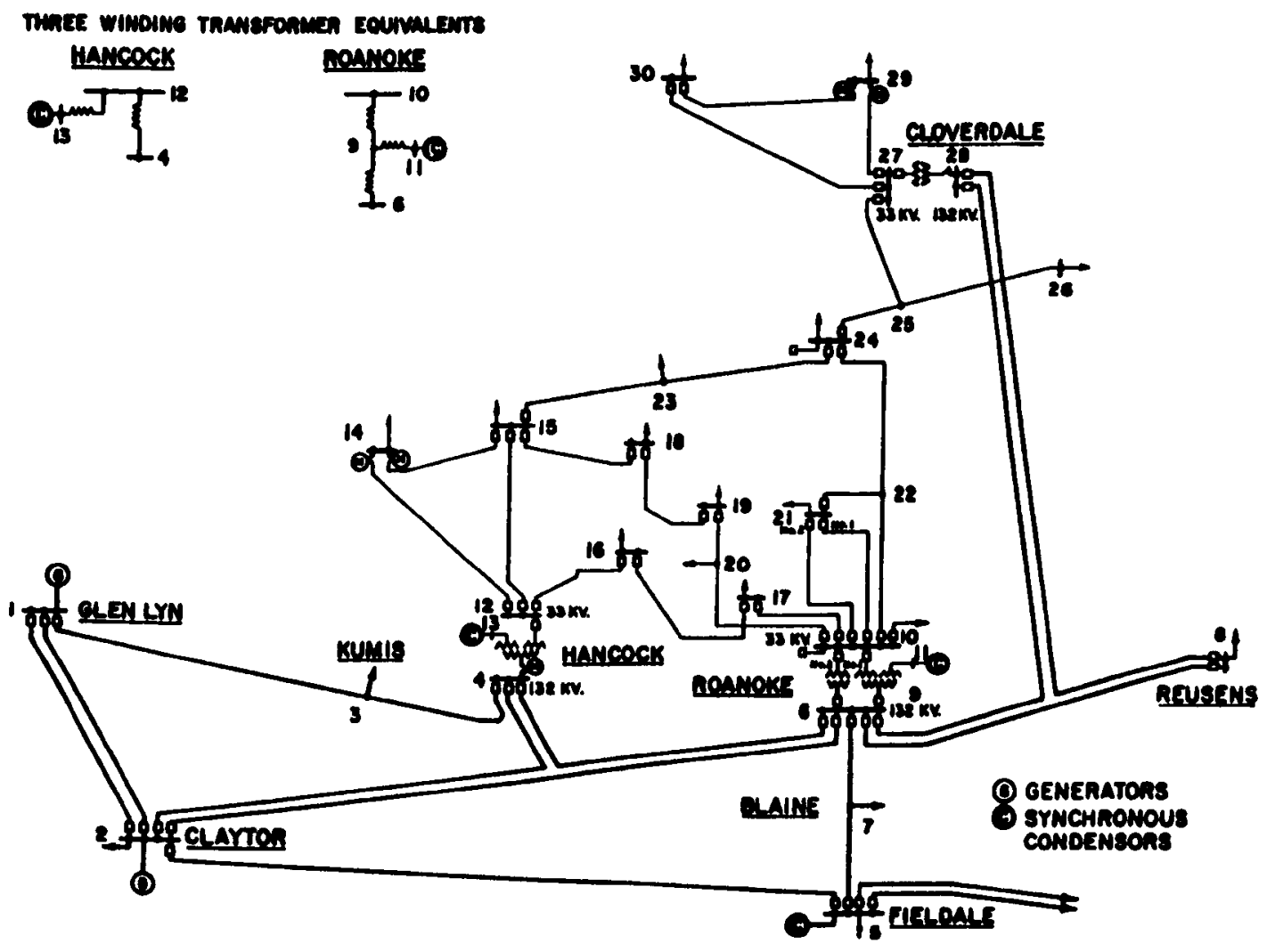

Figure 4.3 IEEE 30-bus test system 
Table 4.1 Estimated state variables with uncertain parameters for the six-bus system

\begin{tabular}{|c|c|c|c|c|c|c|}
\hline \multirow{2}{*}{ Bus \# } & \multicolumn{2}{|c|}{$\mathbf{S Q P}_{\text {(lower bound) }}^{-}$} & \multicolumn{2}{c|}{$\mathbf{W L S}_{\text {(centre) }}$} & \multicolumn{2}{c|}{$\mathbf{S Q P}_{\text {(upper bound) }}$} \\
\cline { 2 - 7 } & $|\mathbf{V}|(\mathrm{pu})$ & $\boldsymbol{\delta}(\mathrm{r}$ ad) & $|\mathbf{V}|(\mathrm{pu})$ & $\boldsymbol{\delta}(\mathrm{r}$ ad) & $|\mathbf{V}|(\mathrm{pu})$ & $\boldsymbol{\delta}(\mathrm{r}$ ad $)$ \\
\hline $\mathbf{1}$ & 1.0475 & 0 & 1.0500 & 0 & 1.0525 & 0 \\
\hline $\mathbf{2}$ & 1.0481 & -0.0675 & 1.0500 & -0.0650 & 1.0519 & -0.0625 \\
\hline $\mathbf{3}$ & 1.0674 & -0.0791 & 1.0700 & -0.0756 & 1.0727 & -0.0722 \\
\hline $\mathbf{4}$ & 0.9841 & -0.0754 & 0.9864 & -0.0729 & 0.9887 & -0.0705 \\
\hline $\mathbf{5}$ & 0.9777 & -0.0940 & 0.9797 & -0.0912 & 0.9817 & -0.0884 \\
\hline $\mathbf{6}$ & 0.9989 & -0.1076 & 1.0014 & -0.1042 & 1.0040 & -0.1008 \\
\hline
\end{tabular}

From figure 4-2 and 4-3, we notice that solution provided by WLS is strictly bounded by the solution of SQP.

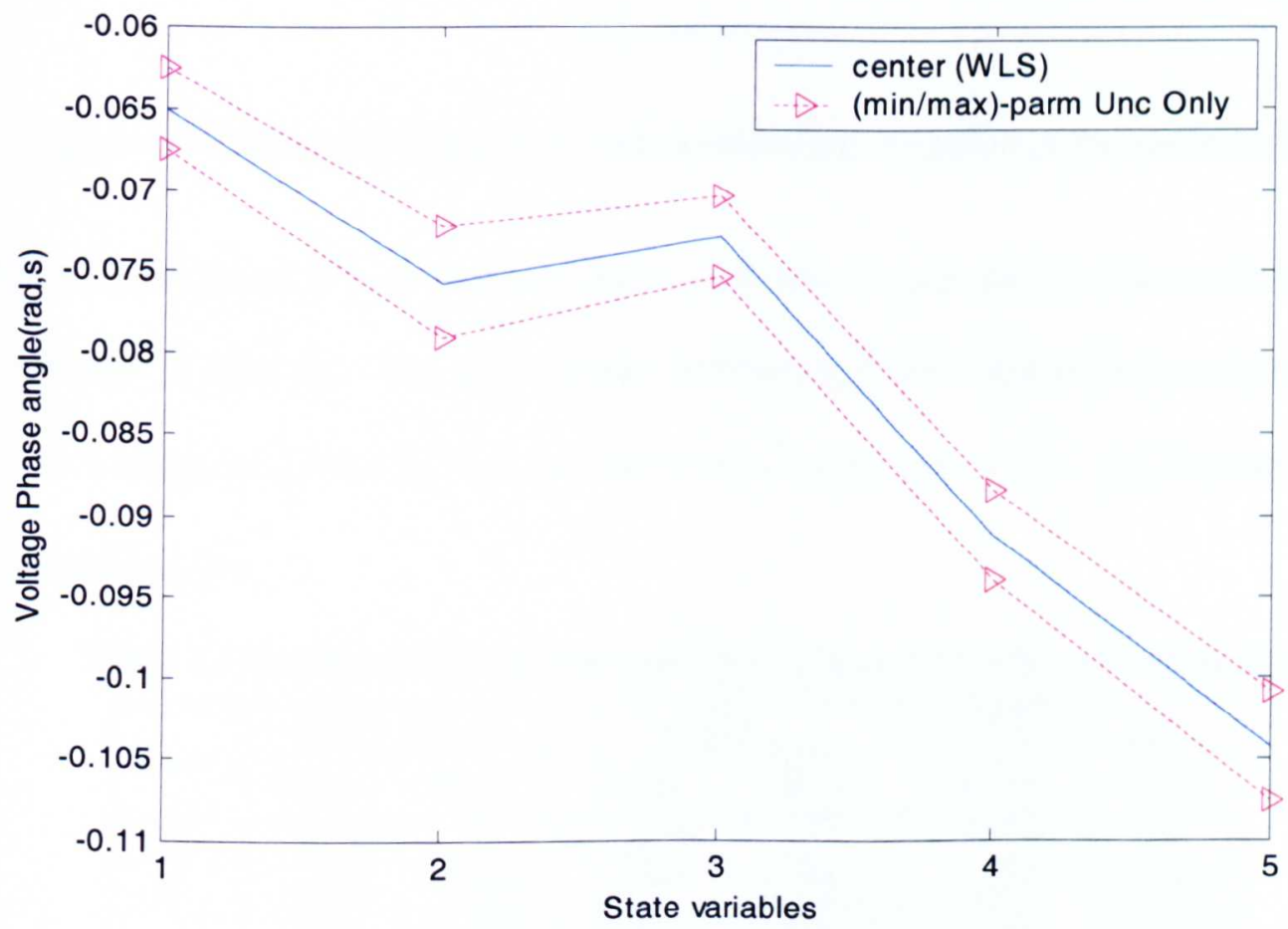

Figure 4.4 Estimated phase angles and uncertainty bounds for the six-bus system 


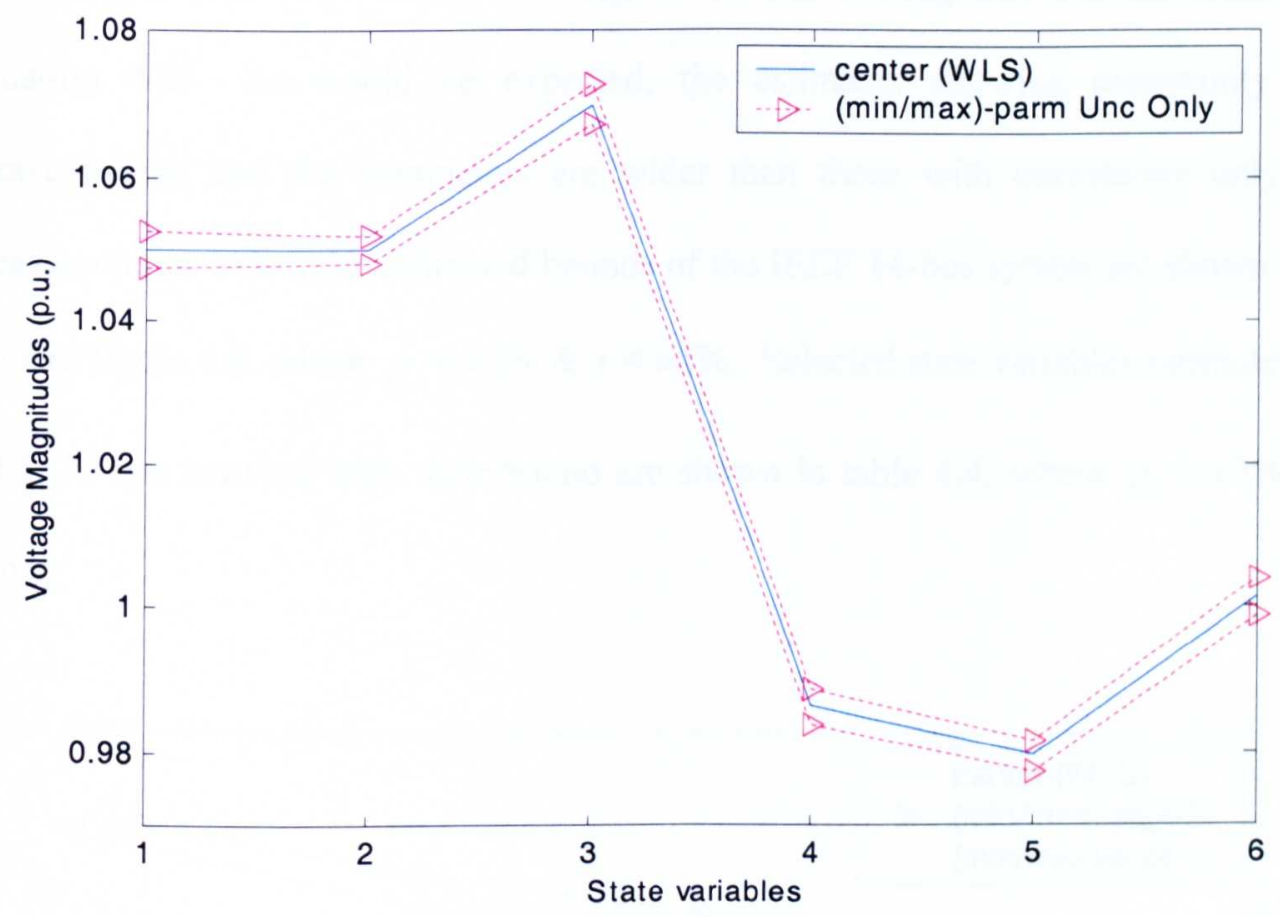

Figure 4.5 Estimated magnitudes and uncertainty bounds for the six-bus system

When uncertainties in both measurements and parameters are present, the results obtained are shown in table 4-2. The deterministic uncertainty in the measurements is assumed to cover a range of $[-3 \%, 3 \%]$ of nominal values, while parametric uncertainties $\underline{\rho}$ are bounded by $\pm 2 \%$.

Table 4.2 Estimated states with uncertain measurements and parameters

\begin{tabular}{|c|c|c|c|c|c|c|}
\hline \multirow{2}{*}{ Bus \# } & \multicolumn{2}{|c|}{$\mathbf{S Q P}_{\text {(lower bound) }}^{-}$} & \multicolumn{2}{c|}{$\mathbf{W L S}_{\text {(centre) }}$} & \multicolumn{2}{c|}{$\mathbf{S Q P}_{\text {(upper bound) }}$} \\
\cline { 2 - 7 } & $|\mathbf{V}|(\mathrm{pu})$ & $\boldsymbol{\delta}(\mathrm{r}$ ad $)$ & $|\mathbf{V}|(\mathrm{pu})$ & $\boldsymbol{\delta}(\mathrm{r} a d)$ & $|\mathbf{V}|(\mathrm{pu})$ & $\boldsymbol{\delta}(\mathrm{r}$ ad $)$ \\
\hline \hline $\mathbf{1}$ & 1.0178 & 0 & 1.0500 & 0 & 1.0818 & 0 \\
\hline $\mathbf{2}$ & 1.0235 & -0.0818 & 1.0500 & -0.0650 & 1.0762 & -0.0495 \\
\hline $\mathbf{3}$ & 1.0436 & -0.0981 & 1.0700 & -0.0756 & 1.0960 & -0.0549 \\
\hline $\mathbf{4}$ & 0.9584 & -0.0887 & 0.9864 & -0.0729 & 1.0141 & -0.0586 \\
\hline $\mathbf{5}$ & 0.9533 & -0.1105 & 0.9797 & -0.0912 & 1.0058 & -0.0735 \\
\hline $\mathbf{6}$ & 0.9757 & -0.1275 & 1.0014 & -0.1042 & 1.0268 & -0.0827 \\
\hline
\end{tabular}


The result of table 4.2 is illustrated in figures 4.6 and 4.7 , together with the solution from equation 4-2. As would be expected, the estimates allowing uncertainty in the measurements and the parameters are wider than those with uncertainty only in the measurements. Also, the estimated bounds of the IEEE 14-bus system are shown in table 4.3 and figure 4.8 , where $\underline{\rho}= \pm 2 \% \& \tau= \pm 6 \%$. Selected state variables estimates of the IEEE 30-bus network with their bound are shown in table 4.4, where $\underline{\rho}= \pm 3 \% \& \tau=$ $\pm 10 \%$.

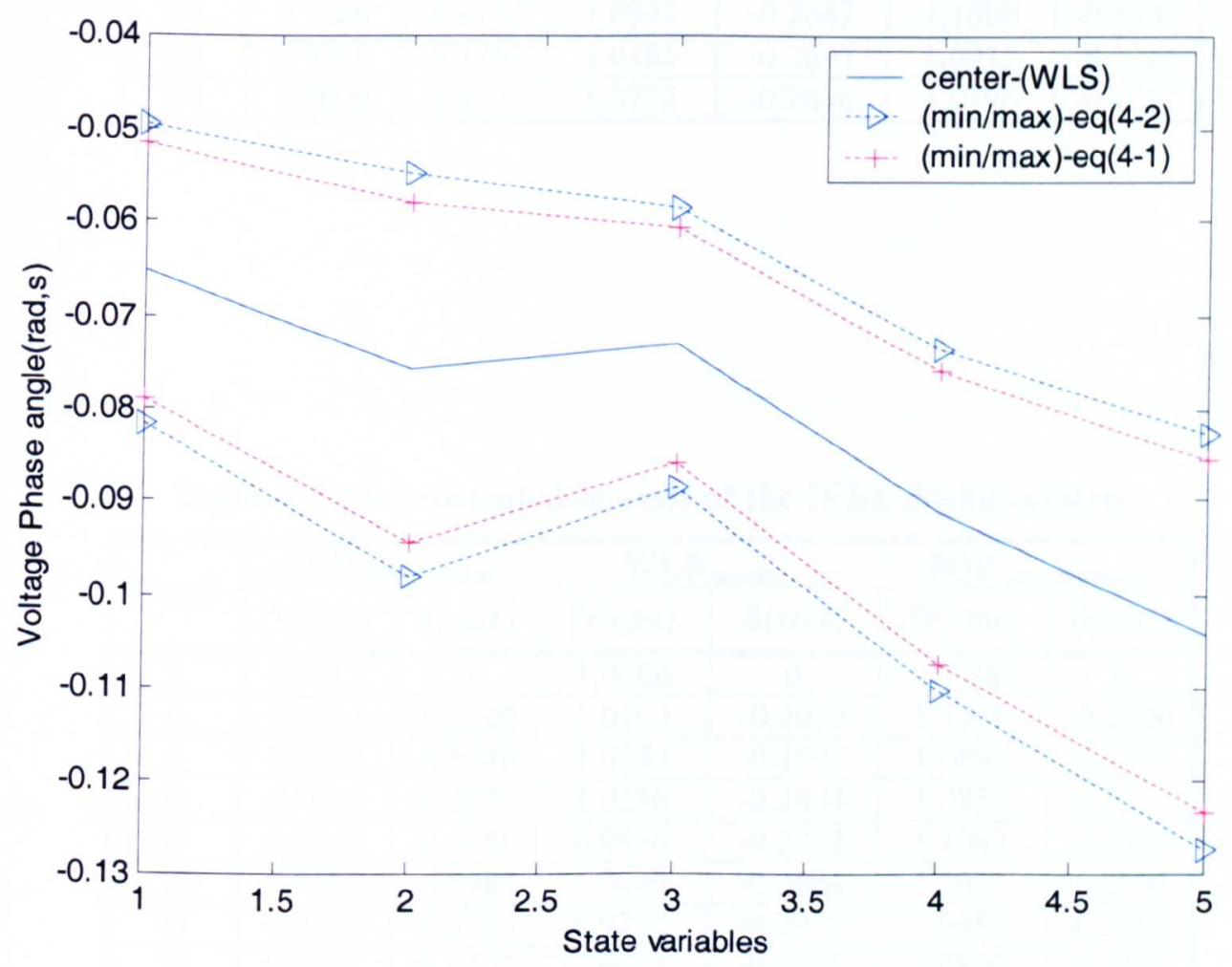

Figure 4.6 Estimated phase angles and uncertainty bounds for the six-bus system 
Table 4.3 The estimated bounds of the IEEE 14-bus system

\begin{tabular}{|c|c|c|c|c|c|c|}
\hline \multirow{2}{*}{ Bus \# } & \multicolumn{2}{|c|}{$\mathbf{S Q P}_{\text {(lower bound) }}^{-}$} & \multicolumn{2}{c|}{ WLS $_{\text {(centre) }}$} & \multicolumn{2}{c|}{$\mathbf{S Q P}_{\text {(upper boumd) }}^{+}$} \\
\cline { 2 - 7 } & $|\mathbf{V}|(\mathrm{pu})$ & $\boldsymbol{\delta}(\mathrm{rad})$ & $|\mathbf{V}|(\mathrm{pu})$ & $\boldsymbol{\delta}(\mathrm{rad})$ & $|\mathbf{V}|(\mathrm{pu})$ & $\boldsymbol{\delta}(\mathrm{rad})$ \\
\hline $\mathbf{1}$ & 0.9314 & 0 & 1.0600 & 0 & 1.1000 & 0 \\
\hline $\mathbf{2}$ & 0.9239 & -0.1454 & 1.0450 & -0.0859 & 1.0959 & -0.0514 \\
\hline $\mathbf{3}$ & 0.9000 & -0.3405 & 1.0100 & -0.2181 & 1.0696 & -0.1510 \\
\hline $\mathbf{4}$ & 0.9269 & -0.3083 & 1.0427 & -0.1839 & 1.0933 & -0.1103 \\
\hline $\mathbf{5}$ & 0.9319 & -0.2725 & 1.0514 & -0.1601 & 1.1000 & -0.0933 \\
\hline $\mathbf{6}$ & 0.9298 & -0.4434 & 1.0700 & -0.2539 & 1.1000 & -0.1357 \\
\hline $\mathbf{7}$ & 0.9114 & -0.4141 & 1.0500 & -0.2353 & 1.0698 & -0.1239 \\
\hline $\mathbf{8}$ & 0.9464 & -0.4240 & 1.0900 & -0.2353 & 1.1000 & -0.1153 \\
\hline $\mathbf{9}$ & 0.9000 & -0.4550 & 1.0429 & -0.2629 & 1.0795 & -0.1422 \\
\hline $\mathbf{1 0}$ & 0.9000 & -0.4671 & 1.0401 & -0.2663 & 1.0839 & -0.1391 \\
\hline $\mathbf{1 1}$ & 0.9464 & -0.4659 & 1.0514 & -0.2622 & 1.0963 & -0.1334 \\
\hline $\mathbf{1 2}$ & 0.9000 & -0.4787 & 1.0542 & -0.2687 & 1.1000 & -0.1341 \\
\hline $\mathbf{1 3}$ & 0.9000 & -0.4761 & 1.0485 & -0.2697 & 1.0932 & -0.1386 \\
\hline $\mathbf{1 4}$ & 0.9000 & -0.4977 & 1.0272 & -0.2836 & 1.0796 & -0.1477 \\
\hline
\end{tabular}

Table 4.4 The estimated bounds of the IEEE 30-bus system

\begin{tabular}{|c|c|c|c|c|c|c|}
\hline \multirow{2}{*}{ Bus \# } & \multicolumn{2}{|c|}{$\mathbf{S Q P}_{\text {(lower bound) }}^{-}$} & \multicolumn{2}{c|}{ WLS $_{\text {(centre) }}$} & \multicolumn{2}{c|}{$\mathbf{S Q P}^{+}{ }_{\text {(upper bound) }}$} \\
\cline { 2 - 7 } & $|\mathbf{V}|(\mathrm{pu})$ & $\boldsymbol{\delta}(\mathrm{r} a d)$ & $|\mathbf{V}|(\mathrm{pu})$ & $\boldsymbol{\delta}(\mathrm{r} a d)$ & $|\mathrm{V}|(\mathrm{pu})$ & $\boldsymbol{\delta}(\mathrm{rad})$ \\
\hline $\mathbf{1}$ & 0.9413 & 0 & 1.0600 & 0 & 1.1300 & 0 \\
\hline $\mathbf{8}$ & 0.9564 & -0.3200 & 1.0100 & -0.2057 & 1.1330 & -0.2000 \\
\hline $\mathbf{9}$ & 0.9033 & -0.5540 & 1.0364 & -0.2507 & 1.0895 & -0.1454 \\
\hline $\mathbf{1 0}$ & 0.9047 & -0.3675 & 1.0256 & -0.2804 & 1.0855 & -0.1541 \\
\hline $\mathbf{2 7}$ & 0.9464 & -0.5658 & 0.9956 & -0.2752 & 1.1063 & -0.2334 \\
\hline $\mathbf{2 8}$ & 0.9300 & -0.5787 & 1.0099 & -0.2044 & 1.1033 & -0.2991 \\
\hline $\mathbf{2 9}$ & 0.9210 & -0.4760 & 0.9752 & -0.2979 & 1.0982 & -0.3006 \\
\hline $\mathbf{3 0}$ & 0.9001 & -0.4707 & $\mathbf{0 . 9 6 3 3}$ & -0.3142 & 1.0890 & -0.3505 \\
\hline
\end{tabular}




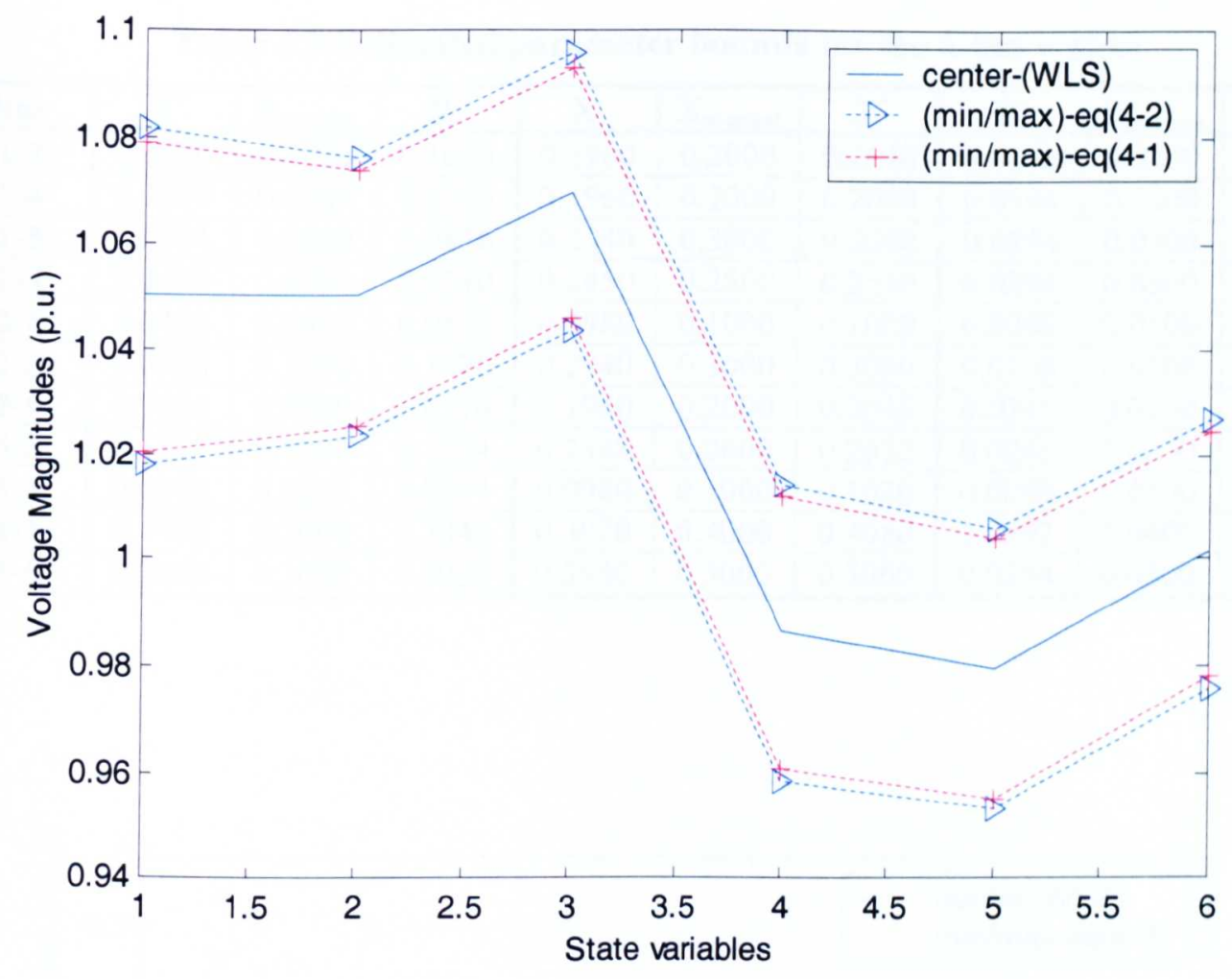

Figure 4.7 Estimated magnitudes and uncertainty bounds for the six-bus system

\subsubsection{Estimation of confidence bounds on parameters}

Equation (4-2) is also capable of estimating (minimize or maximize) any chosen parameter. Table 4.5 shows the nominal parameters of the each line (The six-bus network has 11 lines) with associated upper and lower bound. 
Table 4.5 Estimated parameter bounds for the 6-bus system

\begin{tabular}{|c||c|c|c|c|c|c|c|c|c|}
\hline line & $\mathbf{R}^{-}$ & $\mathbf{R}_{\text {nominal }}$ & $\mathbf{R}^{+}$ & $\mathbf{X}^{-}$ & $\mathbf{X}_{\text {nominal }}$ & $\mathbf{X}^{+}$ & $\mathbf{B}^{-}$ & $\mathbf{B}_{\text {nominal }}$ & $\mathbf{B}^{+}$ \\
\hline $\mathbf{1 - 2}$ & 0.0980 & 0.1000 & 0.1020 & 0.1960 & 0.2000 & 0.2040 & 0.0196 & 0.0200 & 0.0204 \\
\hline $\mathbf{1 - 4}$ & 0.0490 & 0.0500 & 0.0510 & 0.1960 & 0.2000 & 0.2040 & 0.0196 & 0.0200 & 0.0204 \\
\hline $\mathbf{1 - 5}$ & 0.0784 & 0.0800 & 0.0816 & 0.2940 & 0.3000 & 0.3060 & 0.0294 & 0.0300 & 0.0306 \\
\hline $\mathbf{2 - 3}$ & 0.0490 & 0.0500 & 0.0510 & 0.2450 & 0.2500 & 0.2550 & 0.0294 & 0.0300 & 0.0306 \\
\hline $\mathbf{2 - 4}$ & 0.0490 & 0.0500 & 0.0510 & 0.0980 & 0.1000 & 0.1020 & 0.0098 & 0.0100 & 0.0102 \\
\hline $\mathbf{2 - 5}$ & 0.0980 & 0.1000 & 0.1020 & 0.2940 & 0.3000 & 0.3060 & 0.0196 & 0.0200 & 0.0204 \\
\hline $\mathbf{2 - 6}$ & 0.0686 & 0.0700 & 0.0714 & 0.1960 & 0.2000 & 0.2040 & 0.0245 & 0.0250 & 0.0255 \\
\hline $\mathbf{3 - 5}$ & 0.1176 & 0.1200 & 0.1224 & 0.2548 & 0.2600 & 0.2652 & 0.0245 & 0.0250 & 0.0255 \\
\hline $\mathbf{3 - 6}$ & 0.0196 & 0.0200 & 0.0204 & 0.0980 & 0.1000 & 0.1020 & 0.0098 & 0.0100 & 0.0102 \\
\hline $\mathbf{4 - 5}$ & 0.1960 & 0.2000 & 0.2040 & 0.3920 & 0.4000 & 0.4080 & 0.0392 & 0.0400 & 0.0408 \\
\hline $\mathbf{5 - 6}$ & 0.0980 & 0.1000 & 0.1020 & 0.2940 & 0.3000 & 0.3060 & 0.0294 & 0.0300 & 0.0306 \\
\hline
\end{tabular}
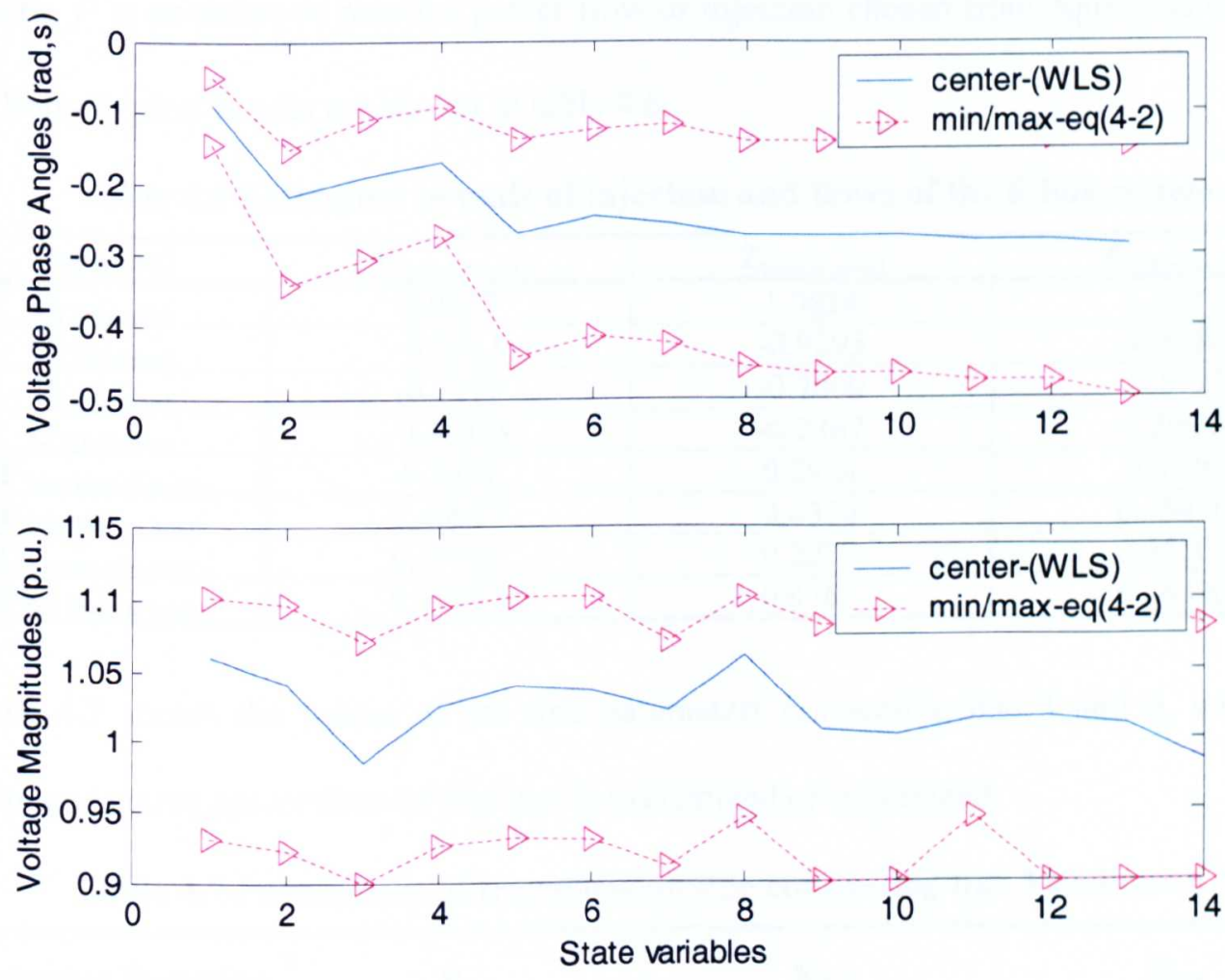

Figure 4.8 Estimated states and uncertainty bounds for the IEEE 14-bus system 


\subsubsection{Estimation of other quantities}

In addition to solving for the uncertainty ranges of the state variables (voltage magnitudes and phase angles) or the network parameters, it is also possible to compute the uncertainty range of other estimated quantities (such as power flows and injections), whether these quantities are measured or not. For example, with a change in the objective functions of (4-2), the lower bound of the $t^{\text {th }}$ measurement could be found by solving the following minimization problem:

$$
\min _{x, e} f_{i}(x, \rho)
$$

subject to

$$
\begin{gathered}
\underline{Z}^{\prime} \leq H(\underline{x}, \underline{\rho}) \leq \underline{Z}^{\mu} \\
\underline{\rho}^{\prime} \leq \underline{\rho} \leq \underline{\rho}^{\prime \prime}
\end{gathered}
$$

where $f$ is an active or reactive power flow or injection chosen from equations 4-3 to 4-

\begin{tabular}{|c|c|c|c|}
\hline Type (pu) & $\mathbf{Z}_{\text {(lower bound) }}$ & $\mathbf{Z}_{\text {(conter point) }}$ & $\mathbf{Z}_{\text {(upper bound) }}$ \\
\hline $\mathbf{P}_{\text {1iniection) }}$ & 0.9337 & 1.0824 & 1.2375 \\
\hline $\mathbf{Q}_{1 \text { (iniection) }}$ & -0.9543 & -0.9295 & -0.8986 \\
\hline $\mathbf{P}_{5 \text { (iniection) }}$ & -0.7277 & -0.7000 & -0.6722 \\
\hline $\mathbf{Q}_{3 \text { (iniection) }}$ & -0.26453 & -0.2367 & -0.20894 \\
\hline $\mathbf{P}_{\text {line (bual to bus2) }}$ & 0.2924 & 0.2924 & 0.3140 \\
\hline$P_{\text {line (busl to bust) }}$ & 0.40917 & 0.4370 & 0.46476 \\
\hline $\mathbf{Q}_{\text {line (bus } 4 \text { to busl) }}$ & 0.19948 & 0.2273 & 0.25507 \\
\hline $\mathbf{P}_{\text {line (bur } 3 \text { lo hur 6) }}$ & 0.40838 & 0.4362 & 0.46396 \\
\hline
\end{tabular}

6. Some typical results are shown in table 4.6.

Table 4.6 Estimated bounds of injection and nows of the 6-bus system

Table 4.7 shows the values of the line parameters connecting bus 3 and 6 , when the

\begin{tabular}{|c|c|c|c|}
\hline Objective Function & $\mathbf{R}_{3.6}$ & $X_{3-6}$ & $\mathbf{B}_{3-6}$ \\
\hline $\operatorname{Max}\left(P_{\text {line (bus3 to bur6) }}\right)$ & 0.0204 p.u & 0.0995 p.u & 0.0102 p.u \\
\hline Min ( $\mathbf{P}_{\text {line }}$ (bues to bus 6$)$ & 0.0196 p.u & 0.1020 p.u & 0.0101 p.u \\
\hline
\end{tabular}
estimated active power flow of that line is maximized or minimized.

Table 4.7 Parameters of transmission line connecting bus 3 and bus 6 . 


\subsection{Summary}

An analysis of uncertainty in power system state estimation is presented in this chapter. The uncertainty is modelled via deterministic upper and lower bounds on measurement errors, which take into account known meter accuracies. Parametric uncertainties are also known and bounded. A non-linear formulation is provided to estimate the upper and lower bounds on the states and the parameters. The non-linear problem is solved by Sequential Quadratic Programming (SQP) techniques. The method offers useful additional information to the power system operator. By examining bounds on the estimates one can infer the quality of the metering configuration and determine the proximity of estimated quantities to voltage and flow limits with greater confidence. 


\section{CHAPTER FIVE: ROBUST STATE ESTIMATION}

\subsection{Introduction}

In traditional power system state estimation, estimators based on statistical methods such as least squares and least absolute values, along with their variants, are well documented and have been widely applied. Researchers have begun to realize that measurements acquired from the field usually do not entirely satisfy the assumptions on which those estimators have been initially bulit. Circumstances such as faulty measurements pose a serious threat to the quality of the estimator outcome. Hence, robust estimators have been introduced as an alternative, to eliminate or down-weigh the effects of faulty measurements (or outliers).

Indeed, the uncertainty interval estimation methods presented in previous chapters assume that measurements obtained from the power network are filtered and error free (i.e., there exist no outliers or leverage points). Otherwise, an attempt to solve either the linear or the non-linear optimisation problems would result in either failure due to infeasibility or production of erroneous bounds.

In this chapter, robust methods developed in statistics literature are reviewed. The wellknown Least Median Square (LMS) robust estimator [78, 79] and its application in power systems [80-83] are revisited. A comparison between the LMS and LS is carried out to show effectiveness in the presence of outliers. It is also shown how collinearity in the 
measurements undermines the performance of LMS estimator. A new robust estimator based on the concept of uncertainty in the measurements is developed here. This estimator is known as Maximum Constraints Satisfaction (MCS). Robustness and performance of the proposed estimator is discussed via simulated problems of simple regression examples, D.C. three-bus system and the six-bus test system.

\subsection{Background}

In statistics and regression analysis, an outlier is an observation that is inconsistent with the remainder of the measurements. In other words, the corresponding error or residual is large compared with those of the majority of the other observations. Theoretically, one can identify whether the observations are outliers or not, only after finding the regression coefficients (or the fit) from which a residual analysis could be carried out for identification.

Generally, outliers occur quite frequently, as a consequence of high amplitude noise in measurements (e.g., due to induction during large transients), brief loss of measurement data and noise occurring due to unintended signal paths and measurements [84]. Furthermore, many AC applications result in frequent measurement errors and "periodic noise". Periodic errors are common in power electronic switched applications.

As far as state estimation is concerned, when the measurement noise is modelled as Gaussian with a given covariance, the least squares estimator gives the maximum- 
likihood minimum variance estimates. In practice however, due to the massive amount of metering that exists in a large power network, it is very highly likely that the noise statistics are not accurately determined. Therefore, if some of the measurements are extremely contaminated, estimation by the least squares estimator will give deceptive results. Consequently, for a proper and accurate estimation it is imperative that a robust estimator is employed in such circumstances.

This chapter is organized as follows: An overview of regression analysis is presented in section 5.3. In section 5.4 the concept of breakdown point is discussed. In section 5.5, available robust regression methods are reviewed. The shortcoming of the LMS estimator is presented in section 5.6. The MCS estimator is described in section 5.7, followed by the conclusion in 5.8 .

\subsection{Overview of Regression Analysis}

The main objective of regression analysis is fitting equations to observed variables. To illustrate the concept, simple linear regression examples are used throughout this chapter. The simple regression model is:

$$
\begin{aligned}
y_{1} & =x_{1} \theta_{1}+\theta_{2}+e_{1} \\
y_{2} & =x_{2} \theta_{1}+\theta_{2}+e_{2} \\
y_{3} & =x_{3} \theta_{1}+\theta_{2}+e_{3} \text { where } m>n \\
: & : \quad: \quad: \\
y_{m} & =x_{m} \theta_{1}+\theta_{2}+e_{m}
\end{aligned}
$$


Where $y_{i}$ are the observation or the measurements, and $x_{i}$ are the explanatory variable, or the index of the observations. The main objective in regression analysis, as opposed to state estimation, is to estimate the regression coefficients $\theta_{i}$ (unknowns):

$$
\hat{\theta}=\left[\begin{array}{l}
\hat{\theta}_{1} \\
\hat{\theta}_{2}
\end{array}\right]
$$

which makes it possible to obtain estimated measurements $\hat{y}$, which can be calculated as:

$$
\hat{y}_{i}=x_{i} \hat{\theta}_{1}+\hat{\theta}_{2}+e_{i}
$$

The well-known LS regression estimator proceeds with finding the best fit of $y_{t}$ and $x_{t}$, by minimizing the sum of squared residuals between the actual and the estimated measurements.

$$
\operatorname{Min}_{\hat{\theta}} \sum_{i=1}^{m}\left(y_{i}-\hat{y}_{i}\right)^{2}
$$

Figure 5.1 illustrates the LS fit, showing the scatter plot of seven points, $\left(x_{1}, y_{1}\right), \ldots \ldots$, $\left(x_{7}, y_{7}\right)$, which almost lie on a straight line. As can be seen from the figure, the LS estimator seems to have perfectly fitted all of the measurements. Let us now assume that an error takes place in the communication or processing of measurements, affecting (for instance) the decimal point positioning of the values of $x_{6}$ and $x_{7}$. As a result these points lie away from their ideal position (i.e. ideal position being indicated by diamond points in the scatter plot). These two points are an example of outliers in the $x$-direction. Figure 5.2 illustrates that scenario. It is apparent that the LS estimator has difficulties 
fitting the good measurements, and the outliers have a great influence on the LS solution, which differs from the solution in figure 5.1.

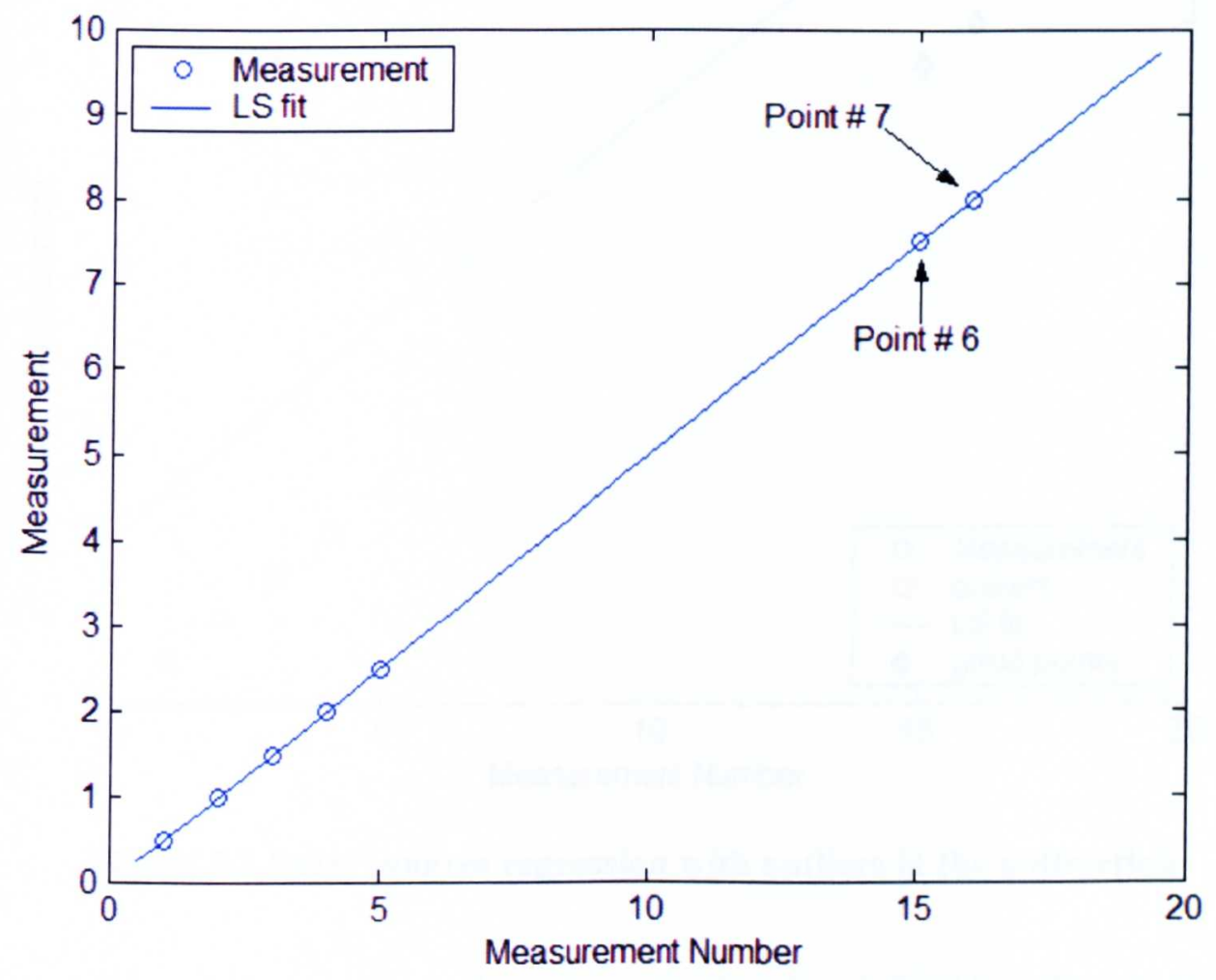

Figure 5.1 Least squares regression with no outliers

There would be similar problem for outliers occurring in the $y$-direction. Figure 5.3 illustrates such a situation, where outliers in the y-direction significantly influence the regression coefficients of the LS estimator. The points $\left(x_{6}, y_{6}\right)$ and $\left(x_{7}, y_{7}\right)$ actually tilt or "pull" the line of the LS fit. With the terminology borrowed from mechanics, theses points are called Leverage Points. In general, a leverage point is an observation that is isolated from the bulk of the observations. 


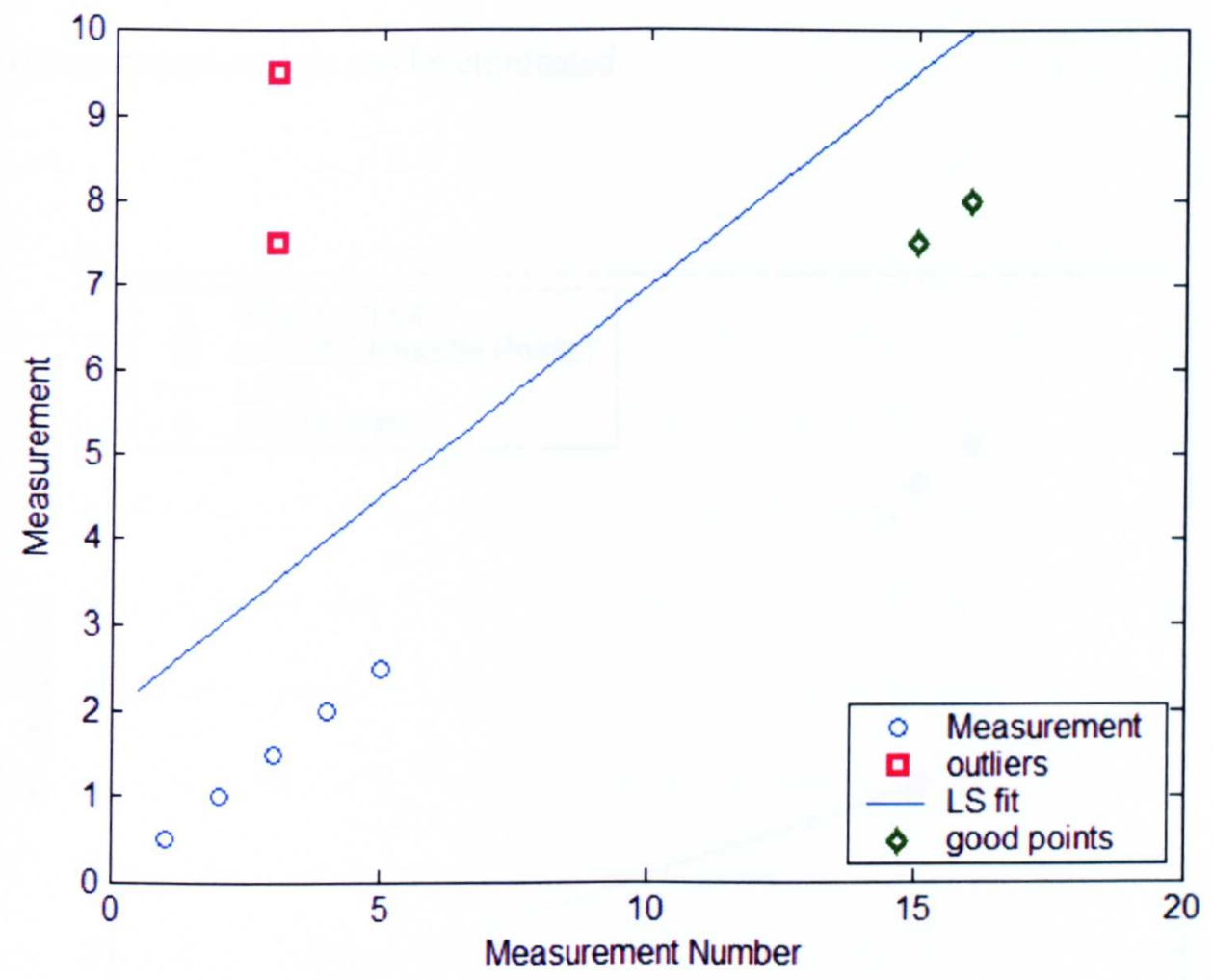

Figure 5.2 Least squares regression with outliers in the $x$-direction

It is a point that deviates severely from the regression line defined by other observations. Normally, leverage measurements can appear in power system networks in areas of low local redundancy $[80,85]$.

Other causes of leverage measurements in power systems are claimed to be $[80,85,86]$ :

- Injection measurements taken at a bus with a large number of incident branches.

- Flow measurement on a line with impedance that is drastically different to most other lines in the system. 
Abur et. al presented in [86], a 'matrix stretching' technique, by which conditions leading to leverage measurements can be eliminated.

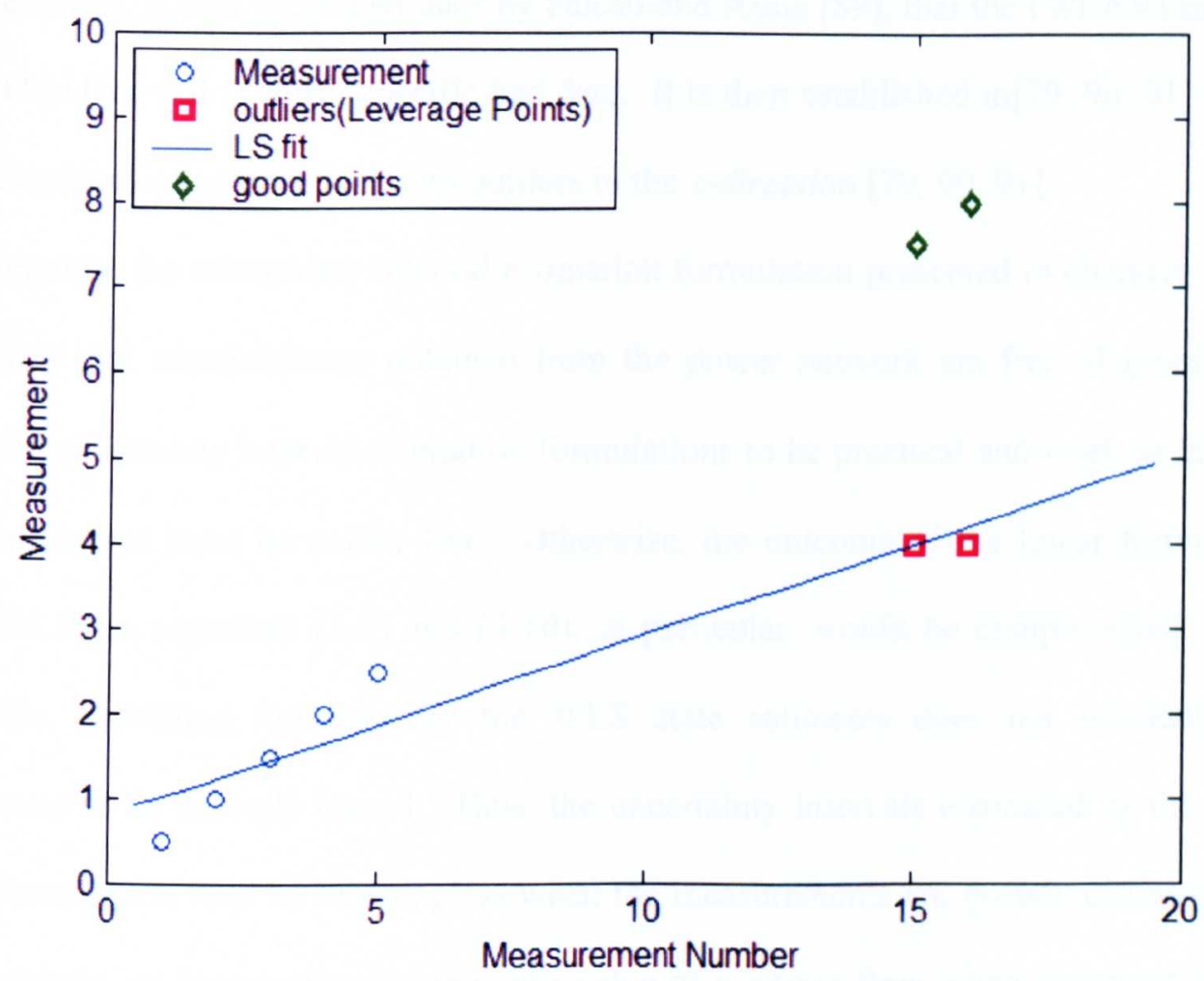

Figure 5.3 Least squares regression with outliers in the direction

The difficulties posed by the LS estimator can be attributed to the non-robust nature of least squares fit. As an attempt towards a more robust estimator, the least absolute value (LAV) regression estimator was proposed by Edgeworth [87]. The objective is to minimize the least absolute value of the residual:

$$
\operatorname{Min}_{\hat{\theta}} \sum_{i=1}^{m}\left|y_{i}-\hat{y}_{i}\right|
$$


The weight least absolute value (WLAV) estimator was considered first by Irving, Owen and Sterling [12] as a robust estimator. Confirming the robustness, Kotiuga and Vidyasagar have proved the bad data rejection is a property of (WLAV) estimator [88]. Nonetheless, it was illustrated later by Falcao and Assis [89], that the (WLAV) estimator would indeed fail to reject specific bad data. It is then established in $[79,90,91]$ that the LAV estimator can only deal with outliers in the $x$-direction $[79,90,91]$.

Regrettably, the uncertainty interval estimation formulation presented in chapters 3 and 4 assumes that measurements obtained from the power network are free of gross errors. For the uncertainty interval estimation formulations to be practical and work as intended, measurements must be outlier free. Otherwise, the outcome of the linear formulations (WLS-LP) in equations (3-9) and (3-10), in particular, would be compromised. To be specific, providing intervals for the WLS state estimates does not preclude these estimates to be strongly biased. Thus, the uncertainty intervals estimated by the (WLSLP) formulation may be meaningless when the measurements are grossly erroneous. As an example, an incorrectly metered zero value of a power flow when its actual value is within the upper and lower measurement limits.

This weakness of the previously provided formulation for estimating the uncertainty interval may be overcome by using a static robust estimator instead of the WLS, such as Least Median Squares (LMS) or Least Trimmed Squares (LTS) [78, 79, 92]. Generally, robust estimators are those estimators that exhibit stable behaviour (bounded bias and variance) under deviation from the assumptions on which they are based. That way, any outliers would be identified and eliminated from the measurement set, prior to the 
estimation process for the uncertainty bounds. Consequently, the outcome of the proposed formulations would be more precise and meaningful.

Before overviewing the robust regression methods described in the literature, it is essential to define how one can measure the robustness of a given regression procedure or robust estimator.

\subsection{Breakdown Points}

Several concepts have been proposed in the literature on how to analyse the stability of an estimator. Hampel proposed the concept of an influence function, in [93], in order to assess the robustness of an estimator. Hodges, in [94], has introduced another way to quantify the robustness of an estimator used in regression analysis. It was called the Breakdown Point. Nonetheless, Hodges definition was limited to the one-dimensional estimation of location. Hampel provided a more generalized definition for the breakdown point in [95]. A simple version and a widely used definition of the breakdown point was introduced by Donoho and Huber [96], which can be loosely defined as the smallest fraction of contaminations that critically offsets the estimator from the true measurements. Formally, the breakdown point of an estimator can be defined for a set of $m$ measurements as follows: Let

$$
Z=\left\{\left(x_{11}, \ldots, x_{1 n}, y_{1}\right), \ldots .,\left(x_{m 1}, \ldots, x_{m n}, y_{m}\right)\right\}
$$

also, let $T$ be the regression estimator being evaluated for robustness. Then, applying $T$ to the set of samples $Z$, would yield the (estimated) regression parameter as 


$$
T(Z)=\hat{\theta}
$$

Let the contaminated sample $Z^{\prime}$, which is obtained by replacing $p$ number of measurements in $m$ by erroneous values. Then the maximum bias as a result of such contamination [79] is

$$
\operatorname{bias}(p ; T, Z)=\sup _{Z^{\prime}}\left\|T\left(Z^{\prime}\right)-T(Z)\right\|
$$

where the supremum is over all possible $Z^{\prime}$, (the supremum is the least upper bound over the set $\left.Z^{\prime},[97]\right)$. If we assume that the $\operatorname{bias}(p ; T, Z)$ is finite, then that leads to $p$ outliers can have serious effect on the estimator $T$. Hence, the breakdown point of the estimator is

$$
\varepsilon_{n}^{\cdot}(T, Z)=\min \left\{\frac{p}{m} ; \operatorname{bias}(p ; T, Z) \text { is finite }\right\}
$$

Even though it was illustrated in figures 5.2 and 5.3 , that the LS estimator has failed with two outliers in the measurement set; Rousseeuw and Leroy [79] have shown examples with the least squares estimator failing with only a single outlier in the measurement set. Therefore, the breakdown point for the LS estimator is

$$
\varepsilon_{n}^{*}(T, Z)=\frac{1}{m}
$$

If the number of observation, $m$, increases, then the least squares estimator has a breakdown point of $0 \%$. That breakdown percentage clearly shows how vulnerable the least squares estimator is to outliers. The LAV estimator also has the same breakdown point, since it can fail with one leverage point as shown in [79]. 


\subsection{Overview of robust regression}

Robust estimators are those estimators that are resistant against all kinds of outlier and leverage points. Generally, robust estimators can be classified according to two main categories:

- Robust regression methods.

- Regression diagnostic methods.

Robust regression methods and regression diagnostic methods have the same objective, but proceed in the opposite order in the manner by which they accomplish that objective. Both classifications are briefly explained in the following subsections.

\subsubsection{Robust regression methods}

The robust regression approach starts the estimation procedure by fitting the bulk (the majority) of the data and at the same time reveals the outliers as observations with large residuals.

Generally, one would like to have a robust procedure that has a very high breakdown point. In theory, however, the highest breakdown point one can achieve is 0.5 (or $50 \%$ ) because for any higher contamination level, one is not guaranteed to be able to distinguish the good points from the bad. 
To illustrate this point, let us assume that one is looking for a straight line fit of a given data set. Then, if the contaminating points "conspire" to form another straight line, and if the contamination level is larger than $50 \%$, one cannot distinguish between the true line and the line represented by the contamination.

Robust regression methods include:

- The $M$-estimator and the Generalized $M$-estimator (GM) [98], where $M$ stands for maximum likelihood. These methods are probably the most popular. They proceed to downweigh the highly influential points as well as large residual points by replacing the $\left(y_{i}-\hat{y}_{1}\right)^{2}$ in equation $5-4$, by some other symmetric function of the residual. Normally, both methods have a $30 \%$ breakdown point.

- $L$-estimator is based on linear combination of order statistics [99].

- $R$-estimator is based on the ranks of the residuals [100].

- $S$-estimator is based on the minimization of a robust $M$-scale, (estimate), of the residual scale [101-103].

- Non-quadratic estimators such as the LAV are used for bad data suppression in [104]. 
- Least Median of Squares (LMS) and Least Trimmed Squares (LTS) estimator approaches $[78,79,105-107]$. They are both based on minimizing a robust measure of the scatter of the residual.

In this chapter, the LMS estimator is considered amongst the above robust methods to demonstrate the concept of robust regression and for comparison with the MCS method proposed in this thesis.

\subsubsection{Least Median of Squares}

The idea of minimizing the mean of absolute (or squared) residuals was first introduced by Hampel in [108]. Rousseeuw developed the least median of squares estimator (LMS) [78]. The LMS estimator is based on the following objective function:

$$
\operatorname{Min}_{\hat{\theta}} \operatorname{median}\left[\left(y_{1}-\hat{y}_{1}\right)^{2}\right]
$$

where $\hat{y}_{i}=\hat{\theta}_{1} x_{i}+\hat{\theta}_{2}$, for the simple regression case. The LMS estimator is obtained by minimizing the $h$ th-order residual, where $h=[m / 2]+[(n+1) / 2]$ [79]. Mili et. al [81] have derived a general expression for the optimal order, such that the breakdown point of the LMS attains the highest possible fraction of outliers. 


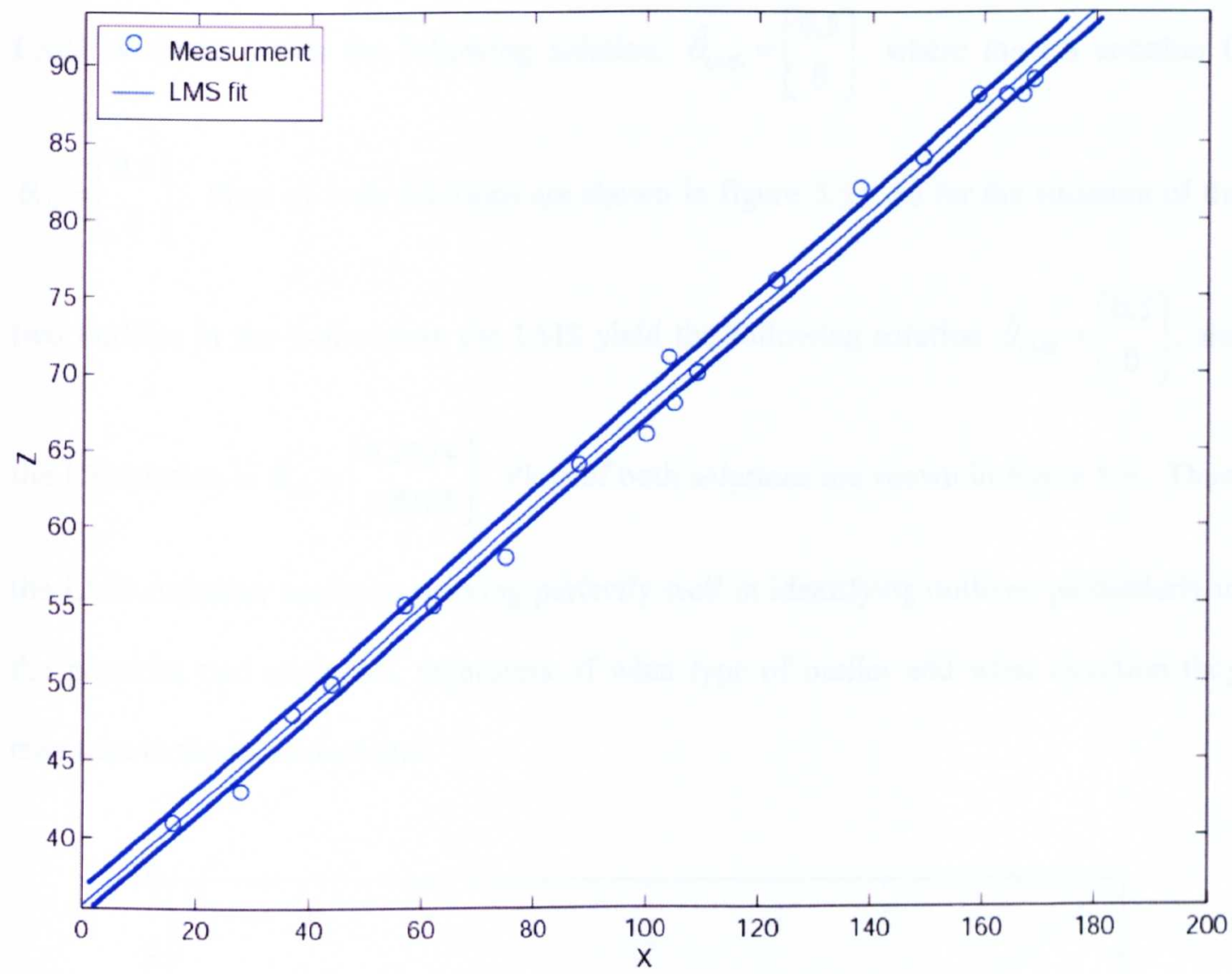

Figure 5.4 The LMS strip covering half of the measurements.

The LMS objective function can be geometrically explained as finding the narrowest strip covering half of the measurements, i.e. $m / 2$, and the LMS line lies in the middle of that strip, as illustrated in figure 5.4. This property makes the LMS a robust estimator with $50 \%$ breakdown point. For multiple regression, the LMS is obtained from the smallest plane or hyperplane slice that covers the majority of the data.

The LMS estimator performs remarkably well on the previous example where the LS and the LAV have failed. For the situation where the two outliers are in the $x$-direction, the 
LMS estimator yields the following solution: $\hat{\theta}_{\mathrm{LMS}}=\left[\begin{array}{c}0.5 \\ 0\end{array}\right]$, where the LS solution is $\hat{\theta}_{\mathrm{LS}}=\left[\begin{array}{c}0.5 \\ 2\end{array}\right]$. Plots of both solutions are shown in figure 5.5. As for the situation of the two outliers in the $y$-direction, the LMS yield the following solution $\hat{\theta}_{\mathrm{LMS}}=\left[\begin{array}{c}0.5 \\ 0\end{array}\right]$, and the LS solution is $\hat{\theta}_{\mathrm{LS}}=\left[\begin{array}{l}0.2124 \\ 0.8185\end{array}\right]$. Plots of both solutions are shown in figure 5.6. Thus, the LMS estimator seems to working perfectly well in identifying outliers, particularly in the previous two examples, regardless of what type of outlier and what direction they may take in the measurements.

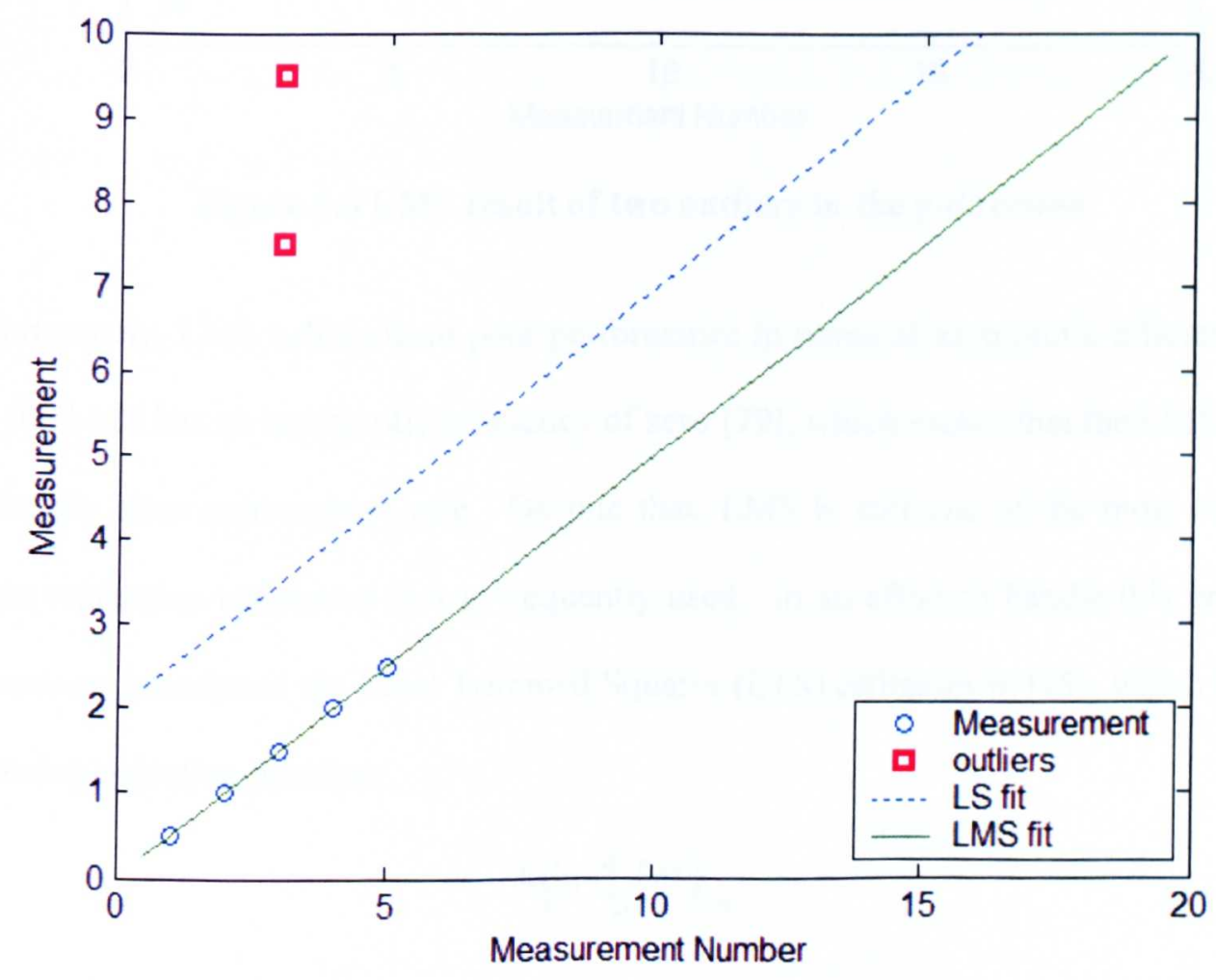

Figure 5.5 LMS result of two outliers in the $x$-direction 


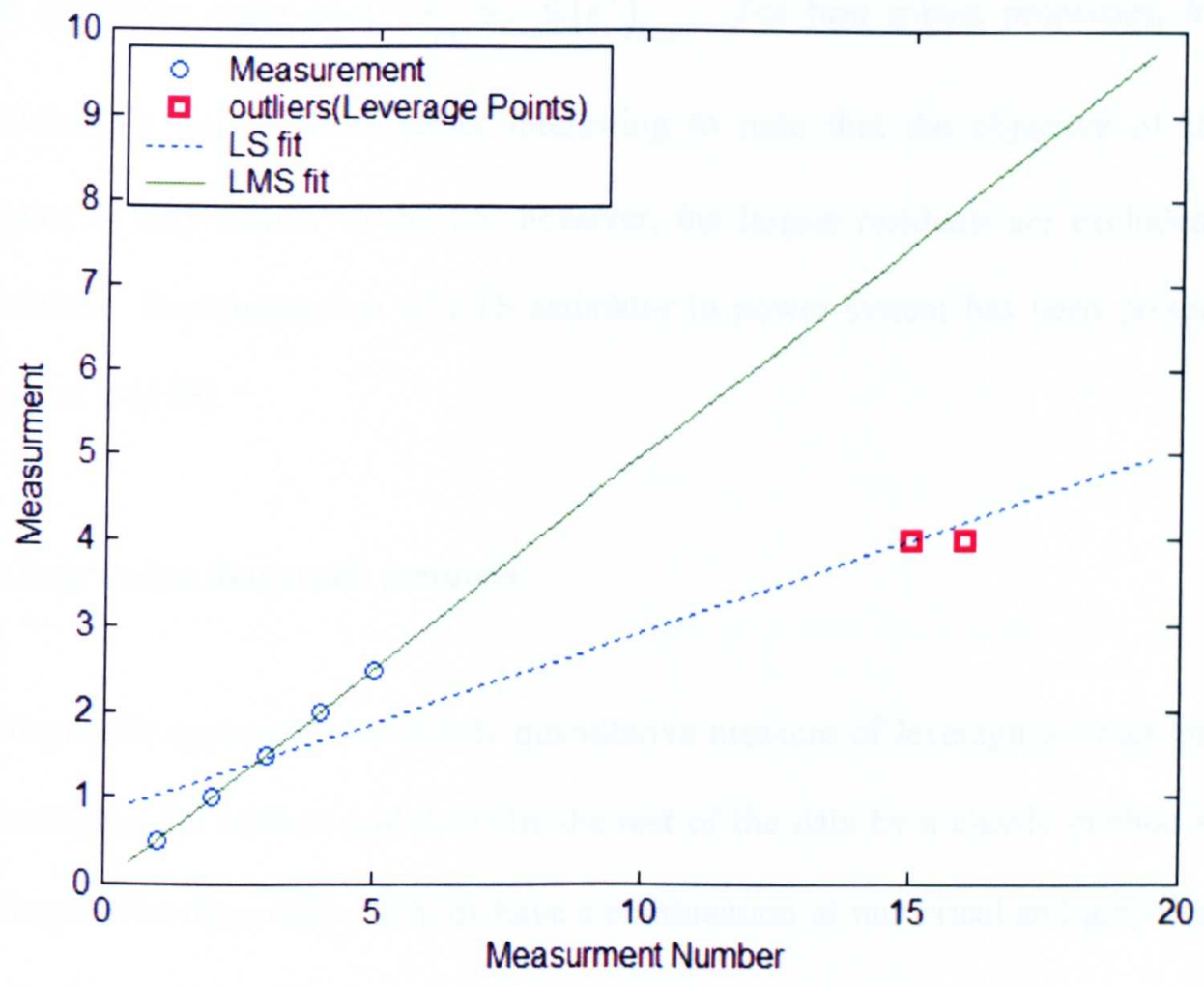

Figure 5.6 LMS result of two outliers in the y-direction

Unfortunately, LMS suffers from poor performance in terms of asymptotic efficiency. In fact the LMS has an asymptotic efficiency of zero [79], which means that the LMS has an apparently slow convergence rate. Despite that, LMS is still one of the most common robust regression estimators that is frequently used. In an effort to handle this problem, Rousseeuw introduced the Least Trimmed Squares (LTS) estimator in [79], which has the following objective function:

$$
\operatorname{Min}_{\dot{\theta}} \sum_{i=1}^{h}\left(r^{2}\right)_{i: m}
$$


where $r$ is the residual vector of all measurements. Note that the residuals are arranged in an ascending order as $\left(r^{2}\right)_{1: m} \leq \ldots \leq\left(r^{2}\right)_{m: m}$. For best robust properties, $h$ has to approximately $m / 2$. It is rather interesting to note that the objective of the LTS estimator is very similar to the LS, however, the largest residuals are excluded in the summation. Implementation of LTS estimator in power system has been presented by Mili et. al in [109].

\subsubsection{Regression diagnostic methods}

The diagnostic approach is a strictly quantitative measure of leveraging which proceeds by identifying the outliers and then fits the rest of the data by a classic method such as LS. Regression diagnostic methods have a combination of numerical and graphical tools for detection and identification of outliers.

In general, regression diagnostic methods may be categorized as:

- Classical diagnostic methods.

- Robust diagnostic methods.

Both methods are briefly explained in the following subsections.

\subsubsection{Classical diagnostic methods}

The classical method computes what is known as the Mahalanobis distance as 


$$
M D_{i}=\sqrt{\left(X_{i}-T(X)\right) C(X)^{-1}\left(X_{i}-T(X)\right)^{\prime}}
$$

for every $X_{i}$, where $T(X)$ is the arithmetic mean of the data set $X$ and $C(X)$ is the usual sample covariance matrix. For a simple regression case, the data set matrix $X$ looks like

$$
X=\left[\begin{array}{l}
X_{1} \\
X_{2} \\
X_{3} \\
X_{4} \\
X_{5}
\end{array}\right]=\left[\begin{array}{cc}
x_{1} & 1 \\
x_{2} & 1 \\
: & 1 \\
x_{m-1} & 1 \\
x_{m} & 1
\end{array}\right]
$$

The significance of that distance, $M D_{i}$, is it indicates how far a given state $x_{i}$ is from the centre of the cloud of data. Although this method proved to be useful in identifying outlying effects in measurements, unfortunately, this approach seriously suffers from the masking effect of outliers. Rousseeuw and Zomeren have argued in $[110,111]$, that multiple outliers may have some masking effects on each other and do not necessarily have $M D_{i}$ far away from the cloud. This finding seems to be rather obvious, since neither $C(X)$ or $T(X)$ are robust against outliers, in particular leverage points.

\subsubsection{Robust diagnostic methods}

In an effort to combat the masking effects, Rousseeuw and Zomeren have proposed the robust distance denoted as $(R D)$, in $[110,111]$, where $C(X)$ and $T(X)$ in equation 513 are based on minimum volume ellipsoid (MVE) proposed by Rousseeuw in [112]. The robust distance may be calculated as 


$$
R D_{i}=\sqrt{\left(X_{i}-T(X)\right) C(X)^{-1}\left(X_{i}-T(X)\right)^{\prime}}
$$

where $T(X)$ is the centre of the minimum volume ellipsoid covering half of the measurements, and $C(X)$ may be determined by the same ellipsoid multiplied by a correction factor.

Figure 5.6 illustrates the distinction between the classical and the robust diagnostic methods. The data in this example are from Hertzsprung-Russell for star clusters, which are obtained from [79].

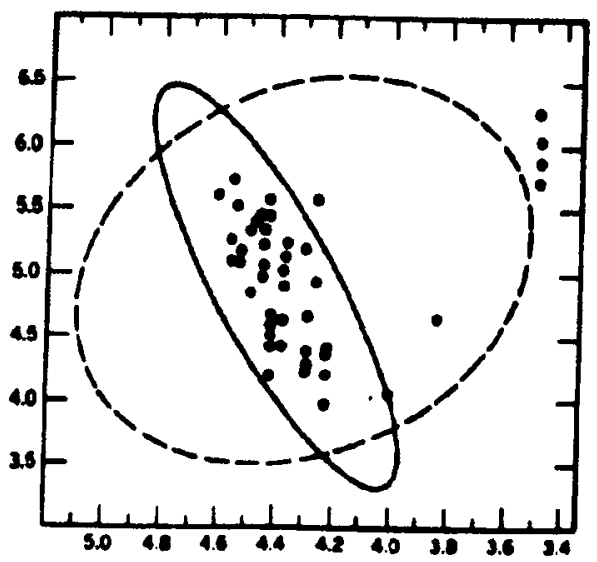

Figure 5.6 Mahalanobis distance (dashed ellipse) verses Robust distance (dashed ellipse)

The plot shows 47 points of $\log$ temperature (x-axis) versus the log of the light intensity (y-axis), with $97.5 \%$ tolerance ellipse. The dashed line ellipse is obtained form the classical mean and covariance (Mahalanobis distance), which is quite large, due to the attraction of the top right hand side four outliers. The classical ellipse is trying to engulf the outliers. On the other hand, the solid line ellipse (Robust distance) is produced based on the MVE estimator, which is smaller and essentially fits the main bulk. Therefore, the 
extreme outliers can be identified with high certainty as outliers, since they are far a way from the bulk designated by the MVE ellipse.

Robust diagnostics have been applied in power system state estimation by Mili et. al [82, 83], and were referred to as projection statistics. It was shown that projection statistics indicate the degree of leveraging in a given set of measurements.

\subsection{The shortcoming of the LMS estimator}

The LMS estimator was initially appealing due to its robustness and effectiveness in detecting outliers. However, in certain circumstances, the LMS is not very effective. One situation where sever problems can potentially occur for LMS is when collinearity exists in the measurements. Collinearity may be defined as near linear dependency among the measurements [113]. Collinearity can cause large variability in the state estimates, occasionally resulting in estimates that differ from the true values by an order of magnitude and/or have the incorrect sign.

\subsubsection{Illustrative example}

Stefanski has argued that high-breakdown estimators, e.g. LMS, can exhibit unusual finite-sample behaviour [114]. Stefanski has shown, via a simple example, that an LMS estimator may produce dubious estimates where collinearity does exist among a certain set of measurements. This simple example was slightly modified by Ryan, in [92], to further illustrate the weakness of the LMS estimator. The data for this example is shown 
in table 5.1. The outcome of LMS and LS estimators on the Stefanski example is illustrated in figure 5.8 .

Table 5.1 Ryan's example

\begin{tabular}{c|c}
\hline $\mathrm{X}$ & $\mathrm{Y}$ \\
\hline 2.5 & 5.0 \\
\hline 7.5 & 2.6 \\
\hline 14.0 & 2.6 \\
\hline 15.0 & 3.9 \\
\hline 16.0 & 5.1 \\
\hline 22.0 & 13.0 \\
\hline 23.0 & 14.2 \\
\hline 23.0 & 5.1 \\
\hline 29.0 & 2.7 \\
\hline
\end{tabular}

It appears from figure 5.8 that the outliers have successfully misled the LMS fit and attracted its line. This phenomenon is mainly caused by collinearity in the measurements. In this example $m=9$ and $n=1$, therefore the 5 lower valued squared residuals will be minimized by LMS. Interestingly, five points of the data set fall on the same line, three good points and two outliers, which consequently appears to be the best candidate solution that the can be provided by the LMS estimator. Thus, for this example, the LMS solution was very far from the correct solution. In comparison, the outliers seem to have less effect on the LS estimate. Subjectively, the LS fit appears to be better and closer to a correct solution (a correct solution should have approximately a zero slope). Certainly, had the seven good data only been used in the LMS regression procedure, the slope would have been virtually zero. However, the collinearity of three good data points with two outliers has misled the LMS estimator. 


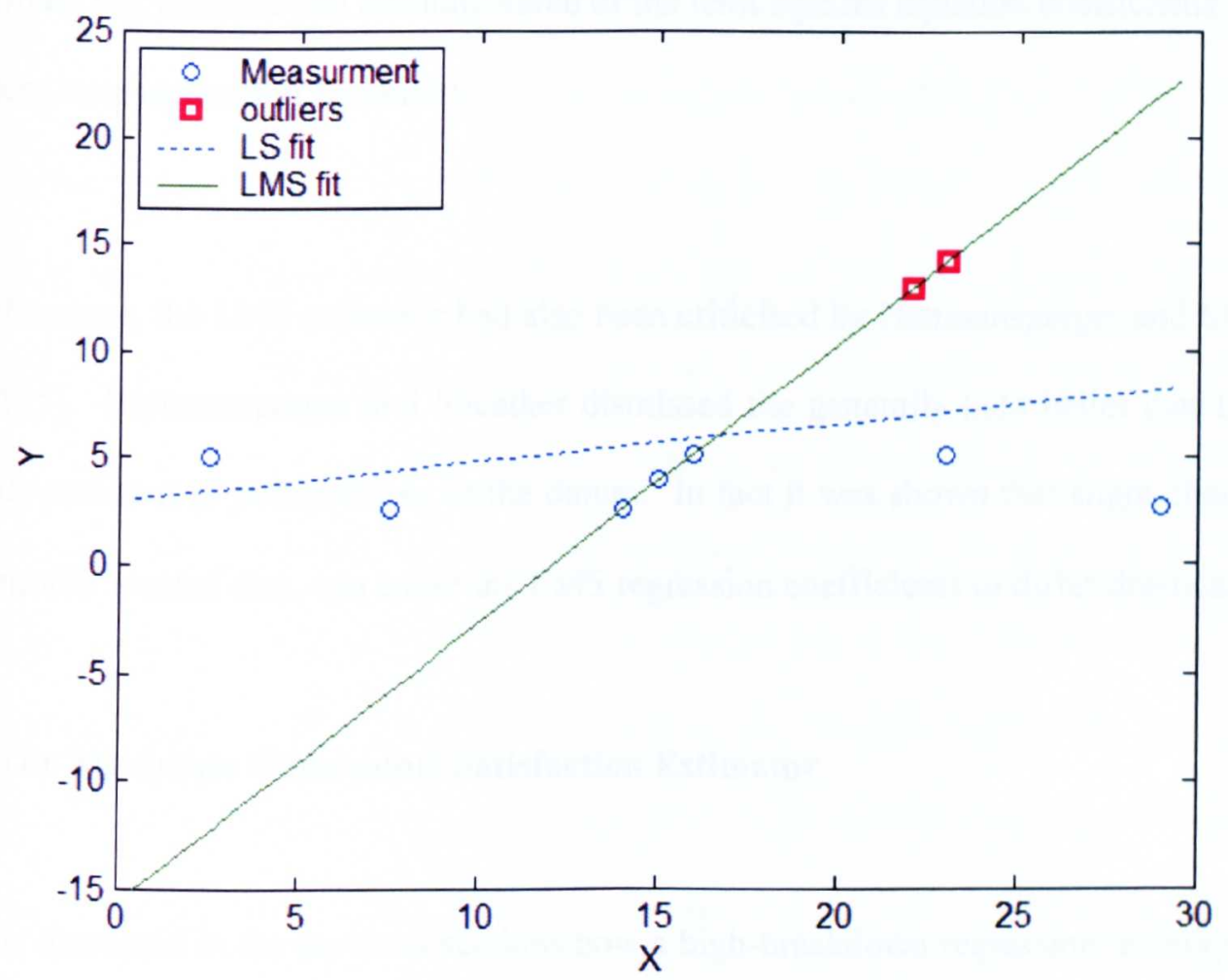

Figure 5.8 Illustration of LMS shortcoming

Collinearity of measurements in power systems state estimation takes place as a consequence of unintended dependence among measurements [84]. For instance, the measurement of frequency at two points in a power system for a power system stabilizer (PSS) application may be collinear if the same reference clock is used for both measurements. Furthermore, common mode error in measurements often results in collinearity. As an example, the use of a common sequence ground for several voltage measurements. In such a case, the measurements may be collinear. Mathematically, collinearity could very well result in an ill-conditioned matrix, meaning that the matrix 
inversion can be very inaccurate [113]. Generally, collinearity in the measurements tends to inflate the variance and absolute value of the least squares equation coefficients (power system state estimation Jacobian).

Furthermore, the LMS estimator had also been criticised by Hettmansperger and Sheather in [115]. Hettmansperger and Sheather dismissed the generally held belief that LMS is highly resistant to perturbations in the datum. In fact it was shown that slight changes in a centrally located data, can cause the LMS regression coefficients to differ drastically.

\subsection{The Maximum Constraints Satisfaction Estimator}

It was discussed in the previous sections how a high-breakdown regression technique, i.e. LMS, suffers from statistical difficulties. Authors in $[92,114,115]$ have particularly discussed many illustrative examples showing LMS estimator failing in identifying outliers. Indeed, collinearity and perturbations in the measurements generally undermine the robustness of an LMS fit.

The concept of uncertainties in the measurements may be considered to develop a more robust estimator. In this section a new robust state estimator is proposed based on measurements uncertainty. The proposed Maximum Constraints Satisfaction (MCS) estimator has the ability not only to detect outliers, but is also resistant with respect to bad leverage points. The MCS estimator is based on the idea of searching for a point $\underline{x}$ 
in the space of all possible estimates that maximizes the number of satisfied uncertain measurements. To be specific, each measurement with its associated uncertainty can be represented by double inequality constraints (upper and lower limits). These constraint limits define the tolerances on the measurements (i.e. the range of values within which the true value of the measured quantity must lie). A point $\underline{x}$ satisfying most of the available double inequality constraint, if not all, must be a valid solution point. The fundamental concept of the MCS estimator may be explained better by the illustration in figure 5.9. This illustration shows three uncertain measurements along with their bounds. The intersection of these bounds defines an area known as the "feasible region" of the uncertain measurements. An arbitrary point in the feasible region may certainly be considered as a potential solution that satisfies all those uncertain measurements (i.e. double inequality constraints). It must be noted that any given solution point in the feasible region is not necessarily optimum but is a valid feasible solution.

Suppose that outliers exist in the measurement set. These outliers with their bounds might, or might not, create a region of their own. If by coincidence the uncertain bounds of the erroneous measurements mange to establish a region of their own, a solution point in that region can never have a maximum number of satisfied constraints that exceeds that of the region established by the good measurements, (given that the level of contamination in a given set of measurements can never be more than $50 \%$, for the worst case scenario). Therefore, the MCS estimator guarantees a robust solution, which is not influenced by outliers or erroneous measurements. That is because the MCS estimator always seeks a solution point in the feasible region with the largest number of satisfied 
measurements, (which ought to be created only by the good measurements in the set). Consequently, erroneous measurements can never affect the quality of the MCS solution.

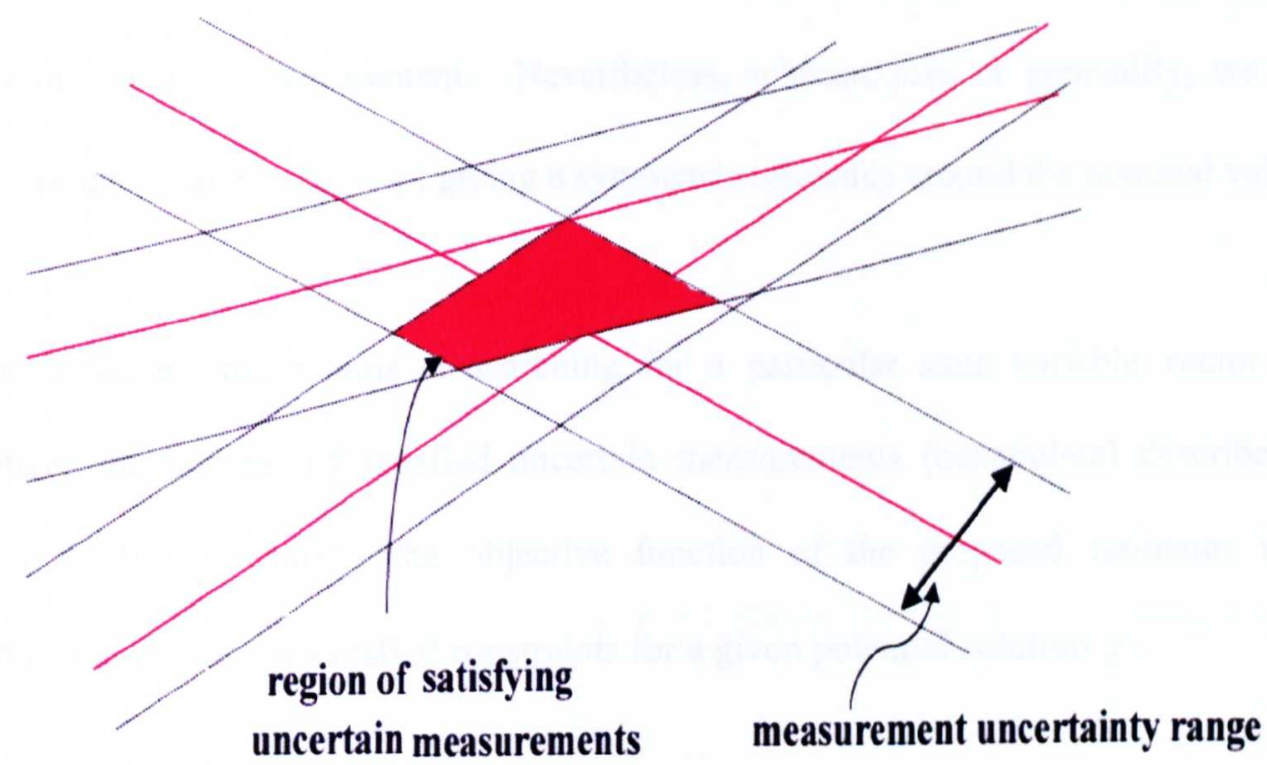

Figure 5.9 The Maximum Constraints Satisfaction

Mathematically, for a vector of measurements $\underline{z}$, the uncertain measurements may be represented by the following double inequality constraints:

$$
\begin{gathered}
z_{1}^{l} \leq h(\underline{x}) \leq z_{1}^{u} \\
\vdots \quad \vdots \quad \vdots \\
z_{m}^{l} \leq h(\underline{x}) \leq z_{m}^{u}
\end{gathered}
$$

where $h(\underline{x})$ is set of mathematical equation that relates the states $\underline{x}$ to the measurements $\underline{z}$. Also $\underline{z}^{\prime}$ is the lower bound of the measurement vector and $\underline{z}^{u}$ is upper bound, and they are formed as: 


$$
\begin{aligned}
& \underline{z}^{\prime}=\underline{z}-\underline{\tau}^{-} \\
& \underline{z}^{u}=\underline{z}+\underline{\tau}^{+}
\end{aligned}
$$

where $\underline{\tau}^{+}$and $\underline{\tau}^{-}$are the transducer tolerances. The tolerances describe the deterministic uncertainty of each measurement. Nevertheless, without loss of generality, we will usually assume that $\underline{\tau}^{+}=\underline{\tau}^{-}=\underline{\tau}$, giving a symmetric tolerance around the nominal value.

The proposed estimator aims at searching for a particular state variable vector that maximizes the number of satisfied uncertain measurements (constraints) described in equation (5-16). Formally the objective function of the proposed estimator is to maximize the number of satisfied constraints for a given potential solution $\underline{x}$.

\section{$\operatorname{Max} \Omega$}

where $\Omega$ denotes the number of double inequality constraints of equation 5-16 satisfied by $\underline{x}$. Geometrically, the shape of the objective function may be analogous to a campus map, where the objective is to search for the top of the tallest building. The value $\Omega$ is analogous to the number of floors in each building, and $\underline{x}$ represents the ground coordinates of a point on the campus. The tallest top is the feasible region established by the good measurements. For that reason, a Real-Coded Genetic Algorithm is chosen to solve the optimisation problem of equation $5-19$, which starts by generating a random population of potential solutions, of which each potential solution is evaluated and checked for how many double inequality constraints are satisfied. The potential solution 
with highest number of satisfied constraints, $\Omega_{i}$, is picked up by RGA for the next generation, and so on. The reason a Genetic Algorithm was chosen is because of its capability to search the whole space to find that region with the most satisfied constraints. The proposed method has been tested on simple regression test cases, a three-bus D.C test system and a six-bust A.C network. The proposed method proved to be robust and successful in identifying outliers.

\subsubsection{Simple linear regression example}

For a simple regression test case, with $m$ measurements, the MCS formulation may be given as:

$$
\begin{gathered}
\underset{\substack{\theta_{1}, \theta_{2} \\
z_{1}^{-} \leq x_{11} \theta_{1}}}{ } \Omega \theta_{2} \leq z_{1}^{+} \\
\vdots \quad \vdots \quad \vdots \quad \vdots \quad \text { where } m \succ 2 \\
z_{m}^{-} \leq x_{m 1} \theta_{1}+\theta_{2} \leq z_{m}^{+}
\end{gathered}
$$

We can now apply the MCS estimator on the example, which had two outliers in the $x$ direction from section 5.3. It was demonstrated in section 5.5.1.1, that the LMS estimator has successfully detected these two outliers. With a symmetric tolerance of, $\tau= \pm 3 \%$, applied on all seven measurements of that example, the MCS formulation would be 


$$
\begin{gathered}
\operatorname{Max}_{\theta_{1}, \theta_{2}} \Omega \\
z_{1}^{-} \leq x_{1} \theta_{1}+\theta_{2} \leq z_{1}^{+} \\
\vdots \quad \vdots \quad \vdots \quad \vdots \\
z_{7}^{-} \leq x_{7} \theta_{1}+\theta_{2} \leq z_{7}^{+}
\end{gathered}
$$

As shown in figure 5.10, the MCS has effectively solved for the correct regression coefficients, $\hat{\theta}_{\mathrm{MCs}}=\left[\begin{array}{c}0.5 \\ 0\end{array}\right]$, fitting all five good points, perfectly unaffected by the outliers in the $x$-direction. The least squares fit is shown for comparison. The bottom subfigure in figure 5.10, illustrates the number of satisfied constraints, $\Omega$, progressively increasing throughout the evolution process of GA. It is interesting to see that the maximum number of constraints that could possibly be satisfied is five, in this case, which is the number of good measurements. 

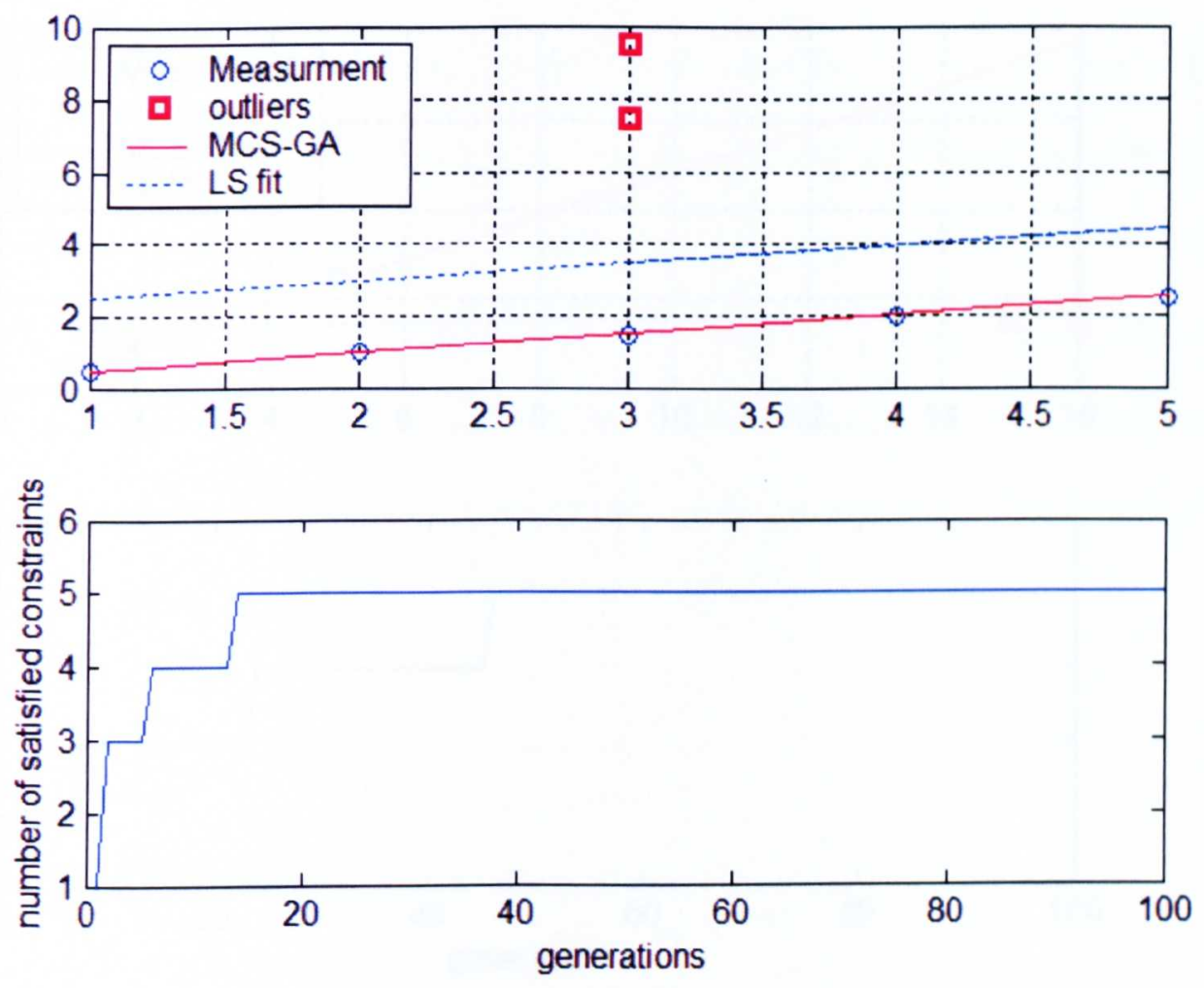

Figure 5.10 Application of MCS with outliers in the $x$-direction

As expected, when the MCS estimator has been applied to a simple regression case which had two outliers in the $y$-direction, both leverage points, the regression parameters were $\hat{\theta}_{\mathrm{MCS}}=\left[\begin{array}{c}0.5 \\ 0\end{array}\right]$. The outcome of the MCS is compared with least squares, and an illustration of that outcome is shown in figure 5.11. Clearly, these two leverage points had no effect on the MCS estimator. (For these tests, the population size $=100$, crossover $=0.8$, mutation $=0.09$ and elitism rate $=8 \%$.) 

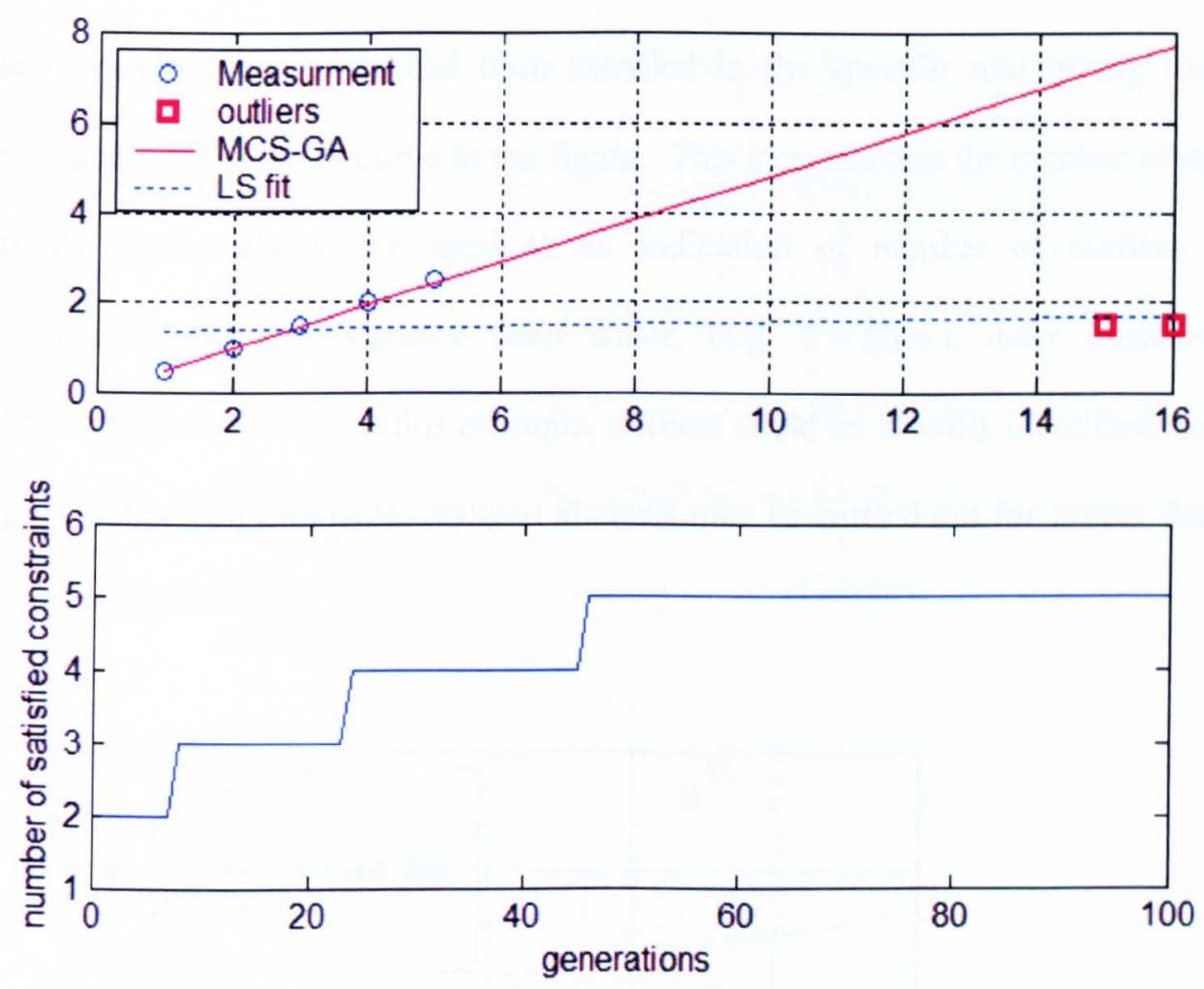

Figure 5.11 Application of MCS with outliers in the $y$-direction

So far the proposed method has effectively solved two problem, one with outliers in the $x$-direction and another with leverage points. For further assessment of the robustness and performance of the MCS estimator, particularly in situations where collinearity exists, the MCS estimator has also been tested on the data set provided by Stefanski [114] and shown in table 5.1. (With tolerance $\tau= \pm 3 \%$, population size $=100$, crossover $=0.8$, mutation $=0.09$ and elitism rate $=8 \%$.) Figure 5.12 illustrates the outcome of the MCS estimator on Stefanski's example. Apparently, the solution from that run has produced a zero slope approximately, to be specific $\hat{\theta}_{\mathrm{MCS}}=\left[\begin{array}{c}-0.0017 \\ 3.4088\end{array}\right]$. As far as the maximum 
number of satisfied measurements is concerned, it is apparent form the plot that only four double inequality constraints had been satisfied in the specific run; giving four data points adjacent to the MCS curve in the figure. This suggests that the number of satisfied constraints cannot always be used as an indication of number of outliers in the measurements. Had the tolerance been wider, (e.g. $\tau= \pm 5 \%$ ), more measurements would have been satisfied. In this example, outliers could be visually identified, however for higher dimension problems, residual analysis may be carried out for proper detection of the number of outliers.
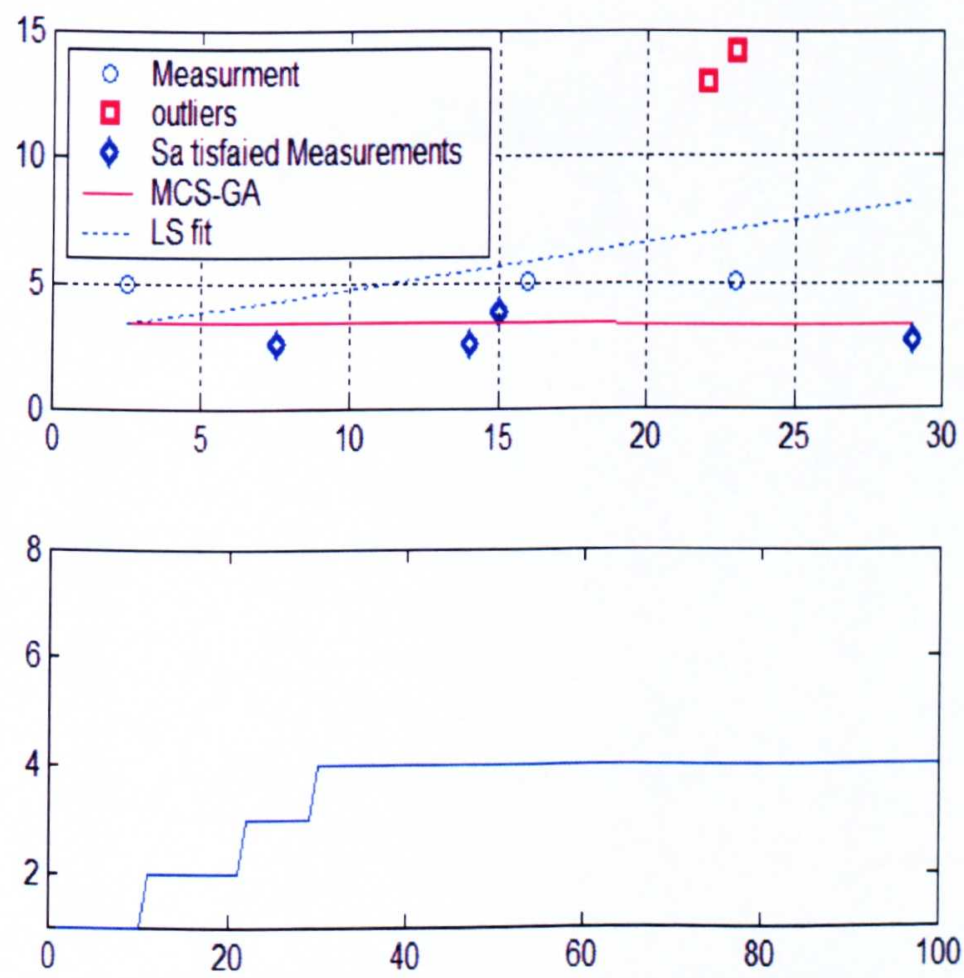

Figure 5.12 Application of MCS on Stefanski's example 
Even though the solution of the MCS seemed intuitively correct, for comparison the LS solution of measurement set, excluding the outliers, had been carried out. With the two outliers taken out form the data set, the LS regression coefficients are $\hat{\theta}_{\mathrm{LS}}=\left[\begin{array}{r}-0.0182 \\ 4.1350\end{array}\right]$. This solution is depicted in figure 5.13. As shown in the figure, the two estimators seem to have almost converged to the same regression parameters. The numerically discrepancy between the two outcomes may be attributed to the tolerance imposed on the measurements, as dictated by the formulation of the MCS estimator of uncertain measurements.

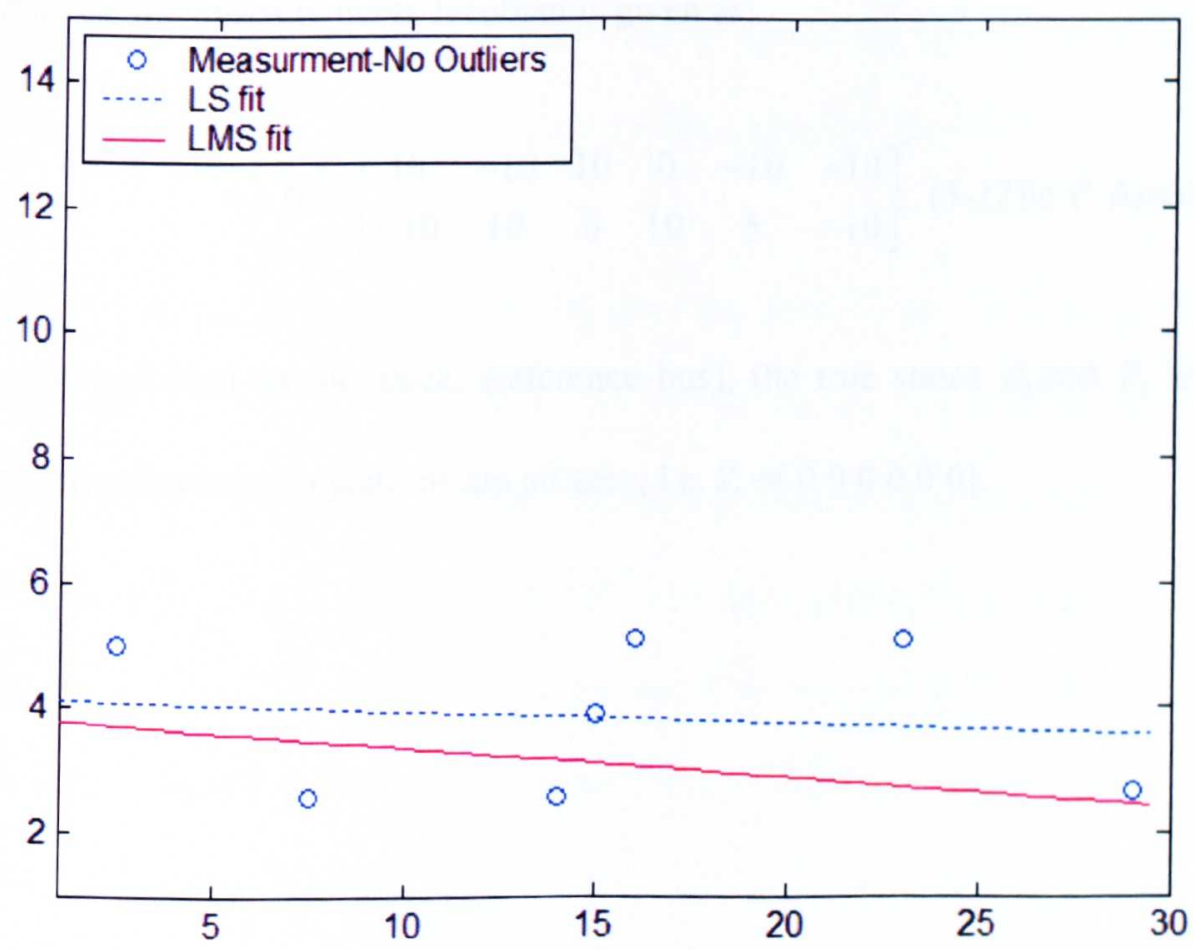

Figure 5.13 Stefanski's example without outliers 


\subsubsection{D.C. three-bus test system}

For further assessment and testing of the MCS estimator on a more realistic example, the D.C. model of a three-bus system is considered. An on-line diagram of the test system is displayed in figure 5.14. The system was originally proposed by Monticelli and et. al, in [116], in their study of the identification of multiple bad data for state estimation. Milli et. al, in [80], have applied the LMS estimator on the same system. Physically, the resistance of all lines of the three-bus network are taken to be zero. All line reactances are however to 0.1 p.u. Suppose that the six real power measurements are taken with zero variances, the measurements Jacobian is given as:

$$
H=\left[\begin{array}{cccccc}
10 & -10 & 10 & 0 & -10 & -10 \\
-10 & 10 & 0 & 10 & 5 & -10
\end{array}\right]^{T}(5-22)|\mathrm{c}|^{*} \text { Arabic } ।^{*} \text { MERGEFOF }
$$

Having assumed bus 1 as the slack, (reference bus), the true states $\theta_{2}$ and $\theta_{3}$ are zero. Hence, the true flows and injections are all zero, i.e. $Z=\left[\begin{array}{llllll}0 & 0 & 0 & 0 & 0 & 0\end{array}\right]$. 


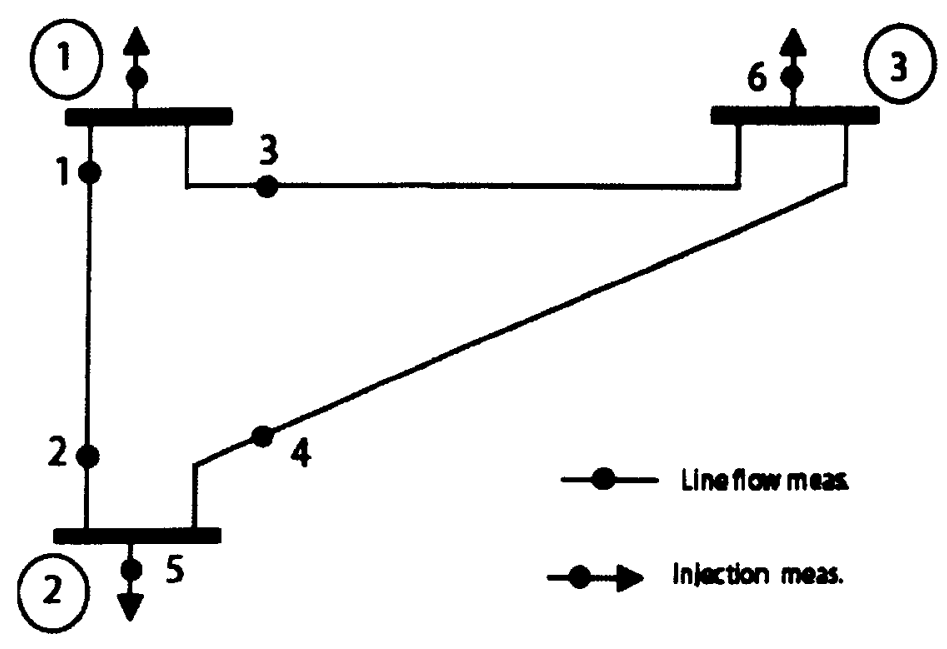

Figure 5.14 Three-bus test system

In order to test the MCS estimator, various scenarios of bad data occurring in the measurement set will be considered. In order to check the validity of the proposed method, these scenarios are exactly the same as those considered in [116].

Case A: Suppose that a single bad data had been acquired for the fifth measurement, the real power injection at bus 2, for example $Z=\left[\begin{array}{llllll}0 & 0 & 0 & 0 & 1 & 0\end{array}\right]$. The MCS estimator yields $\theta_{2}=0.0010$ and $\theta_{3}=0.0008$. From that solution the residual is calculated as $[-0.0027$, $0.0027,-0.0102,-0.0075,1.0065,0.0177]$, and the corresponding standard deviation for the residuals is 0.4110 . It is apparent that the fifth residual is the only one which is larger than the standard deviation, suggesting that the fifth measurement must be a bad data point. 


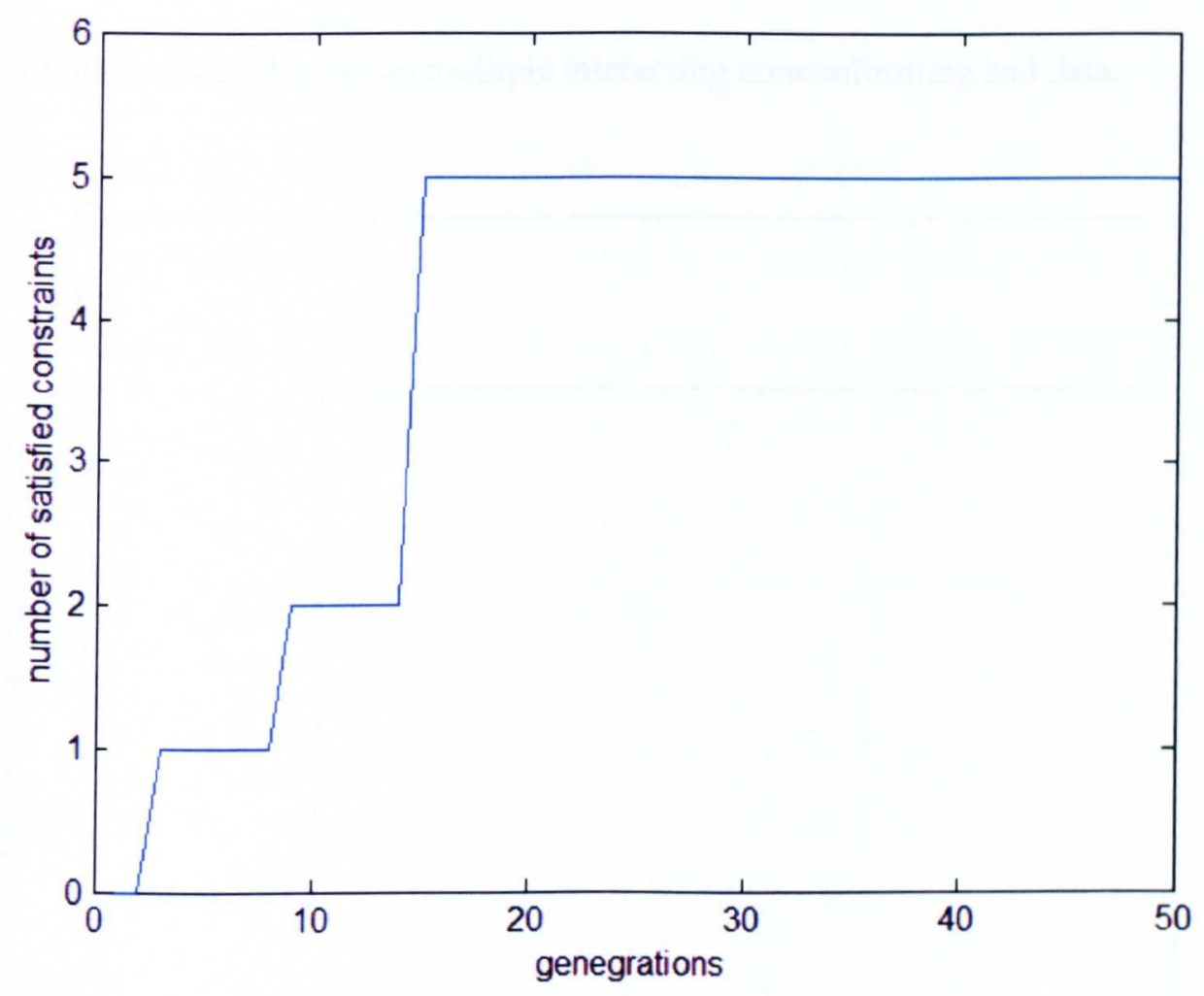

Figure 5.15 MCS performance with single bad data

Figure 5.15, displays the convergence of the MCS estimator. It shows that only five double inequality constraints can possibly be satisfied in this case, signifying that one measurement in the set must be erroneous.

Case B: Assume that readings for the same network are $Z=\left[\begin{array}{llllll}0 & -1 & 0 & 0 & 1 & 0\end{array}\right]$. Generally, measurements 2 and 5 are considered interacting, since they are both related to the line flows of bus2. These measurement values are however nonconforming. The MCS estimator yields $\theta_{2}=-0.0039$ and $\theta_{3}=0.0132$, leading to residual vector $[-0.0274$, 0.9726, $-0.0129,0.0145,1.021,-0.0016]$ with a standard deviation 0.6305 . From this 
result, it can be deduced that measurement 2 and 5 are erroneous. This type of error distribution is usually known as multiple interacting nonconforming bad data.

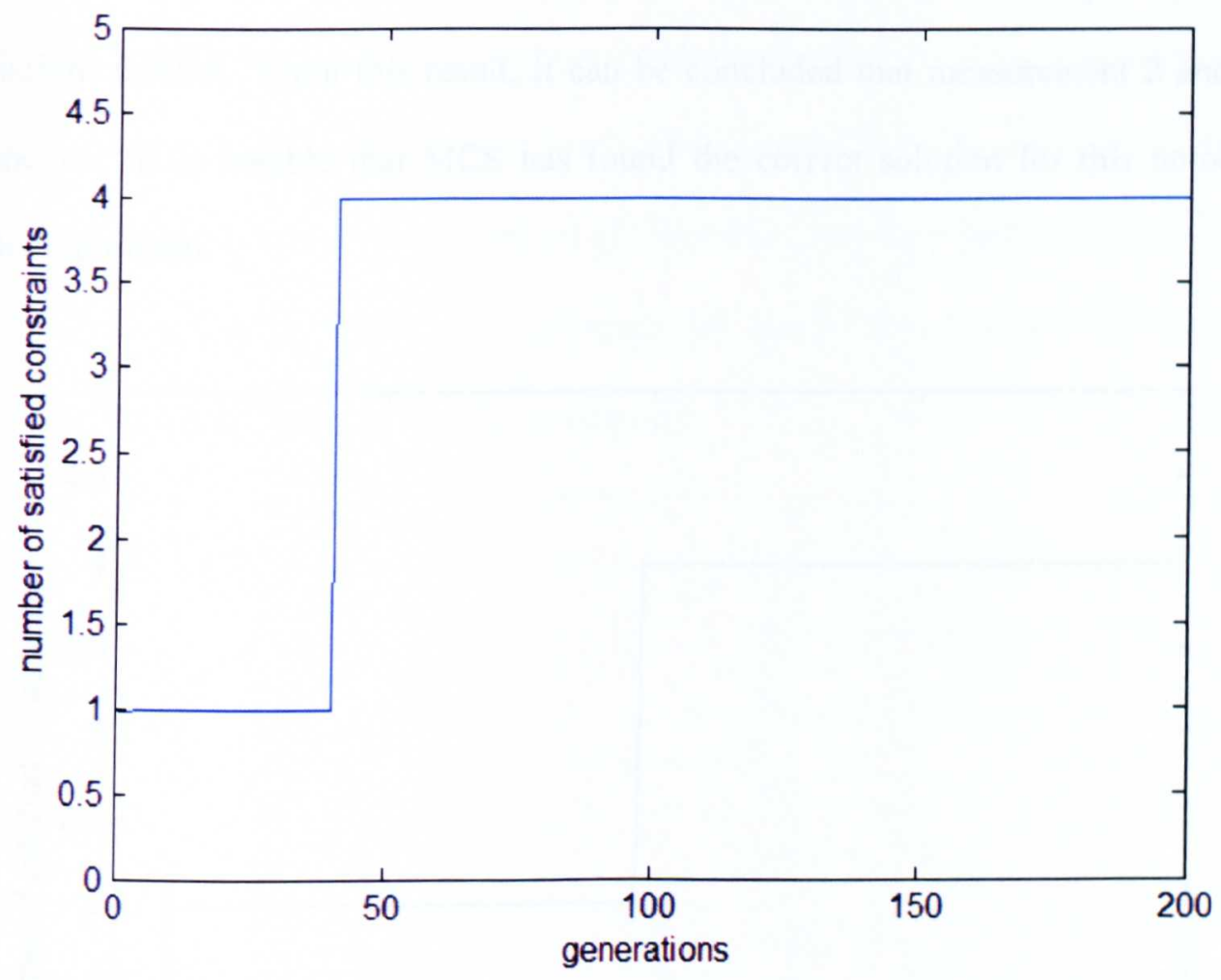

Figure 5.16 MCS performance with multiple interacting bad data

The maximum number of constraints that may be satisfied is four, as indicated by figure 5.16. That indication appears to be correct, since it is known that two out of the six readings are bad.

Case C: Suppose that readings for the same network are $Z=\left[\begin{array}{llllll}0 & 1 & 0 & 0 & 1 & 0\end{array}\right]$. In this case measurements 2 and 5 are considered interacting and conforming, since they are both related to the line flows of bus 2 and they are consistent. This is known as multiple interacting conforming bad data, which is known to cause failure in most estimators [47, 
116], in particular the largest normalized residual (LNR) method which is presented by Monticelli et. al in [117]. The MCS yields $\theta_{2}=-0.0017$ and $\theta_{3}=0.0004$, leading to residual vector $[0.0210, \quad \mathbf{0 . 9 7 9 0}, \quad 0.0168,-0.0042, \quad \mathbf{0 . 9 8 1 1},-0.0126]$ and a standard deviation 0.5035 . From this result, it can be concluded that measurement 2 and 5 are erroneous. It is notable that MCS has found the correct solution for this notoriously difficult problem.

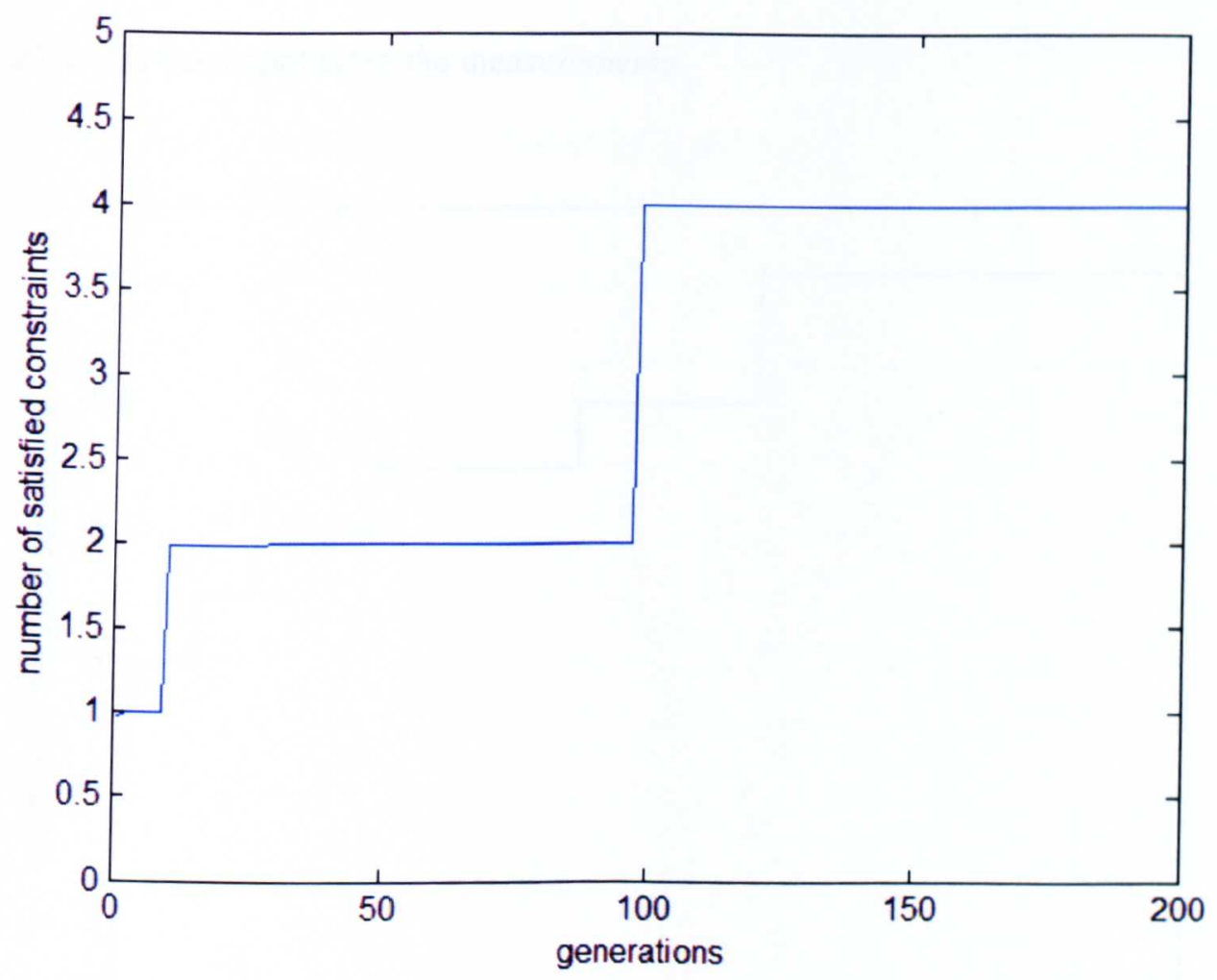

Figure 5.17 MCS performance with multiple interacting conforming bad data

Figure 5.17 shows the progressive count of satisfying constraints throughout the GA evolution. It suggests that a maximum of four constraints only can be satisfied for that 
estimation process, which is consistent with the number of good measurement in this specific example.

\subsubsection{Six bus A.C. example}

In this section the MCS estimator has been applied on an A.C six-bus network from [41]. Initially, a clean set of measurements has been fed in to the MCS estimator, including a total of twelve measurements (six real power injections and six reactive power injections, this gives a redundancy $\approx 1.1$ ). With a population size of 200 , a symmetric tolerance of $\tau= \pm 5 \%$ has been applied to the measurements.

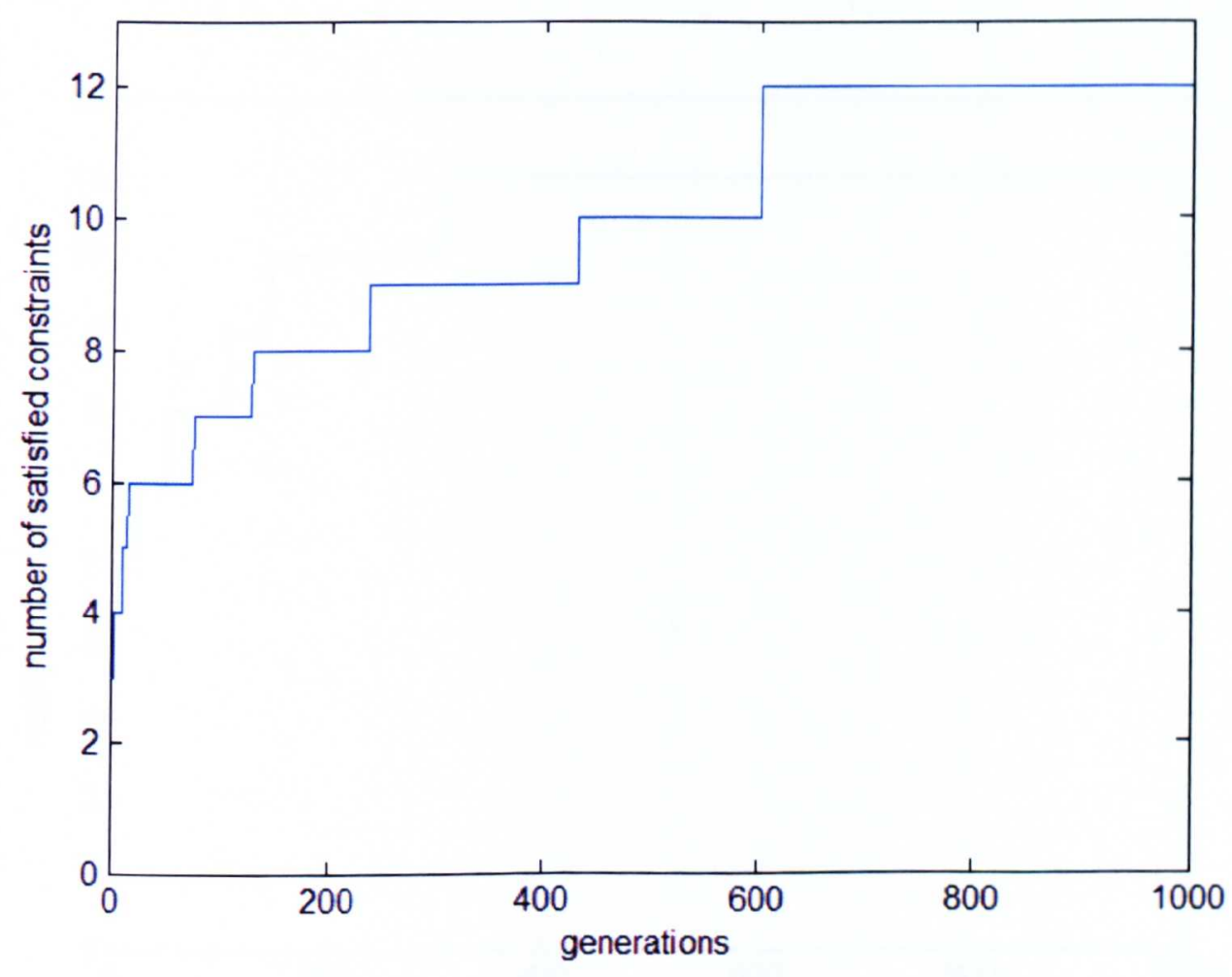

Figure 5.18 Number of satisfied constraints: six-bus test with no bad data 
Figure 5.18 shows the convergence characteristic of the MCS estimator. It is clear from that twelve double inequality constraints have been satisfied in this test. Therefore, the convergence characteristics of the MCS may be used as an indication for the number of good and bad measurements in the set.

To simulate an error in telemetry, a single outlier has been introduced, where the correct real power injection at bus1 has been deliberately changed. As in the previous test, the population size $=100$, crossover $=0.8$, mutation $=0.09$, elitism rate $=80 \%$ and tolerance of $\tau= \pm 5 \%$ has been applied to the measurements.

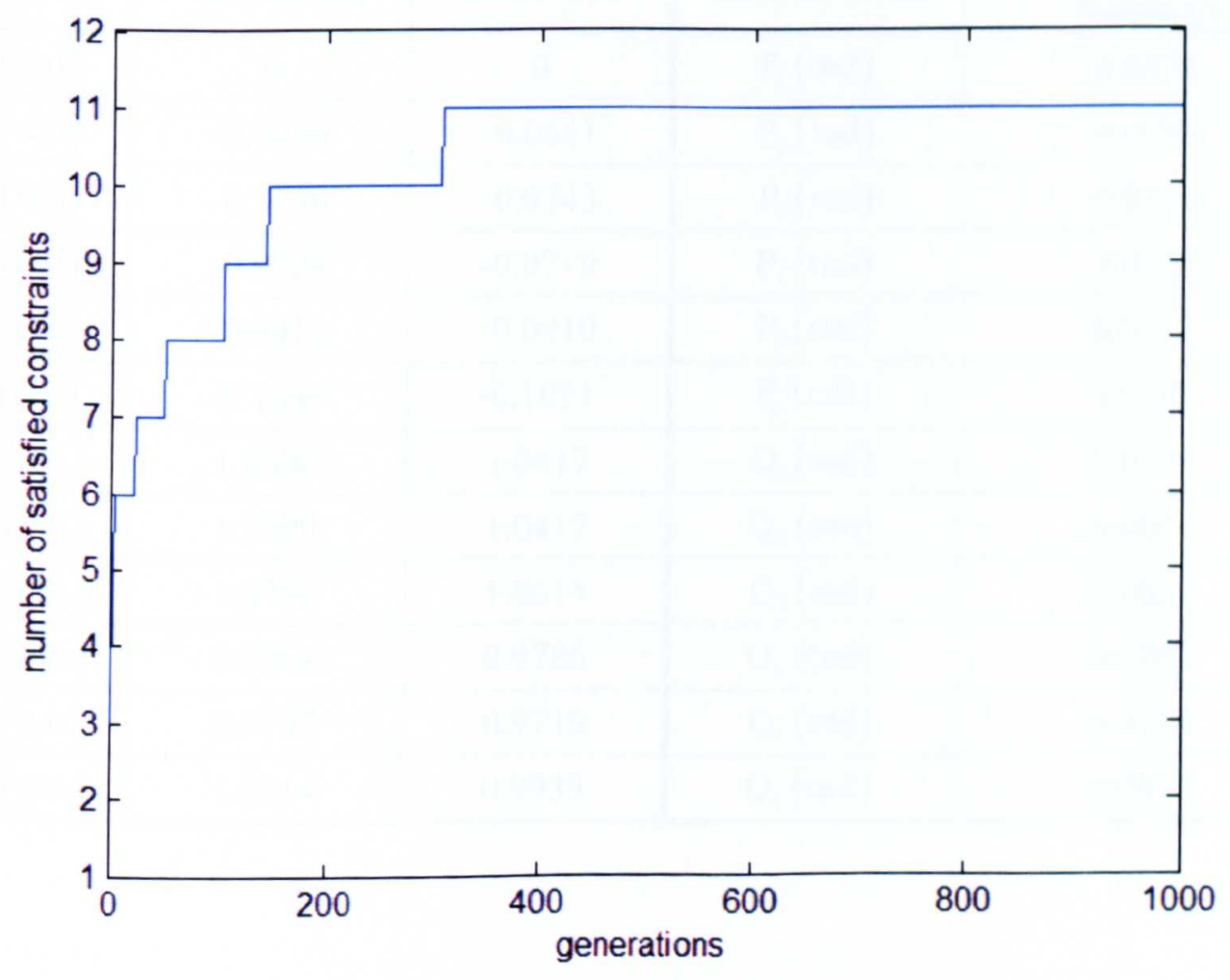

Figure 5.19 Number of satisfied constraints: six-bus test with a single bad data 
As expected, the convergence characteristic in figure 5.19 , shows clearly that a maximum of eleven constraints are satisfied in this test. Table 5.2 shows the true and estimated states of the six-bus system. The non exact fit property of the MCS estimator is the reason of the discrepancies in the true and estimated states. Also included in table 5.2, are the measurement residuals, which have a standard deviation of 0.5907 . It is apparent that the residual of the power injection of bus 1 is the only residual larger than the standard deviation.

Table 5.2 Outcome of MCS estimator with single outlier in the measurement set

\begin{tabular}{|c|c|c|c|c|}
\hline States & True States & MCS-GA & Measurements & $\begin{array}{c}\text { Measurements } \\
\text { Residuals }\end{array}$ \\
\hline$\theta_{1}(\mathrm{rad})$ & 0 & 0 & $\mathrm{P}_{1}(\mathrm{rad})$ & 2.0576 \\
\hline$\theta_{2}(\mathrm{rad})$ & -0.0650 & -0.0641 & $\mathrm{P}_{2}(\mathrm{rad})$ & 0.0099 \\
\hline$\theta_{3}(\mathrm{rad})$ & -0.0756 & -0.0743 & $\mathrm{P}_{3}(\mathrm{rad})$ & 0.0124 \\
\hline$\theta_{4}(\mathrm{rad})$ & -0.0729 & -0.0719 & $\mathrm{P}_{4}(\mathrm{rad})$ & 0.0186 \\
\hline$\theta_{5}(\mathrm{rad})$ & -0.0912 & -0.0910 & $\mathrm{P}_{5}(\mathrm{rad})$ & 0.0022 \\
\hline$\theta_{6}(\mathrm{rad})$ & -0.1042 & -0.1021 & $\mathrm{P}_{6}(\mathrm{rad})$ & 0.0308 \\
\hline$\left|\mathrm{V}_{1}\right|($ p.u. $)$ & 1.0500 & 1.0417 & $\mathrm{Q}_{1}(\mathrm{rad})$ & 0.0174 \\
\hline$\left|\mathrm{V}_{2}\right|$ (p.u.) & 1.0500 & 1.0417 & $\mathrm{Q}_{2}(\mathrm{rad})$ & 0.0041 \\
\hline$\left|\mathrm{V}_{3}\right|$ (p.u.) & 1.0700 & 1.0615 & $\mathrm{Q}_{3}(\mathrm{rad})$ & 0.0031 \\
\hline$\left|\mathrm{V}_{4}\right|$ (p.u.) & 0.9864 & 0.9786 & $\mathrm{Q}_{4}(\mathrm{rad})$ & 0.0082 \\
\hline$\left|\mathrm{V}_{5}\right|$ (p.u.) & 0.9797 & 0.9719 & $\mathrm{Q}_{5}(\mathrm{rad})$ & 0.0164 \\
\hline$\left|\mathrm{V}_{6}\right|$ (p.u.) & 1.0014 & 0.9935 & $\mathrm{Q}_{6}(\mathrm{rad})$ & 0.0050 \\
\hline
\end{tabular}


Table 5.3 Outcome of MCS estimator with two outliers in the measurement set

\begin{tabular}{|c|c|c|c|c|}
\hline States & True States & MCS-GA & Measurements & $\begin{array}{c}\text { Measurements } \\
\text { Residuals }\end{array}$ \\
\hline$\theta_{1}(\mathrm{rad})$ & 0 & 0 & $\mathrm{P}_{1}(\mathrm{rad})$ & 2.0483 \\
\hline$\theta_{2}(\mathrm{rad})$ & -0.0650 & -0.0593 & $\mathrm{P}_{2}(\mathrm{rad})$ & 0.0009 \\
\hline$\theta_{3}(\mathrm{rad})$ & -0.0756 & -0.0695 & $\mathrm{P}_{3}(\mathrm{rad})$ & 0.0202 \\
\hline$\theta_{4}(\mathrm{rad})$ & -0.0729 & -0.0677 & $\mathrm{P}_{4}(\mathrm{rad})$ & 0.0252 \\
\hline$\theta_{5}(\mathrm{rad})$ & -0.0912 & -0.0864 & $\mathrm{P}_{5}(\mathrm{rad})$ & 0.0000 \\
\hline$\theta_{6}(\mathrm{rad})$ & -0.1042 & -0.0974 & $\mathrm{P}_{6}(\mathrm{rad})$ & 0.0314 \\
\hline$\left|\mathrm{V}_{1}\right|($ p.u. $)$ & 1.0500 & 1.0483 & $\mathrm{Q}_{1}(\mathrm{rad})$ & 2.7739 \\
\hline$\left|\mathrm{V}_{2}\right|$ (p.u.) & 1.0500 & 1.0383 & $\mathrm{Q}_{2}(\mathrm{rad})$ & 0.0101 \\
\hline$\left|\mathrm{V}_{3}\right|$ (p.u.) & 1.0700 & 1.0547 & $\mathrm{Q}_{3}(\mathrm{rad})$ & 0.0080 \\
\hline$\left|\mathrm{V}_{4}\right|$ (p.u.) & 0.9864 & 0.9755 & $\mathrm{Q}_{4}(\mathrm{rad})$ & 0.0298 \\
\hline$\left|\mathrm{V}_{5}\right|$ (p.u.) & 0.9797 & 0.9657 & $\mathrm{Q}_{5}(\mathrm{rad})$ & 0.0346 \\
\hline$\left|\mathrm{V}_{6}\right|$ (p.u.) & 1.0014 & 0.9871 & $\mathrm{Q}_{6}(\mathrm{rad})$ & 0.0000 \\
\hline
\end{tabular}

Results of another test with two outliers, real and reactive power injections of bus 1 , are shown in table 5.3 with convergence characteristics illustrated in figure 5.20. As infered from the figure, only ten constraints can be satisfied. The standard deviation of the residuals is 0.9451 . It is apparent that residuals corresponding to real and reactive power injection of busl are larger than the standard deviations. 


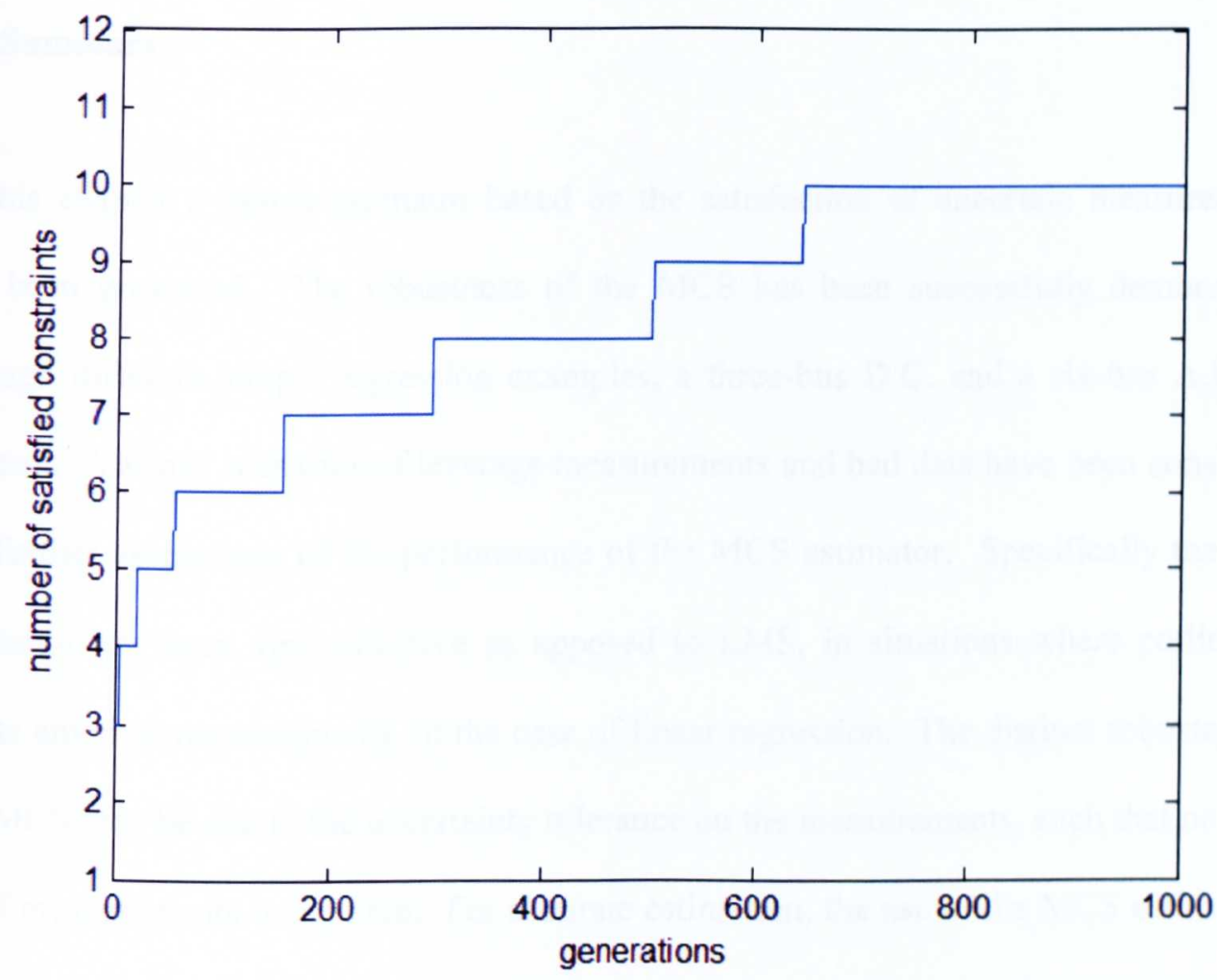

Figure 5.19 Number of satisfied constraints: six-bus test with two bad data

From the convergence characteristics, it appears that the estimation procedure for twelve measurements of the six-bus test system is completed after a quite high number of iterations. It is expected that with the application of the MCS estimator on larger networks with higher numbers of measurements would be quite expensive in terms of execution time. This drawback is mainly due to the difficult search space of the objective function, which can be visualized as a campus map, where different buildings with different heights are scattered all over the area of the campus. The surface of each entity in the campus is normally flat, i.e. there is no gradient to drive the GA. Hence, application of the MCS estimator to the non-linear A.C. state estimation problem may be inefficient and not suitable for practical application, as yet. 


\subsection{Summary}

In this chapter a robust estimator based on the satisfaction of uncertain measurements, has been presented. The robustness of the MCS has been successfully demonstrated through different simple regression examples, a three-bus D.C. and a six-bus A.C. test systems. Various scenarios of leverage measurements and bad data have been considered for further assessment of the performance of the MCS estimator. Specifically the MCS estimator has been very effective as apposed to LMS, in situations where collinearity exists amongst measurements, in the case of linear regression. The distinct robustness of the MCS may be due to the uncertainty tolerance on the measurements, such that no exact fit of measurements is required. For accurate estimation, the use of the MCS estimator is recommended for identification and elimination of the outliers, prior the use of any nonrobust estimator. On the other hand, the high computational time burden of the MCS estimato: was apparent due to the exhaustive nature of the proposed estimator and the lack of drive that the proposed objective function exhibits. Possibly, one way to deal with this drive problem is to use another search method in conjunction with RGA that does not rely on drive that of the objective function. 


\section{CHAPTER SIX: CONCLUSION}

\subsection{Introduction}

As a special case of the state estimation problem, the load-flow problem has been investigated. Although it has been claimed in the literature that GAs can only provide near optimal solution for the load-flow problem, in chapter two of this thesis it has been demonstrated that a real-coded GA is able to provide an exact solution to the problem. The proposed RGA can be regarded as an efficient method when compared to other evolutionary methods for solving the load-flow problem. It is important to mention that the RGA approach is not expected to be practical for on-line applications, but can be useful off-line studies where multiple solutions are suspected and also forms a basis for some more advanced methods presented later in this thesis.

Two formulations of uncertainty analysis in power system state estimation are presented in chapter three. The uncertainty is modelled via deterministic upper and lower bounds on measurement errors, which take into account known meter accuracies. Linear and nonlinear and formulations are provided to estimate the upper and lower bounds on the states. Both methods provided almost identical estimates. It is concluded from execution time analysis that (WLS-LP) is faster than (SQP) and more appropriate for uncertainty interval estimation in larger power networks. Consequently, analysis of uncertainty in power system state estimation with (WLS-LP) is applied on six-bus, IEEE 30-bus and the IEEE 118-bus test systems, for which a conventional WLS estimator is used to obtain 
point estimates of the states, and then a series of LP solutions is used to compute the tightest possible bounds on the states and other quantities of interest. It is deduced that the method offers useful additional information to the power system operator. By examining bounds on the estimates one can infer the quality of the metering configuration and determine the proximity of estimated quantities to voltage and flow limits with greater confidence. An interesting "cross-over" phenomena can occur in cases where network parameter errors exist, and this may be useful for model validation purposes.

The uncertainty analysis in power system state estimation is extended to include the uncertainty of the network parameters. In chapter four, the uncertainty is modelled via deterministic upper and lower bounds on measurement errors, which take into account known meter accuracies. Parametric uncertainties are also known and bounded. A nonlinear formulation is provided to estimate the upper and lower bounds on the states and the parameters. The non-linear problem is solved by Sequential Quadratic Programming (SQP) techniques. Uncertainty analysis in power system state estimation offers useful additional information to the power system operator. By examining bounds on the estimates one can infer the quality of the metering configuration and determine the proximity of estimated quantities to voltage and flow limits with greater confidence.

In chapter five a robust estimator based on the satisfaction of uncertain measurements, has been presented. The robustness of the MCS has been successfully demonstrated through different simple regression examples, a three-bus D.C. and a six-bus A.C. test 
systems. Various scenarios of leverage measurements and bad data have been considered for further assessment of the performance of the MCS estimator. Specifically the MCS estimator has been very effective as apposed to LMS, in situations where collinearity exists amongst measurements, in the case of linear regression. The distinct robustness of the MCS may be due to the uncertainty tolerance on the measurements, such that no exact fit of measurements is required. For accurate estimation, the use of the MCS estimator is recommended for identification and elimination of the outliers, prior the use of any nonrobust estimator.

\subsection{Proposal for future work}

The solution of non-linear load-flow formulation by means of genetic algorithms may be further improved, (in terms of accuracy and convergence speed), by using and adaptive range genetic algorithms. The essence of their idea is to adapt the population toward promising design regions during the optimization process, which enables efficient and robust search in good precision while keeping the string length small. Adaptive Range Genetic Algorithms (ARGAs) are a quite new approach, which employs dynamic coding scheme proposed by Arakawa and Hagiwara [118] for binary-coded GAs to treat continuous design space. In addition, a real-coded ARGA is also introduced by Oyama $e t$ al. in [119], which possesses both advantages of the binary-coded ARGA and the floating-point representation to overcome the problems of having a large search space that requires continuous sampling. For confirmation, a full description and implementation of the proposed method in [118] is shown in appendix A. The result 
confirms that the ARGA consistently finds better solutions than the conventional realcoded genetic algorithms do.

In this research, the uncertainty in power system state is modeled by a probabilistic approach, but such a representation may not always be adapted for representation of meter inaccuracies, since the formulations proposed in this thesis assume transducer tolerances $\underline{\tau}$ must be known and fixed. As mentioned, in realty the instrument inaccuracies will become unknown as the instruments age under the action of various unknown processes and as the instruments are not recalibrated. It must be noted that measurement recalibration is rarely carried out in a systematic manner by utilities [ 55 , 56]. This is mainly due to the fact that large numbers of measurements exist in a power network and the time and expertise required to check each individual transducer would be expensive. For future research, a fuzzy representation, based on the possibility theory, seems to be suitable for modeling of uncertainty may be used for uncertainty modeling of power system state estimation. A truncated triangular probability-possibility transformation is introduced in [120] where a unimodal and symmetric probability distribution may be used for computation of the bounds, (i.e. $\underline{\underline{z}}^{\prime}, \underline{z}^{\prime \prime}, \Delta \underline{z}^{\prime}, \Delta \underline{z}^{\prime \prime}$ of equations $3-7,3-8,3-11$ and 3-12 ), of the double inequality constraints formulations presented in this thesis. Moreover, another fuzzy approach Mauris et al. [121] may be used for the same objective which consists of representing the uncertainty in the measurements by a family of intervals of confidence stacked atop one another, which define the upper bound of the probability distributions consistent with these intervals of 
confidence. This approach is compatible with the ISO Guide for the expression of uncertainty in measurement, and is particularly interesting because it allows both the handling of specificity and uncertainty of measurement. 


\section{Appendix A}

\section{Adaptive Real Range Genetic Algorithms (ARGAs)}

Adaptive Range Genetic Algorithms (ARGAs) are a new approach, which employ dynamic coding schemes proposed by Arakawa and Hagiwara [118] for binary-coded GAs to treat a continuous design space. The essence of their idea is to adapt the population toward promising design regions during the optimization process, which enables efficient and robust search in good precision while keeping the string length small. Furthermore, ARGAs eliminate prior definition of boundaries of the search regions since ARGAs distribute design candidates according to the normal distributions of the design variables in the present population.

In conventional binary-coded GAs, discrete values of real design variables are given by evenly discretizing prior-defined search regions for each design variable according to the length of the binary substring. Traditionally, a conversion from binary to real number of a design variable $x$, for the searching range $\left[x_{t, \min }, x_{1, \max }\right]$ may be accomplished by

$$
R\left(p_{i}\right)=x_{i, \text { min }}+\frac{C\left(p_{i}\right) \cdot\left(x_{i, \text { max }}-x_{i, \text { min }}\right)}{2^{m}-1}
$$

where $p_{i}$ denote a binary chromosomes, $C\left(p_{i}\right)$ denotes an integer after conversion of

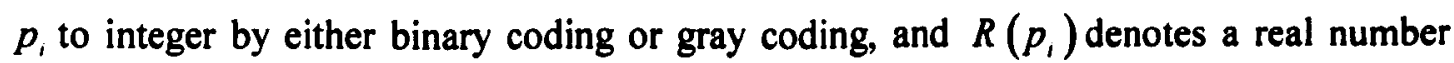


after conversion to real number. One disadvantage of this conversion method is that it is almost impossible to obtain an exact solution due to the fixed discretization procedure of the searching range.

In binary-coded ARGAs, when vectors of design variables are given as individuals, a specific number of bits are assigned it each design variable. For instance, when there are three variables, and 2, 3, 4 bits are assigned for each variable; chromosomes will be composed of 9 bits. For each variable, we will consider the following processes. As the most popular way of expressing continuous numbers the conventional method, equation (A.1), evenly divides a given search range or boundary. However, in the proposed method, we can calculate mean $\left(\mu_{i}\right)$ and standard deviation $\left(\sigma_{i}\right)$ for each design variable in each generation. By using these values, we can determine some sort of distribution such as a normal distribution normalized to the maximum value of 1 as

$$
N(x)=\exp \left(-(x-\mu)^{2} / 2 / \sigma^{2}\right)
$$

This distribution shows the situation of each generation and it adapts automatically to the best-fit searching range in some generation. Using this distribution, we can divide the vertical axis evenly to give continuous variables as

$$
R\left(p_{i}\right)=\left\{\begin{array}{l}
\mu_{i}-\sqrt{-2 \sigma_{i}^{2} \ln \left(L B_{i}+\frac{\left(U B_{i}-L B_{i}\right) C\left(p_{i}\right)}{2^{m-1}-1}\right)}, \text { for } C\left(p_{i}\right) \prec 2^{m-1} \\
\mu_{i}+\sqrt{-2 \sigma_{i}^{2} \ln \left(U B_{i}-\frac{\left(U B_{i}-L B_{i}\right)\left(C\left(p_{i}\right)-2^{m-1}\right)}{2^{m-1}-1}\right)}, \text { for } C\left(p_{i}\right) \succ 2^{m-1}
\end{array}\right.
$$


where $U B_{i}$ and $L B_{i}$ are system parameters shown in figure A.l $\mu_{i}$ is the mean value of the population remaining in the preceding generation, and $\sigma_{i}$ is the standard deviation. $\sigma_{i}$ is concerned with the determination of searching range, and we can treat upper and lower bounds for $\sigma_{i}$ as system parameters $\left(\sigma_{i, \min }, \sigma_{i, \max }\right) . \sigma_{i, \max }$ aims to prevent extension of the searching range in the first few generation, and $\sigma_{i, \min }$ aims to prevent the searching range becoming too narrow to keep an efficient range. The vertical axis of figure A.1 is given to determine the discrete values $\left(\begin{array}{lll}x_{1} & \text { to } & x_{7}\end{array}\right)$; thus it has no physical meaning. 'I' means that we divide the vertical axis evenly, and $x_{i}$ in the horizontal axis represents the discrete values given by the proposed decoding method. For example, if we obtained the integer 3 after initial decoding, the real number will be $x_{3}$. As for the initial generation, we use a conventional method because we do not have any information on mean values or standard deviations of the preceding generation.

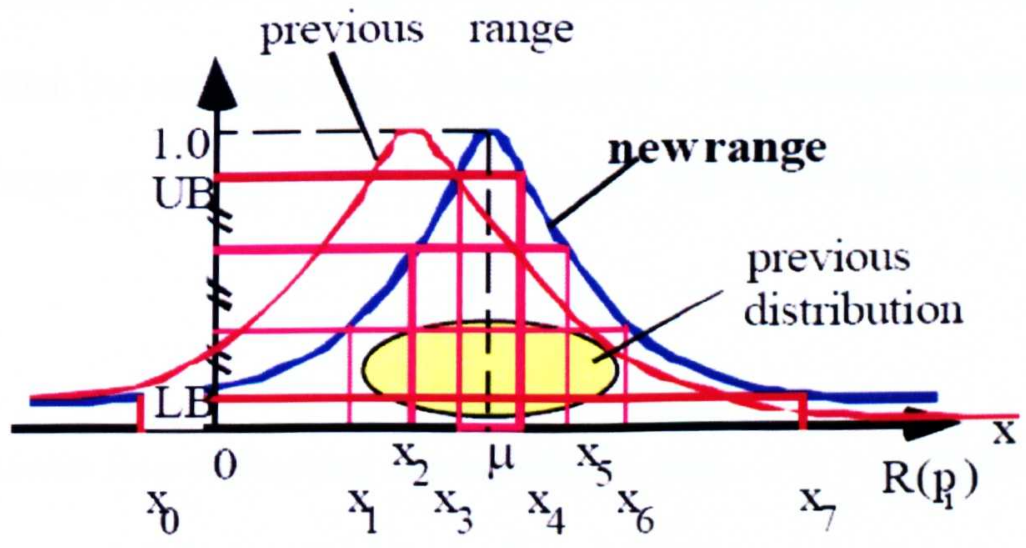

Figure A.1 Adaptive range GAs 
In the proposed method, the searching range varies according to the distribution of population in the preceding generation as shown in figure A.1. When distribution tends to concentrate at the upper or lower side of the mean value, the searching range is shifted to that direction, as shown in figure A.1. However, these tendencies will not liquidate in single generation even when it becomes unsuitable. Thus, the searching range sometimes goes beyond the suitable range to obtain the global optimum. In such a case, it is possible to miss the global optimum especially in a multi-peak problem. Even if the searching range can be returned, to the suitable range, one of the easiest ways of solving these problems is to change the searching range in every other specific generation, which is sufficient for liquidating these tendencies. However, it might be difficult to predict the number of generations needed to liquidate tendencies, and this will also slow down the adaptability of the searching range. Thus it is not the appropriate solution. Another way it to narrow down adaptability to adjust the searching range to the suitable range, especially when the optimum solution will be beyond the initial given range. In order to adjust the searching range to the range suitable for obtaining the global optimum rapidly, we need to widen the searching range; for that purpose, it is preferable to assign a smaller $L B_{i}$, and a larger $\sigma_{i, \min }$ value. This leads to conflicting requirements in setting system parameters.

One of the reasons for overshooting and missing the range near the global optimum lies in the phenomenon that the best solution to all preceding generations becomes the outside the searching range. In order to keep it within the searching range, we treat standard 
deviation, determines the searching range separately for both upper and lower sides, as shown in the following equations

$$
\begin{aligned}
& \text { for Max } \text { value }_{i} \succ \mu_{i}
\end{aligned}
$$

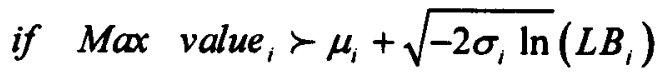

$$
\begin{aligned}
& \text { then } \sigma_{i, \text { new }}^{R}=\frac{\text { Max } \text { value }_{i}-\mu_{i}}{\sqrt{-2 \ln \left(L B_{i}\right)}} \\
& \text { for Max value, } \prec \mu_{i} \\
& \text { if Max } \text { value }_{i} \prec \mu_{i}-\sqrt{-2 \sigma_{1} \ln }\left(L B_{i}\right) \\
& \text { then } \sigma_{i, \text { new }}^{L}=\frac{-M a x \text { value }_{i}+\mu_{i}}{\sqrt{-2 \ln \left(L B_{i}\right)}}
\end{aligned}
$$

Max value, means the design variable $i$ that gives the best-fit function value from the initial generation to the preceding generation to the preceding generation, $\sigma_{i \text {,new }}^{R}, \sigma_{i, \text { new }}^{L}$ denote the new system parameters for right and left hand sides. Using this new operation, the searching range will be changed from the "old range" which includes Max value, .

The following are characteristics of the proposed method

- The real searching range will change with every generation according to the remaining individuals' mean values and standard deviations. Its adapt the condition for the optimization process. Thus, even if the optimum solution is 
outside the range of the initially given searching range, we can obtain the optimum solutions.

- As the generation goes by, mean values will become some unique values and standard deviation will decrease. This means that we do not need to assign many bits in order to improve the accuracy of the solution (Save computational memory and improve convergence).

\section{Illustrative examples}

In order to show effectiveness, the proposed method has been applied and verified on the following simple function:

$$
f=100000-\left(x_{1}-150\right)^{2}-\left(x_{2}-5.5\right)^{2}-\left(x_{3}+150\right)^{2}-\left(x_{4}+5.5\right)^{2}
$$

The objective is to maximize $f$. For every design variable, an initial searching range of lower boundary $=0.0$ and upper boundary $=10.0$ is assigned. 3 bits is assigned for all designed variables. Each generation has a population size $=50 \%$, crossover $=80 \%$, and mutation rate $=0.01 . L B=0.9$ and $U B=0.1$ for convenience, the standard deviations $\left(\sigma_{i, \min }, \sigma_{i, \max }\right)$ has no assignment and limitation. The convergence characteristics of the fitness function and the mean value of each designed variables are shown in figure A.2 and A3. For this particular problem, a quite good convergence can be seen from figure A.2. It can be seen from figure A.3 we can obtain the results that are initially not within the given searching range. Therefore, it is confirmed that true optimum solution can be found without having any pre-knowledge searching range. 


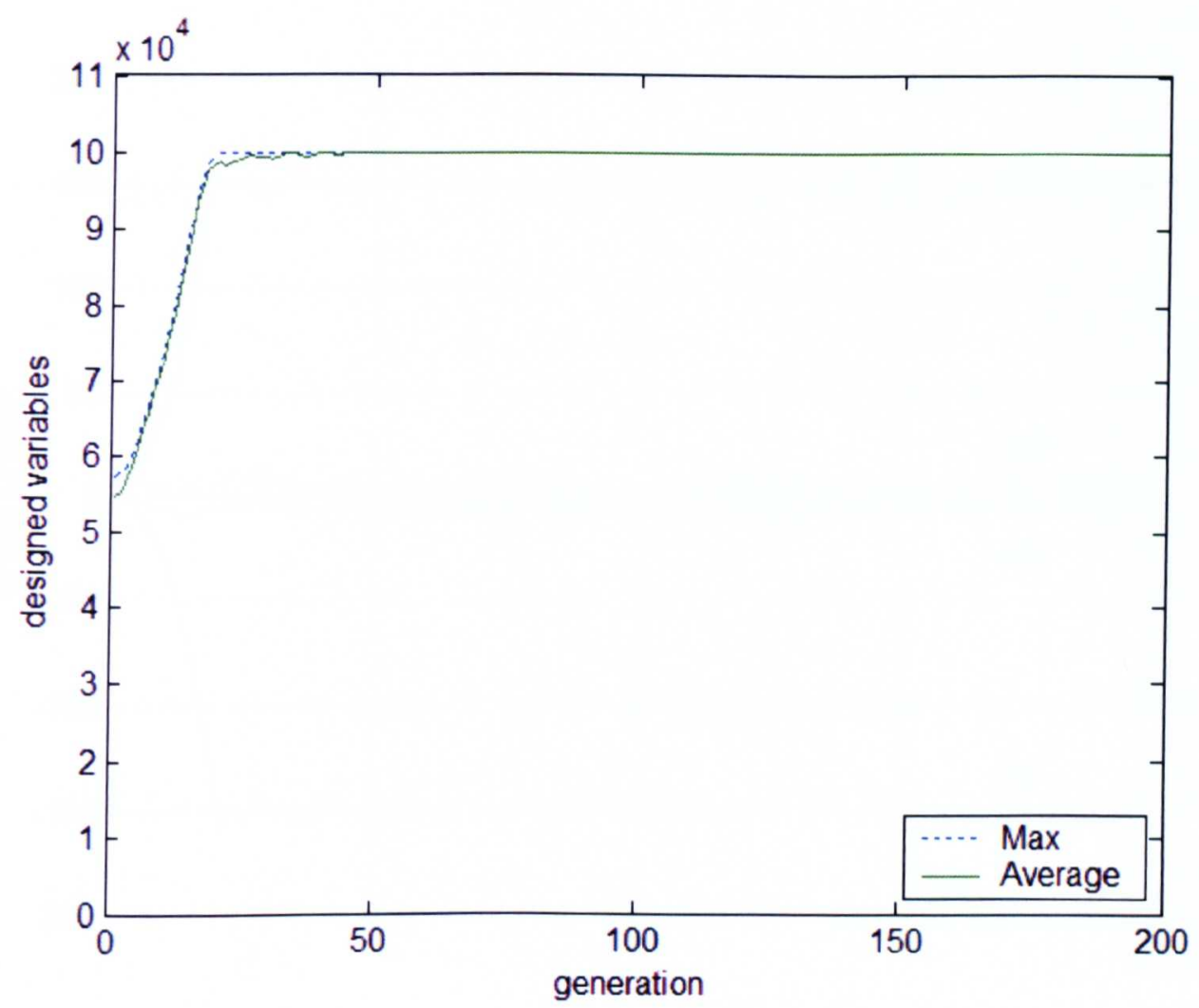

Figure A.2 Fitness function 


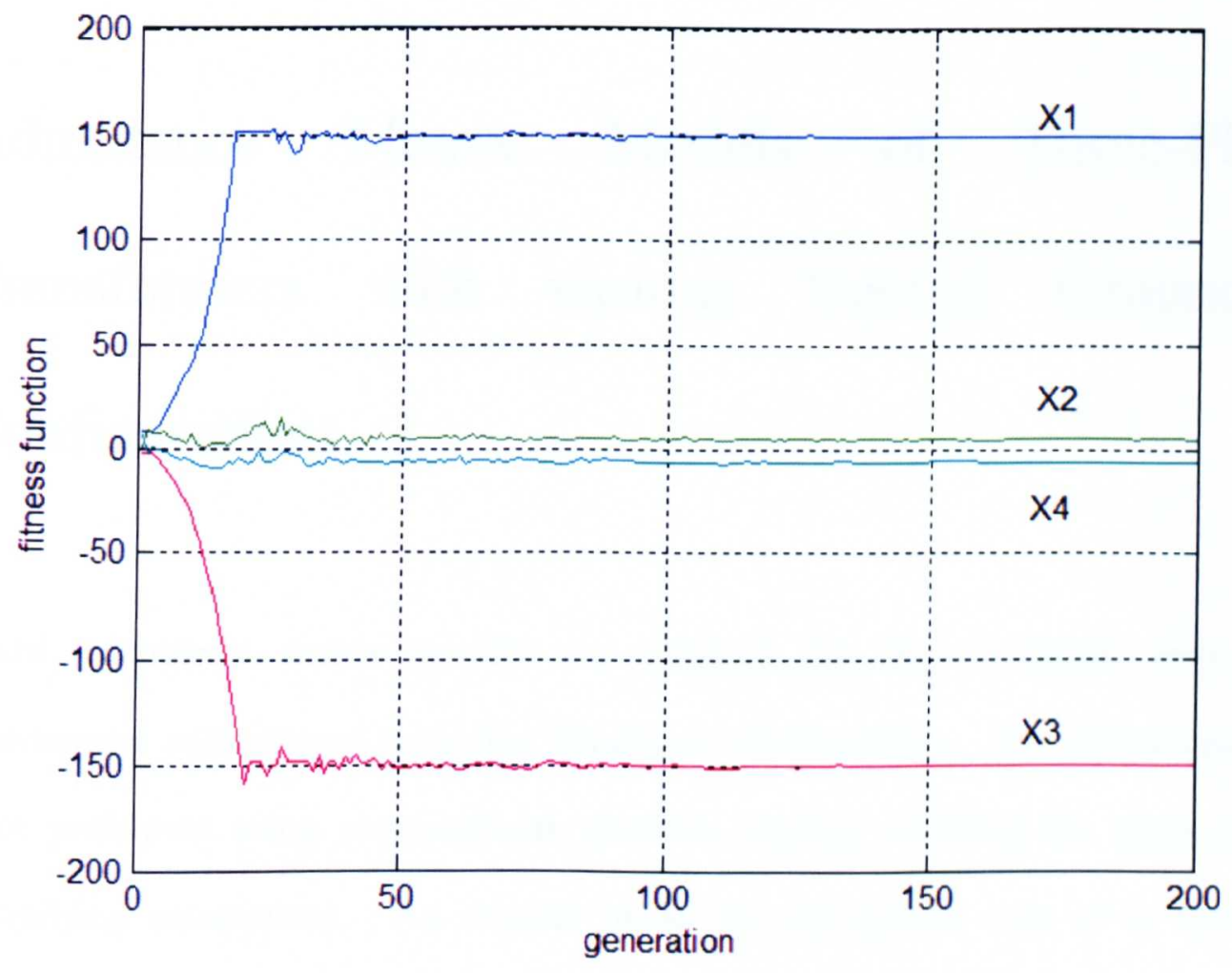

Figure A.3 Designed variables 


\section{Appendix B}

\section{Admittance Matrix Models of Three-Phase Transformers with various Neutral Grounding \\ Configurations}

Nodal admittance matrix models are obtained for Wye-connected three-phase transformers with various grounding impedance configurations. The derivations have been performed using computational symbolic algebra, avoiding the necessity for simplifying assumptions. An original model for the general case of a Wye-Wye transformer, with grounding impedances on both primary and secondary neutrals, is given. It is shown that this model does not correspond to the simple combination of the previously known formulae for cases where neutral impedance occurs on either the primary side only or the secondary side only. It is also demonstrated that the new model simplifies to the well-known limiting cases for solid grounding and no grounding. A model is also given for multiple transformers sharing a single neutral grounding impedance. The models obtained here may form a useful basis for further research in applying the uncertainty estimation techniques presented in this thesis to unbalanced power-flow models. 
The increasing need to study unbalanced conditions in transmission and distribution networks has led to the development of three-phase load-flow and short-circuit study programs. The use of phase co-ordinates, rather than sequence components, has become more popular recently $[122,123]$. This may be because the sequence components are only independent of each other when the mutual inductance between pairs of phases are all equal (i.e. the phase conductors, or windings, have geometrical symmetry). It is common practice to apply a nodal admittance matrix formulation for both loadflow and short-circuit programs, and it is then necessary to obtain admittance matrix terms that accurately represent the possible three-phase transformer configurations encountered in power networks. Suitable models are available in the literature for common, and some of the less common, configurations.

This appendix develops the admittance matrix model for the general case of a Wye-Wye transformer with neutral grounding impedances on both the primary and secondary sides. It is shown that this general model is consistent with the well-known cases for solid grounding and/or no grounding on either side. The general model is also consistent with the previously given case of impedance grounding on either the primary side or the secondary side (but not both). Interestingly, it is found that simply adding the appropriate terms for grounding impedance on the primary side, together with similar terms arising from grounding impedance on the secondary side, does not produce the correct result when both are present. Furthermore, it is noted that the correct model for solid grounding on one side, with no grounding on the other side (often referred to as WyeG -Wye, or 
Wye-WyeG), is correctly given in the earlier reference works but has sometimes been misprinted in recent publications.

In practice, two or more transformers may share a single grounding impedance. The general model for such cases is also given. Derivation of the various models presented here has been greatly facilitated by the use of computerised algebra (e.g. the MathCAD package). This approach allows various configurations to be analysed rapidly and also allows any of the usual simplifying assumptions to be relaxed where necessary. For example, it is straightforward to include different values for self-admittance and mutual admittance, and to allow for asymmetry in the mutual admittances arising from the transformer geometry (as for 3- or 5-limb Iron cores). However, for the sake of brevity and clarity, this paper will discuss the simplified cases, which can be readily compared with previously known results.

Wye-Wye Transformer with Neutral Grounding Impedances: To consider the most general case for a Wye-Wye transformer, figure B.1 shows neutral grounding via impedances on both the primary and secondary sides. 


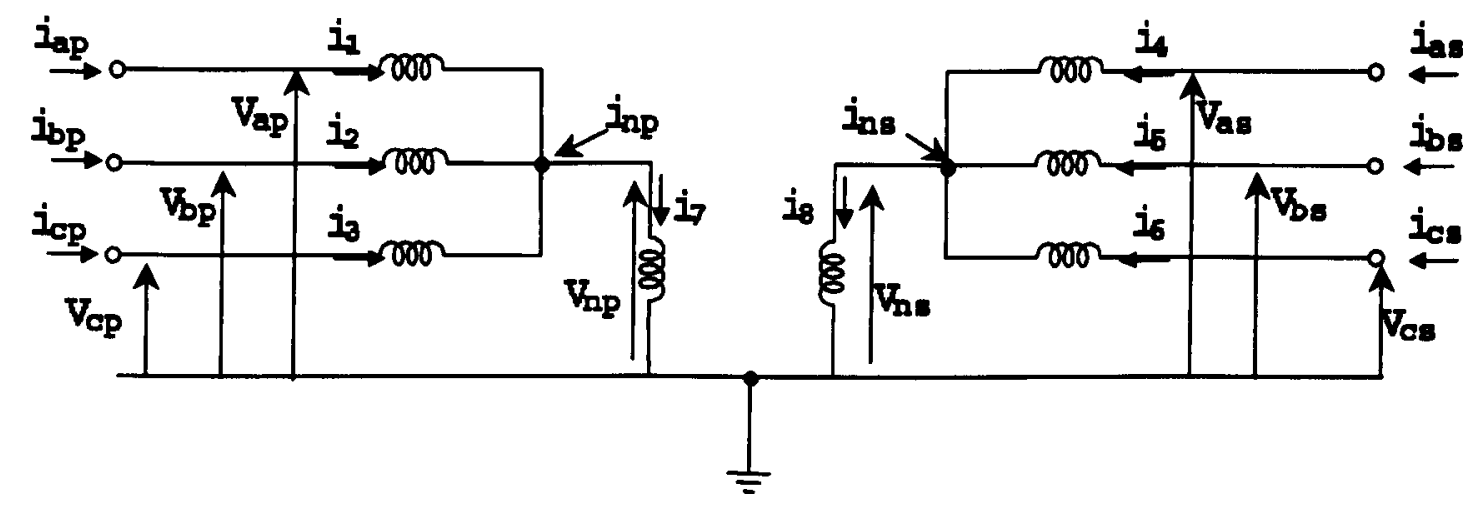

Figure B.1 Wye-Wye transformer with neutral impedance on primary and secondary side

The primitive network for this case is shown in figure B.2.

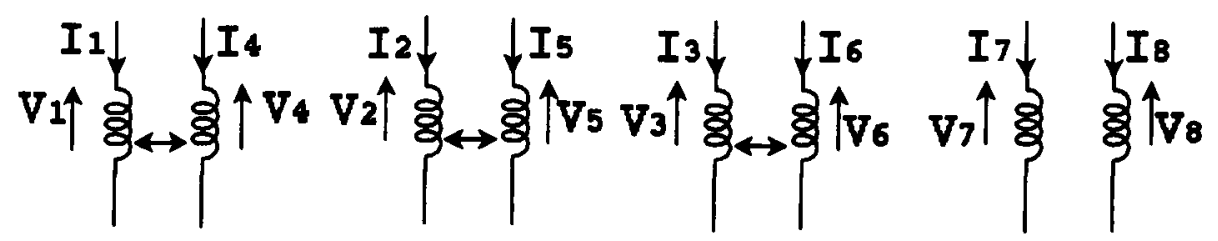

Figure B.2 corresponding primitive network

The nodal admittance matrix for this case can be obtained as follows, using the primitive admittance matrix and the appropriate connection matrix [123]. It is assumed that all mutual admittances $(m)$ are equal and that the leakage admittance $(y)$ is constant throughout.

$$
Y_{\text {node }}=C^{T} \cdot Y_{\text {prim }} \cdot C
$$

where 


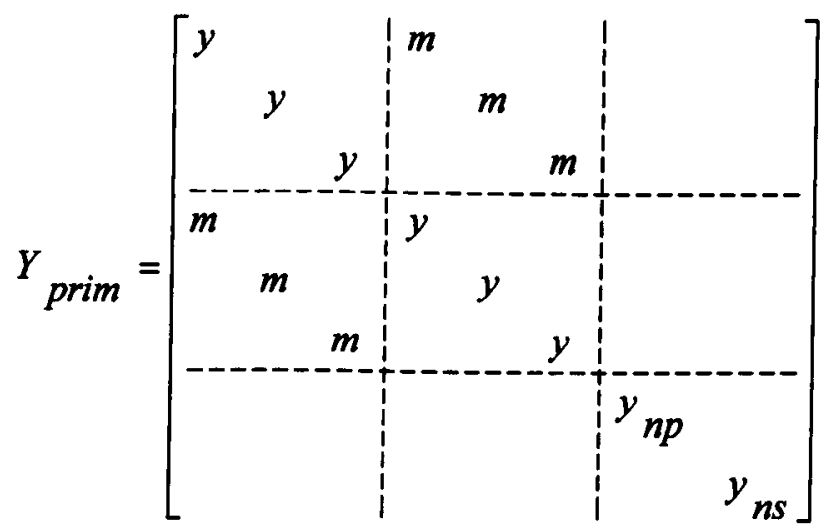

and

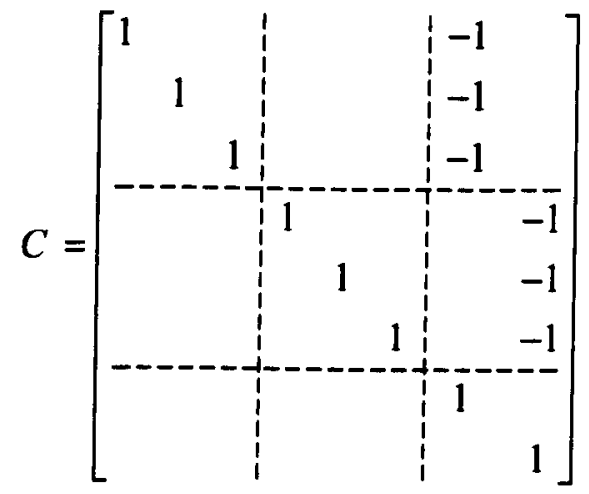

\{zero entries are omitted for clarity\}

This results in the following $(8$ by 8$)$ admittance matrix:

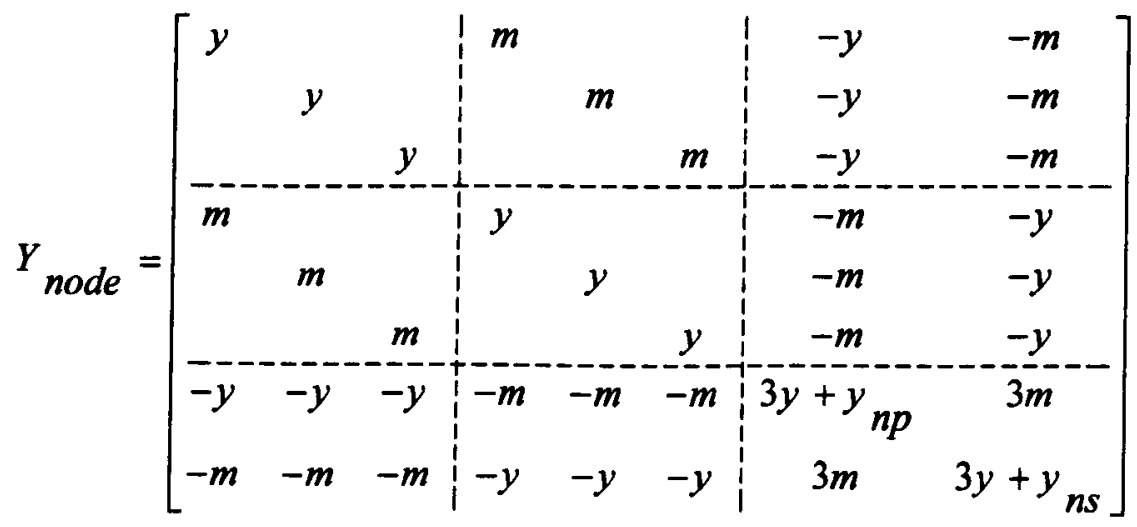


The above matrix should be reduced to a 6 by 6 nodal admittance matrix by eliminating the neutral point nodes (noting that the injected currents at the neutral points, $i_{n p}$ and $i_{n s}$, are zero), considering the ( 8 by 8 ) admittance matrix in partitioned form.

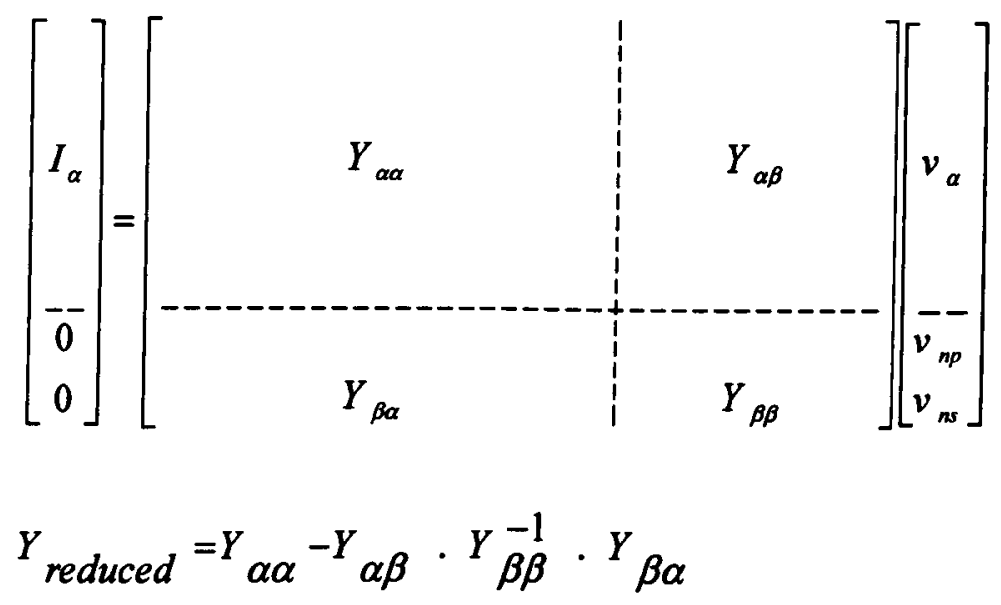

Using computerised symbolic algebra, it is very straightforward to obtain expressions for each admittance matrix element. Indeed, the assumptions of equal mutual admittances between primary and secondary windings on each phase, and zero mutual admittance between different phases, implicit in figure B.2, can easily be relaxed if appropriate data is available. However, these assumptions, together with the further assumption that $y=$ $m$, will be made here for simplicity and brevity and so that the results obtained can be compared with earlier results. 
The admittance matrix is then:

$$
Y_{\text {reduced }}=\left[\begin{array}{c:c}
Y & Y \\
Y & Y
\end{array}\right]
$$

where

$$
Y=\left[\begin{array}{ccc}
h & -g & -g \\
-g & h & -g \\
-g & -g & h
\end{array}\right]
$$

and

$$
\begin{aligned}
& h=\frac{y\left(2 y y_{n p}+2 y y_{n s}+y_{n p} y_{n s}\right)}{3 y y_{n p}+3 y y_{n s}+y_{n p} y_{n s}} \\
& g=\frac{y^{2}\left(y_{n p}+y_{n s}\right)}{3 y y_{n p}+3 y y_{n s}+y_{n p} y_{n s}}
\end{aligned}
$$

This general case can be compared to well-known results in the literature. For example, if we take the limit as $y_{n p}$ tends to infinity and $y_{n s}$ tends to zero (solid grounding on primary and no grounding on secondary) we obtain:

$$
h=\frac{2}{3} y \quad \text { and } \quad g=\frac{y}{3}
$$

This corresponds to the correct result given in reference [122]. However, it should be noted that some recent references have misprinted this case. The other limiting cases of 
solid grounding and no grounding give results that correspond to those available in the literature.

If we take the limit as $y_{n s}$ tends to infinity but $y_{n p}$ retains a finite value, we have impedance grounding on one side of the transformer. This case is not widely quoted in the literature, but it is analysed in reference [124] and also stated in reference [125]. In this case:

$$
Y=\left[\begin{array}{ccc}
y-c & -c & -c \\
-c & y-c & -c \\
-c & -c & y-c
\end{array}\right]
$$

where $c=\frac{y^{2}}{3 y+y_{n p}}$

Given the above formulae for impedance grounding on one side, it might be assumed that similar correction terms $(c)$ can simply be added to cover the case of impedances on both primary and secondary sides, giving a formula such as:

$$
c=\frac{y^{2}}{3 y+y_{n p}}+\frac{y^{2}}{3 y+y_{n s}}
$$

Comparison of the above with Equation 3 shows that the above formula is not correct however, and that Equation 3 should be implemented in three-phase load-flow and short- 
circuit programs as the general case. Introduction of typical numerical values indicates that the two formulae in question are by no means close approximations.

Wye connected windings with shared neutral grounding impedance:

It is quite common practice to have two or more transformers sharing a single grounding impedance. This configuration has been analysed using the above principles, giving the following admittance sub-matrices for the case where the secondary windings of two transformers share a common grounding impedance:

$$
Y_{\text {reduced }}=\left[\begin{array}{c:c}
Y & Y^{\prime} \\
\hdashline Y^{\prime} & Y
\end{array}\right]
$$

Where

$$
\begin{aligned}
& Y=\left[\begin{array}{ccc}
y-c & -c & -c \\
-c & y-c & -c \\
-c & -c & y-c
\end{array}\right] \text { and } Y^{\prime}=\left[\begin{array}{ccc}
-c & -c & -c \\
-c & -c & -c \\
-c & -c & -c
\end{array}\right] \\
& c=\frac{y^{2}}{6 y+y_{n s}}
\end{aligned}
$$

For the case where the secondaries of three transformers share a common grounding impedance, we obtain. 


$$
\begin{aligned}
& Y_{\text {reduced }}=\left[\begin{array}{ccc}
Y & Y^{\prime} & Y^{\prime} \\
Y^{\prime} & Y & Y^{\prime} \\
Y^{\prime} & Y^{\prime} & Y
\end{array}\right] \\
& \text { with } c=\frac{y^{2}}{9 y+y_{n s}}
\end{aligned}
$$

In the general case of $k$ transformer secondaries sharing a common grounding impedance, we have:

$$
c=\frac{y^{2}}{3 k y+y_{n s}}
$$

Conclusions: Some general cases of three-phase transformer neutral grounding have been analysed. The use of computational symbolic algebra is recommended, as this has greatly facilitated the analysis and will allow various simplifying assumptions to be relaxed where necessary. The admittance matrix model of a Wye-Wye transformer with impedance grounding on both sides has been presented for the first time. The limiting cases of this model are consistent with the well-known models in the literature. It is found that the previously known model for impedance grounding on one side only, does not trivially generalise to the case where both the primary and the secondary are grounded via impedances. A general model is also given for configurations in which two or more transformers share a common grounding impedance. 


\section{Network Data for Test Cases Used in Simulation}

The network data for the six-bus is provided below:

Table C.1 Operating Conditions for six-bus network system

\begin{tabular}{c|c|c|c}
\hline $\begin{array}{c}\text { Bus } \\
\text { number }\end{array}$ & $\begin{array}{c}\text { Voltage } \\
\text { magnitude (PU) }\end{array}$ & $\begin{array}{c}\text { Generation } \\
(\mathrm{MW})\end{array}$ & $\begin{array}{c}\text { Load } \\
\text { (MVAR) }\end{array}$ \\
\hline 1 & 1.0500 & 100 & - \\
\hline 2 & 1.0500 & 50 & - \\
\hline 3 & 1.0700 & 60 & - \\
\hline 4 & 1.0000 & 70 & 70 \\
\hline 5 & 1.0000 & 70 & 70 \\
\hline 6 & 1.0000 & 70 & 70 \\
\hline
\end{tabular}

Table C.2 Impedance and Line Charging Data six-bus network system

\begin{tabular}{c|c|c|c|c}
\hline \multicolumn{2}{c|}{ Line Designation } & Resistance & $\begin{array}{c}\text { Reactance } \\
\text { (PU) }\end{array}$ & $\begin{array}{c}\text { Line Charging } \\
\text { (PU) }\end{array}$ \\
\hline From & To & (PU) \\
\hline 1 & 2 & 0.1000 & 0.2000 & 0.0200 \\
\hline 1 & 4 & 0.0500 & 0.2000 & 0.0200 \\
\hline 1 & 5 & 0.0800 & 0.3000 & 0.0300 \\
\hline 2 & 3 & 0.0500 & 0.2500 & 0.0300 \\
\hline 2 & 4 & 0.0500 & 0.1000 & 0.0100 \\
\hline 2 & 5 & 0.1000 & 0.3000 & 0.0200 \\
\hline 2 & 6 & 0.0700 & 0.2000 & 0.0250 \\
\hline 3 & 5 & 0.1200 & 0.2600 & 0.0250 \\
\hline 3 & 6 & 0.0200 & 0.1000 & 0.0100 \\
\hline 4 & 5 & 0.2000 & 0.4000 & 0.0400 \\
\hline 5 & 6 & 0.1000 & 0.3000 & 0.0300 \\
\hline
\end{tabular}


The network data for the IEEE 14-bus is provided below:

Table C.3 Impedance and Line Charging Data of IEEE 14-bus system

\begin{tabular}{|c|c|c|c|c|}
\hline \multicolumn{2}{|c|}{ Line Designation } & \multirow{2}{*}{$\begin{array}{c}\text { Resistance } \\
\text { (PU) }\end{array}$} & \multirow{2}{*}{$\begin{array}{c}\text { Reactance } \\
(\mathrm{PU})\end{array}$} & \multirow{2}{*}{$\begin{array}{c}\text { Line Charging } \\
(\mathrm{PU})\end{array}$} \\
\hline From & To & & & \\
\hline 1 & 2 & 0.0194 & 0.0592 & 0.0528 \\
\hline 1 & 5 & 0.0540 & 0.2230 & 0.0492 \\
\hline 2 & 3 & 0.0470 & 0.1980 & 0.0438 \\
\hline 2 & 4 & 0.0581 & 0.1763 & 0.0374 \\
\hline 2 & 5 & 0.0570 & 0.1739 & 0.0340 \\
\hline 3 & 4 & 0.0670 & 0.1710 & 0.0346 \\
\hline 4 & 5 & 0.0134 & 0.0421 & 0.0128 \\
\hline 4 & 7 & 0 & 0.2091 & 0 \\
\hline 4 & 9 & 0 & 0.5562 & 0 \\
\hline 5 & 6 & 0 & 0.2520 & 0 \\
\hline 6 & 11 & 0.0950 & 0.1989 & 0 \\
\hline 6 & 12 & 0.1229 & 0.2558 & 0 \\
\hline 6 & 13 & 0.0662 & 0.1303 & 0 \\
\hline 7 & 8 & 0 & 0.1762 & 0 \\
\hline 7 & 9 & 0 & 0.1100 & 0 \\
\hline 9 & 10 & 0.0318 & 0.0845 & 0 \\
\hline 9 & 14 & 0.1271 & 0.2704 & 0 \\
\hline 10 & 11 & 0.0820 & 0.1921 & 0 \\
\hline 12 & 13 & 0.2209 & 0.1999 & 0 \\
\hline 13 & 14 & 0.1709 & 0.3480 & 0 \\
\hline
\end{tabular}

The network data for the IEEE 30-bus is provided below:

Table C.4 Impedance and Line Charging Data of IEEE 30-bus system

\begin{tabular}{|c|c|c|c|c|}
\hline \multicolumn{2}{|c|}{ Line Designation } & \multirow{2}{*}{$\begin{array}{c}\text { Resistance } \\
\text { (PU) }\end{array}$} & \multirow{2}{*}{$\begin{array}{c}\text { Reactance } \\
(\mathrm{PU})\end{array}$} & \multirow{2}{*}{$\begin{array}{c}\text { Line Charging } \\
\text { (PU) }\end{array}$} \\
\hline From & To & & & \\
\hline 1 & 2 & 0.0192 & 0.0575 & 0.0528 \\
\hline 1 & 3 & 0.0452 & 0.1652 & 0.0408 \\
\hline 2 & 4 & 0.0570 & 0.1737 & 0.0368 \\
\hline 3 & 4 & 0.0132 & 0.0379 & 0.0084 \\
\hline 2 & 5 & 0.0472 & 0.1983 & 0.0418 \\
\hline 2 & 6 & 0.0581 & 0.1763 & 0.0374 \\
\hline 4 & 6 & 0.0119 & 0.0414 & 0.0090 \\
\hline 5 & 7 & 0.0460 & 0.1160 & 0.0204 \\
\hline
\end{tabular}


Table C.4 Impedance and Line Charging Data of IEEE 30-bus system (continue)

\begin{tabular}{c|c|c|c|c}
\hline 6 & 7 & 0.0267 & 0.0820 & 0.0170 \\
\hline 6 & 8 & 0.0120 & 0.0420 & 0.0090 \\
\hline 6 & 9 & 0 & 0.2080 & 0 \\
\hline 6 & 10 & 0 & 0.5560 & 0 \\
\hline 9 & 11 & 0 & 0.2080 & 0 \\
\hline 9 & 10 & 0 & 0.1100 & 0 \\
\hline 4 & 12 & 0 & 0.2560 & 0 \\
\hline 12 & 13 & 0 & 0.1400 & 0 \\
\hline 12 & 14 & 0.1231 & 0.2559 & 0 \\
\hline 12 & 15 & 0.0662 & 0.1304 & 0 \\
\hline 12 & 16 & 0.0945 & 0.1987 & 0 \\
\hline 14 & 15 & 0.2210 & 0.1997 & 0 \\
\hline 16 & 17 & 0.0524 & 0.1923 & 0 \\
\hline 15 & 18 & 0.1073 & 0.2185 & 0 \\
\hline 18 & 19 & 0.0639 & 0.1292 & 0 \\
\hline 19 & 20 & 0.0340 & 0.0680 & 0 \\
\hline 10 & 20 & 0.0936 & 0.2090 & 0 \\
\hline 10 & 17 & 0.0324 & 0.0845 & 0 \\
\hline 10 & 21 & 0.0348 & 0.0749 & 0 \\
\hline 10 & 22 & 0.0727 & 0.1499 & 0 \\
\hline 21 & 22 & 0.0116 & 0.0236 & 0 \\
\hline 15 & 23 & 0.1000 & 0.2020 & 0 \\
\hline 22 & 24 & 0.1150 & 0.1790 & 0 \\
\hline 23 & 24 & 0.1320 & 0.2700 & 0 \\
\hline 24 & 25 & 0.1885 & 0.3292 & 0 \\
\hline 25 & 26 & 0.2544 & 0.3800 & 0 \\
\hline 25 & 27 & 0.1093 & 0.2087 & 0 \\
\hline 28 & 27 & 0 & 0.3960 & 0 \\
\hline 27 & 29 & 0.2198 & 0.4153 & 0 \\
\hline 27 & 30 & 0.3202 & 0.6027 & 0 \\
\hline 29 & 30 & 0.2399 & 0.4533 & 0 \\
\hline 8 & 28 & 0.0636 & 0.2000 & 0.0428 \\
\hline 6 & 28 & 0.0169 & 0.0599 & 0.0130 \\
\hline & & & & \\
\hline
\end{tabular}


The network data for the IEEE 118-bus is provided below:

Table C.5 Impedance and Line Charging Data of IEEE 118-bus system

\begin{tabular}{|c|c|c|c|c|}
\hline \multicolumn{2}{|c|}{ Line Designation } & \multirow{2}{*}{$\begin{array}{l}\text { Resistance } \\
\text { (PU) }\end{array}$} & \multirow{2}{*}{$\begin{array}{c}\text { Reactance } \\
\text { (PU) }\end{array}$} & \multirow{2}{*}{$\begin{array}{c}\text { Line Charging } \\
\text { (PU) }\end{array}$} \\
\hline From & To & & & \\
\hline 1 & 2 & 0.0303 & 0.0999 & 0.0254 \\
\hline 1 & 3 & 0.0129 & 0.0424 & 0.0108 \\
\hline 4 & 5 & 0.0018 & 0.0080 & 0.0021 \\
\hline 3 & 5 & 0.0241 & 0.1080 & 0.0284 \\
\hline 5 & 6 & 0.0119 & 0.0540 & 0.0143 \\
\hline 6 & 7 & 0.0046 & 0.0208 & 0.0055 \\
\hline 8 & 9 & 0.0024 & 0.0305 & 1.1620 \\
\hline 8 & 5 & 0 & 0.0267 & 0 \\
\hline 9 & 10 & 0.0026 & 0.0322 & 1.2300 \\
\hline 4 & 11 & 0.0209 & 0.0688 & 0.0175 \\
\hline 5 & 11 & 0.0203 & 0.0682 & 0.0174 \\
\hline 11 & 12 & 0.0060 & 0.0196 & 0.0050 \\
\hline 2 & 12 & 0.0187 & 0.0616 & 0.0157 \\
\hline 3 & 12 & 0.0484 & 0.1600 & 0.0406 \\
\hline 7 & 12 & 0.0086 & 0.0340 & 0.0087 \\
\hline 11 & 13 & 0.0222 & 0.0731 & 0.0188 \\
\hline 12 & 14 & 0.0215 & 0.0707 & 0.0182 \\
\hline 13 & 15 & 0.0744 & 0.2444 & 0.0627 \\
\hline 14 & 15 & 0.0595 & 0.1950 & 0.0502 \\
\hline 12 & 16 & 0.0212 & 0.0834 & 0.0214 \\
\hline 15 & 17 & 0.0132 & 0.0437 & 0.0444 \\
\hline 16 & 17 & 0.0454 & 0.1801 & 0.0466 \\
\hline 17 & 18 & 0.0123 & 0.0505 & 0.0130 \\
\hline 18 & 19 & 0.0112 & 0.0493 & 0.0114 \\
\hline 19 & 20 & 0.0252 & 0.1170 & 0.0298 \\
\hline 15 & 19 & 0.0120 & 0.0394 & 0.0101 \\
\hline 20 & 21 & 0.0183 & 0.0849 & 0.0216 \\
\hline 21 & 22 & 0.0209 & 0.0970 & 0.0246 \\
\hline 22 & 23 & 0.0342 & 0.1590 & 0.0404 \\
\hline 23 & 24 & 0.0135 & 0.0492 & 0.0498 \\
\hline 23 & 25 & 0.0156 & 0.0800 & 0.0864 \\
\hline 26 & 25 & 0 & 0.0382 & 0 \\
\hline 25 & 27 & 0.0318 & 0.1630 & 0.1764 \\
\hline 27 & 28 & 0.0191 & 0.0855 & 0.0216 \\
\hline 28 & 29 & 0.0237 & 0.0943 & 0.0238 \\
\hline 30 & 17 & 0 & 0.0388 & 0 \\
\hline 8 & 30 & 0.0043 & 0.0504 & 0.5140 \\
\hline 26 & 30 & 0.0080 & 0.0860 & 0.9080 \\
\hline
\end{tabular}


Table C.5 Impedance and Line Charging Data of IEEE 118-bus system (continue)

\begin{tabular}{|c|c|c|c|c|}
\hline 17 & 31 & 0.0474 & 0.1563 & 0.0399 \\
\hline 29 & 31 & 0.0108 & 0.0331 & 0.0083 \\
\hline 23 & 32 & 0.0317 & 0.1153 & 0.1173 \\
\hline 31 & 32 & 0.0298 & 0.0985 & 0.0251 \\
\hline 27 & 32 & 0.0229 & 0.0755 & 0.0193 \\
\hline 15 & 33 & 0.0380 & 0.1244 & 0.0319 \\
\hline 19 & 34 & 0.0752 & 0.2470 & 0.0632 \\
\hline 35 & 36 & 0.0022 & 0.0102 & 0.0027 \\
\hline 35 & 37 & 0.0110 & 0.0497 & 0.0132 \\
\hline 33 & 37 & 0.0415 & 0.1420 & 0.0366 \\
\hline 34 & 36 & 0.0087 & 0.0268 & 0.0057 \\
\hline 34 & 37 & 0.0026 & 0.0094 & 0.0098 \\
\hline 38 & 37 & 0 & 0.0375 & 0 \\
\hline 37 & 39 & 0.0321 & 0.1060 & 0.0270 \\
\hline 37 & 40 & 0.0593 & 0.1680 & 0.0420 \\
\hline 30 & 38 & 0.0046 & 0.0540 & 0.4220 \\
\hline 39 & 40 & 0.0184 & 0.0605 & 0.0155 \\
\hline 40 & 41 & 0.0145 & 0.0487 & 0.0122 \\
\hline 40 & 42 & 0.0555 & 0.1830 & 0.0466 \\
\hline 41 & 42 & 0.0410 & 0.1350 & 0.0344 \\
\hline 43 & 44 & 0.0608 & 0.2454 & 0.0607 \\
\hline 34 & 43 & 0.0413 & 0.1681 & 0.0423 \\
\hline 44 & 45 & 0.0224 & 0.0901 & 0.0224 \\
\hline 45 & 46 & 0.0400 & 0.1356 & 0.0332 \\
\hline 46 & 47 & 0.0380 & 0.1270 & 0.0316 \\
\hline 46 & 48 & 0.0601 & 0.1890 & 0.0472 \\
\hline 47 & 49 & 0.0191 & 0.0625 & 0.0160 \\
\hline 42 & 49 & 0.0715 & 0.3230 & 0.0860 \\
\hline 42 & 49 & 0.0715 & 0.3230 & 0.0860 \\
\hline 45 & 49 & 0.0684 & 0.1860 & 0.0444 \\
\hline 48 & 49 & 0.0179 & 0.0505 & 0.0126 \\
\hline 49 & 50 & 0.0267 & 0.0752 & 0.0187 \\
\hline 49 & 51 & 0.0486 & 0.1370 & 0.0342 \\
\hline 51 & 52 & 0.0203 & 0.0588 & 0.0140 \\
\hline 52 & 53 & 0.0405 & 0.1635 & 0.0406 \\
\hline 53 & 54 & 0.0263 & 0.1220 & 0.0310 \\
\hline 49 & 54 & 0.0730 & 0.2890 & 0.0738 \\
\hline 49 & 54 & 0.0869 & 0.2910 & 0.0730 \\
\hline 54 & 55 & 0.0169 & \begin{tabular}{|l}
0.0707 \\
\end{tabular} & 0.0202 \\
\hline 54 & 56 & 0.0027 & 0.0095 & 0.0073 \\
\hline 55 & 56 & 0.0049 & 0.0151 & 0.0037 \\
\hline 56 & 57 & 0.0343 & 0.0966 & 0.0242 \\
\hline
\end{tabular}


Table C.5 Impedance and Line Charging Data of IEEE 118-bus system (continue)

\begin{tabular}{|c|c|c|c|c|}
\hline 50 & 57 & 0.0474 & 0.1340 & 0.0332 \\
\hline 56 & 58 & 0.0343 & 0.0966 & 0.0242 \\
\hline 51 & 58 & 0.0255 & 0.0719 & 0.0179 \\
\hline 54 & 59 & 0.0503 & 0.2293 & 0.0598 \\
\hline 56 & 59 & 0.0825 & 0.2510 & 0.0569 \\
\hline 56 & 59 & 0.0803 & 0.2390 & 0.0536 \\
\hline 55 & 59 & 0.0474 & 0.2158 & 0.0565 \\
\hline 59 & 60 & 0.0317 & 0.1450 & 0.0376 \\
\hline 59 & 61 & 0.0328 & 0.1500 & 0.0388 \\
\hline 60 & 61 & 0.0026 & 0.0135 & 0.0146 \\
\hline 60 & 62 & 0.0123 & 0.0561 & 0.0147 \\
\hline 61 & 62 & 0.0082 & 0.0376 & 0.0098 \\
\hline 63 & 59 & 0 & 0.0386 & 0 \\
\hline 63 & 64 & 0.0017 & 0.0200 & 0.2160 \\
\hline 64 & 61 & 0 & 0.0268 & 0 \\
\hline 38 & 65 & 0.0090 & 0.0986 & 1.0460 \\
\hline 64 & 65 & 0.0027 & 0.0302 & 0.3800 \\
\hline 49 & 66 & 0.0180 & 0.0919 & 0.0248 \\
\hline 49 & 66 & 0.0180 & 0.0919 & 0.0248 \\
\hline 62 & 66 & 0.0482 & 0.2180 & 0.0578 \\
\hline 62 & 67 & 0.0258 & 0.1170 & 0.0310 \\
\hline 65 & 66 & 0 & 0.0370 & 0 \\
\hline 66 & 67 & 0.0224 & 0.1015 & 0.0268 \\
\hline 65 & 68 & 0.0014 & 0.0160 & 0.6380 \\
\hline 47 & 69 & 0.0844 & 0.2778 & 0.0709 \\
\hline 49 & 69 & 0.0985 & 0.3240 & 0.0828 \\
\hline 68 & 69 & 0 & 0.0370 & 0 \\
\hline 69 & 70 & 0.0300 & 0.1270 & 0.1220 \\
\hline 24 & 70 & 0.0022 & 0.4115 & 0.1020 \\
\hline 70 & 71 & 0.0088 & 0.0355 & 0.0088 \\
\hline 24 & 72 & 0.0488 & 0.1960 & 0.0488 \\
\hline 71 & 72 & 0.0446 & 0.1800 & 0.0444 \\
\hline 71 & 73 & 0.0087 & 0.0454 & 0.0118 \\
\hline 70 & 74 & 0.0401 & 0.1323 & 0.0337 \\
\hline 70 & 75 & 0.0428 & 0.1410 & 0.0360 \\
\hline 69 & 75 & 0.0405 & 0.1220 & 0.1240 \\
\hline 74 & 75 & 0.0123 & 0.0406 & 0.0103 \\
\hline 76 & 77 & 0.0444 & 0.1480 & 0.0368 \\
\hline 69 & 77 & 0.0309 & 0.1010 & 0.1038 \\
\hline 75 & 77 & 0.0601 & 0.1999 & 0.0498 \\
\hline 77 & 78 & 0.0038 & 0.0124 & 0.0126 \\
\hline 78 & 79 & 0.0055 & 0.0244 & 0.0065 \\
\hline
\end{tabular}


Table C.5 Impedance and Line Charging Data of IEEE 118-bus system (continue)

\begin{tabular}{|c|c|c|c|c|}
\hline 77 & 80 & 0.0170 & 0.0485 & 0.0472 \\
\hline 77 & 80 & 0.0294 & 0.1050 & 0.0228 \\
\hline 79 & 80 & 0.0156 & 0.0704 & 0.0187 \\
\hline 68 & 81 & 0.0018 & 0.0202 & 0.8080 \\
\hline 81 & 80 & 0 & 0.0370 & 0 \\
\hline 77 & 82 & 0.0298 & 0.0853 & 0.0817 \\
\hline 82 & 83 & 0.0112 & 0.0367 & 0.0380 \\
\hline 83 & 84 & 0.0625 & 0.1320 & 0.0258 \\
\hline 83 & 85 & 0.0430 & 0.1480 & 0.0348 \\
\hline 84 & 85 & 0.0302 & 0.0641 & 0.0123 \\
\hline 85 & 86 & 0.0350 & 0.1230 & 0.0276 \\
\hline 86 & 87 & 0.0283 & 0.2074 & 0.0445 \\
\hline 85 & 88 & 0.0200 & 0.1020 & 0.0276 \\
\hline 85 & 89 & 0.0239 & 0.1730 & 0.0470 \\
\hline 88 & 89 & 0.0139 & 0.0712 & 0.0193 \\
\hline 89 & 90 & 0.0518 & 0.1880 & 0.0528 \\
\hline 89 & 90 & 0.0238 & 0.0997 & 0.1060 \\
\hline 90 & 91 & 0.0254 & 0.0836 & 0.0214 \\
\hline 89 & 92 & 0.0099 & 0.0505 & 0.0548 \\
\hline 89 & 92 & 0.0393 & 0.1581 & 0.0414 \\
\hline 91 & 92 & 0.0387 & 0.1272 & 0.0327 \\
\hline 92 & 93 & 0.0258 & 0.0848 & 0.0218 \\
\hline 92 & 94 & 0.0481 & 0.1580 & 0.0406 \\
\hline 93 & 94 & 0.0223 & 0.0732 & 0.0188 \\
\hline 94 & 95 & 0.0132 & 0.0434 & 0.0111 \\
\hline 80 & 96 & 0.0356 & 0.1820 & 0.0494 \\
\hline 82 & 96 & 0.0162 & 0.0530 & 0.0544 \\
\hline 94 & 96 & 0.0269 & 0.0869 & 0.0230 \\
\hline 80 & 97 & 0.0183 & 0.0934 & 0.0254 \\
\hline 80 & 98 & 0.0238 & 0.1080 & 0.0286 \\
\hline 80 & 99 & 0.0454 & 0.2060 & 0.0546 \\
\hline 92 & 100 & 0.0648 & 0.2950 & 0.0472 \\
\hline 94 & 100 & 0.0178 & 0.0580 & 0.0604 \\
\hline 95 & 96 & 0.0171 & 0.0547 & 0.0147 \\
\hline 96 & 97 & 0.0173 & 0.0885 & 0.0240 \\
\hline 98 & 100 & 0.0397 & 0.1790 & 0.0476 \\
\hline 99 & 100 & 0.0180 & 0.0813 & 0.0216 \\
\hline 100 & 101 & 0.0277 & 0.1262 & 0.0328 \\
\hline 92 & 102 & 0.0123 & 0.0559 & 0.0146 \\
\hline 101 & 102 & 0.0246 & 0.1120 & 0.0294 \\
\hline 100 & 103 & 0.0160 & 0.0525 & 0.0536 \\
\hline 100 & 104 & 0.0451 & 0.2040 & 0.0541 \\
\hline
\end{tabular}


Table C.5 Impedance and Line Charging Data of IEEE 118-bus system (continue)

\begin{tabular}{c|l|l|l|l}
\hline 103 & 104 & 0.0466 & 0.1584 & 0.0407 \\
\hline 103 & 105 & 0.0535 & 0.1625 & 0.0408 \\
\hline 100 & 106 & 0.0605 & 0.2290 & 0.0620 \\
\hline 104 & 105 & 0.0099 & 0.0378 & 0.0099 \\
\hline 105 & 106 & 0.0140 & 0.0547 & 0.0143 \\
\hline 105 & 107 & 0.0530 & 0.1830 & 0.0472 \\
\hline 105 & 108 & 0.0261 & 0.0703 & 0.0184 \\
\hline 106 & 107 & 0.0530 & 0.1830 & 0.0472 \\
\hline 108 & 109 & 0.0105 & 0.0288 & 0.0076 \\
\hline 103 & 110 & 0.0391 & 0.1813 & 0.0461 \\
\hline 109 & 110 & 0.0278 & 0.0762 & 0.0202 \\
\hline 110 & 111 & 0.0220 & 0.0755 & 0.0200 \\
\hline 110 & 112 & 0.0247 & 0.0640 & 0.0620 \\
\hline 17 & 113 & 0.0091 & 0.0301 & 0.0077 \\
\hline 32 & 113 & 0.0615 & 0.2030 & 0.0518 \\
\hline 32 & 114 & 0.0135 & 0.0612 & 0.0163 \\
\hline 27 & 115 & 0.0164 & 0.0741 & 0.0197 \\
\hline 114 & 115 & 0.0023 & 0.0104 & 0.0028 \\
\hline 68 & 116 & 0.0003 & 0.0040 & 0.1640 \\
\hline 12 & 117 & 0.0329 & 0.1400 & 0.0358 \\
\hline 75 & 118 & 0.0145 & 0.0481 & 0.0120 \\
\hline 76 & 118 & 0.0164 & 0.0544 & 0.0136 \\
\hline & & & &
\end{tabular}




\section{Appendix D}

\section{Real-Coded GA}

Initial population: The first step in real-coded genetic algorithm is to create an initial population consisting of random individuals. The function crtrp produces a matrix, Chrom, containing uniformly distributed random values in its elements.

Let's for example create a random population of 3 individuals with 4 variables. The range for every variable is defined as:

$$
\text { bounds }=\left[\begin{array}{cccc}
-100 & -50 & -30 & -20 \\
100 & 50 & 30 & 20
\end{array}\right] \begin{array}{ccc}
\% & \text { Lower bound } \\
\% & \text { Upper bound }
\end{array}
$$

Creating initial population, would yield:

$$
\text { Chrom }=\left[\begin{array}{cccc}
-72.2218 & 10.3792 & -29.0836 & 17.2726 \\
-59.4470 & -22.7812 & 14.8071 & -1.3602 \\
-60.2557 & -30.1186 & -3.2942 & -3.2540
\end{array}\right] \begin{array}{ccc}
\% & \text { parent 1 } \\
\% & \text { parent 2 } \\
\% & \text { parent 3 }
\end{array}
$$


Crossover: A discrete recombination method (equivalent to crossover) is employed for mating individuals and breeding of offsprings. Discrete recombination exchanges variable values between the individuals.

A method known as simple crossover [22,39] is implemented. Specifically, let's assume that Chrom $_{1}=\left(c_{1}^{1} \ldots c_{n}^{1}\right)$ and Chrom $_{2}=\left(c_{1}^{2} \ldots \mathcal{c}_{n}^{2}\right)$ are two chromosomes that are being subjected to crossover. A position $i \in(1,2,3, \ldots \ldots, n-1)$, where $n$ is the number of variables, is randomly assigned. The two new chromosomes are made as the following:

$$
\begin{aligned}
& \text { Chrom }_{1, \text { new }}=\left(c_{1}^{1}, c_{2}^{1}, \ldots, c_{i}^{1}, c_{i+1}^{2}, \ldots c_{n}^{2}\right) \\
& \text { Chrom }_{2, \text { new }}=\left(c_{1}^{2}, c_{2}^{2}, \ldots, c_{i}^{2}, c_{i+1}^{1}, \ldots c_{n}^{1}\right)
\end{aligned}
$$

In the package used the corssover is done through the recdis function, which intermediately produce an internal mask table determining which parents contribute which variables to the offspring, (with probability = to crossover rate), e.g. let

$$
\operatorname{mask}=\left[\begin{array}{llll}
1 & 2 & 2 & 1 \\
2 & 1 & 1 & 1 \\
0 & 0 & 0 & 0
\end{array}\right] \begin{gathered}
\% \text { for producing offspring1 } \\
\% \text { appended without recombination }
\end{gathered}
$$

Thus, after recombination NewChrom would become:

new Chrom $=\left[\begin{array}{cccc}-72.2218 & -22.7812 & 14.8071 & 17.2726 \\ -59.4470 & 10.3792 & -29.0836 & 17.2726 \\ -60.2557 & -30.1186 & -3.2942 & -3.2540\end{array}\right] \begin{array}{cc}\text { \% parent 1 \& } 2 \\ \% \text { parent 2 \& 1 } \\ \text { \% parent 3 }\end{array}$ 
Mutation: Mutation of real-valued population is accomplished with the breeder genetic algorithm in [126]. Each variable is mutated with a probability by addition of small random values (size of the mutation step). The mutation step can be reduced as the algorithm evolves.

The function mutbga produces an internal mask table, MutMask (a matrix of 0,1 and -1 , assigned with probability $=$ to mutation rate), to determine which variable to mutate and assign a sign. delta is a second internal matrix that specifies the normalized mutation step size for every variable, (see Algorithm in [126]). The mutation of a variable is then computed as follows:

mutated variable $=$ variable + MutMask $*\left(\right.$ bounds $\left.^{*} 0.5\right) \star \operatorname{delta}$

Thus, after mutation Chrom :

$$
\text { new Chrom }=\left[\begin{array}{cccc}
-72.2218 & 50.0000 & 0.9164 & -2.7274 \\
-59.4470 & -22.7812 & 14.8071 & -1.9876 \\
-60.2557 & -30.1186 & -3.2942 & -3.2735
\end{array}\right]
$$

NewChrom - Chrom shows the mutation steps 


$$
\left[\begin{array}{cccc}
0 & -39.6208 & -30.0000 & 20.0000 \\
0 & 0 & 0 & 0.6274 \\
0 & 0 & 0 & 0.0195
\end{array}\right]
$$

\section{Representation of Real Numbers in modern PC}

- A real number $n$ is represented by a floating-point approximation.

- The computer uses 32 bits (or more) to store each approximation

- It needs to store

- the mantissa

- the sign of the mantissa

- the exponent (with its sign)

The standard way to allocate 32 bits (specified by IEEE Standard 754) is:

-23 bits for the mantissa

-1 bit for the mantissa's sign 
-8 bits for the exponent

\section{Example :}

Represent -118.625 (32-bit machine)

We need to get the sign, the exponent and the fraction. Because it is a negative number, the sign is "1". Then we write the number (without the sign) using binary notation. The result is 1110110.101

Now, let's move the radix point left, leaving only a 1 at its left: $1110110.101=1.110110101 \cdot 2^{6}$

The fraction (or mantissa), is the part at the right of the radix point, filled with 0 on the right until we get all 23 bits. That is 11011010100000000000000 .

The exponent is 6, but we need to convert it to binary and bias it. For the 32-bit IEEE 754 format, the bias is 127 and so $6+127=133$. In binary, this is written as 10000101 .

- Sign: 1

- Mantissa: 11011010100000000000000

- Exponent (excess-127 format): 10000101 


\section{List of Publication}

The following publications have been derived from this thesis.

(1) A. K. AL-Othman and M. R. Irving, "Uncertainty Modeling in Power System State Estimation," IEE Proceedings on Generation. Transmission and Distribution, Accepted for publication, 2004.

(2) A. K. AL-Othman and M. R. Irving, "Analysis of Confidence Bounds in Power System State Estimation with Uncertainty in Both Measurements and Parameters," Proceedings of the 4th IASTED International Conference on Power And Energy Systems (EroPES2004), Rhodes, Greece, 2004, pp.389-393. Also submitted for review to International Journal of Power and Energy Systems.

(3) A. K. AL-Othman and M. R. Irving, "A comparative study of two methods for uncertainty analysis of power system state estimation," IEEE Transactions on Power Systems, Accepted for publication, 2004.

(4) A. K. AL-Othman and M. R. Irving, "Solving the Load-Flow Problem using Real-Coded Genetic Algorithms," Submitted for the IASTED conference on "Energy and Power Systems" (EPS 2005), 2004.

(5) M. R. Irving and A. K. Al-Othman, "Admittance matrix models of three-phase transformers with various neutral grounding configurations," IEEE Transactions on Power Systems, vol. 18, pp. 1210-1212, 2003. 


\section{References}

[1] F. C. Schweppe and J. Wildes, "Power System Static-State Estimation, Part I: Exact Model," IEEE Transactions on Power Apparatus and Systems, vol. PAS89, pp. 120-125, 1970.

[2] F. C. Schweppe and D. B. Rom, "Power System Static-State Estimation, Part II: Approximate Model," IEEE Transactions on Power Apparatus and Systems, vol. PAS-89, pp. 125-130, 1970.

[3] F. C. Schweppe, "Power System Static-State Estimation, Part III: Implementation," IEEE Transactions on Power Apparatus and Systems, vol. PAS-89, pp. 130-135, 1970.

[4] R. E. Larson, W. F. Tinney, and J. Peschon, "State Estimation in Power Systems, Part I: Theory and Feasibility," IEEE Transactions on Power Apparatus and Systems, vol. PAS-89, pp. 345-352, 1970.

[5] R. E. Larson, W. F. Tinney, L. Hajdu, and D. Piercy, "State Estimation in Power Systems, Part Il: Implementation and Applications," IEEE Transactions on Power Apparatus and Systems, vol. PAS-89, pp. 353-362, 1970.

[6] R. R. Nucera and M. L. Gilles, "A blocked sparse matrix formulation for the solution of equality-constrained state estimation," Power Systems, IEEE Transactions on, vol. 6, pp. 214 - 224, 1991.

[7] F. F. Wu, W.-H. E. Liu, and S.-M. Lun, "Observability analysis and bad data processing for state estimation with equality constraints," Power Systems, IEEE Transactions on, vol. 3, pp. 541 - 548, 1988.

[8] K. A. Clements, P. W. Davis, and K. D. Frey, "Treatment of inequality constraints in power system state estimation," Power Systems, IEEE Transactions on, vol. 10, pp. 567 - 574, May 1995.

[9] H. Singh, F. L. Alvarado, and W.-H. E. Liu, "Constrained LAV state estimation using penalty functions," Power Systems, IEEE Transactions on, vol. 12, pp. 383 - 388, Feb. 1997. 
[10] K. A. Clements, G. W. Woodzell, and R. C. Burchett, "A new method for solving equality-constrained power system static-state estimation," Power Systems, IEEE Transactions on, vol. 5, pp. 1260 - 1266, Nov. 1990.

[11] A. Abur and M. K. Celik, "Least absolute value state estimation with equality and inequality constraints," Power Systems, IEEE Transactions on, vol. 8, pp. 680 686, May 1993.

[12] M. Irving, R. Owen, and M. Sterling, "Power system state estimation using linear programming," Proceedings of the Institution of Electrical Engineers, vol. 125, pp. 879 -885, 1978.

[13] "Final Report on the August 14, 2003 Blackout in the United States and Canada: Causes and Recommendations," U.S.-Canada Power System Outage Task Force, April 2004.

[14] A. Bargiela and G. Hainsworth, "Pressure and flow uncertainty in water system," ASC J. Water Resources Planning Mang, vol. 115 (2), pp. 212 -229, 1989.

[15] "Expression of the Uncertainty of Measurement in Calibration," EAL-4/02, Dec 1999.

[16] J. B. Ward and H. W. Hale, "Digital computer solution of power-flow problems," Trans. AIEE, vol. PAS-75, pp. 938-404, 1956.

[17] A. R. Bergen and V. Vittal, Power systems analysis, 2nd ed. Upper Saddle River, N.J.: Prentice Hall, 2000.

[18] J. D. Glover and M. S. Sarma, Power system analysis and design, 2nd ed. Boston: PWS Pub., 1994.

[19] J. J. Grainger and W. D. Stevenson, Power system analysis. New York: McGrawHill, 1994.

[20] G. T. Heydt, Computer analysis methods for power systems. New York: Macmillan Pub. Co., 1986.

[21] D. E. Goldberg, Genetic algorithms in search, optimization, and machine learning. Reading, Mass. ; Harlow: Addison-Wesley, 1989.

[22] Z. Michalewicz, Genetic algorithms + data structures $=$ evolution programs, 3rd rev. and extended ed. Berlin ; New York: Springer-Verlag, 1996. 
[23] V. Miranda, D. Srinivasan, and L. M. Proenca, "Evolutionary computation in power systems," International Journal of Electrical Power \& Energy Systems, vol. 20, pp. 89-98, 1997.

[24] V. Miranda, "Evolutionary computation in power systems," Fuel and Energy Abstracts, vol. 39, pp. 227, 1998.

[25] X. D. Yin and N. Germay, "Investigation on Solving the Load Flow Problem by Genetic Algorithms," Elect. Power Systems Res, vol. 22, pp. 151-163, 1991.

[26] K. P. Wong, A. Li, and T. M. Y. Law, "Advanced constrained genetic algorithm load flow method," Generation, Transmission and Distribution, IEE Proceedings, vol. 146, pp. 609 -616, Nov. 1999.

[27] K. P. Wong and A. Li, "Solving the load-flow problem using genetic algorithm," Evolutionary Computation, 1995., IEEE International Conference on, vol. 1, pp. 103, 29 Nov.-1 Dec. 1995.

[28] K. P. Wong, A. Li, and M. Y. Law, "Development of constrained-geneticalgorithm load-flow method," Generation, Transmission and Distribution, IEE Proceedings-, vol. 144, pp. 91 -99, March 1997.

[29] F. Herrera, M. Lozano, and J. L. Verdegay, "Tackling Real-Coded genetic Algorithms: operators and Tools for Behavioral Analysis," Artificial Intelligence Review, vol. 12, pp. 265-319, 1998.

[30] C. Z. Janikow and Z. Michalewicz, "An Experimental Comparison of Binary and Floating Point Representations in Genetic Algorithms," Proceedings of 4th Intl. Conference on Genetic Algorithms, 1991, pp.31-36.

[31] S.Abe, N. Hamada, A. Isono, and K. Okuda, "Load Flow Convergence in the Vicinity of a Voltage Stability Limit," IEEE Trans. Power Apparatus and Systems, vol. PAS-97, pp. 1983-1993, Nov/Dec 1978.

[32] K. Iba, H. Suzuki, M. Egawa, and T. Watanabe, "A method for finding a pair of multiple load flow solutions in bulk power systems," Power Systems, IEEE Transactions on, vol. 5, pp. 582 -591, May 1990. 
[33] X. Yin, "Application of genetic algorithms to multiple load flow solution problem in electrical power systems," Proceedings of IEEE Conference on Decision and Control, Proceedings of the 32nd, 1993, pp.3734 -3739.

[34] E. Falkenauer, Genetic algorithms and grouping problems. New York: Wiley, 1997.

[35] I. G. Damousis, A. G. Bakirtzis, and P. S. Dokopoulos, "Network-constrained economic dispatch using real-coded genetic algorithm," Power Systems, IEEE Transactions on, vol. 18, pp. 198 - 205, Feb. 2003.

[36] S. Baskar, P. Subbaraj, and M. V. C. Rao, "Hybrid real coded genetic algorithm solution to economic dispatch problem," Computers \& Electrical Engineering, vol. 29, pp. 407-419, 2003.

[37] D. Whitley, "The GENITOR Algorithm and Selection Pressure: Why Rank-Based Allocation of Reproductive Trials is Best," Proceedings of Proc. ICGA 3, 1989, pp.116-121.

[38] J. E. Baker, "Reducing bias and inefficiency in the selection algorithm," Proceedings of ICGA, 1987, pp.14-21.

[39] A. H. Wright, "Genetic algorithms for real parameter optimization," Proceedings of Foundations of Genetic Algorithms,(edited by Gregory J. E. Rawlins), Morgan Kaufman, 1991, pp.205-218.

[40] H. Mühlenbein and D. Schlierkamp-Voosen, "Predictive Models for the Breeder Genetic Algorithm: I. Continuous Parameter Optimization," Evolutionary Computation, vol. 1, pp. 25-49, 1993.

[41] A. J. Wood and B. F. Wollenberg, Power generation, operation, and control, 2nd ed. New York ; Chichester: John Wiley \& Sons, 1996.

[42] F. C. Schweppe, Uncertain dynamic systems. Englewood Cliffs, N.J.: PrenticeHall, 1973.

[43] M.Brdys and K. Chen, "joint state and parameter estimation of dynamic water supply systems under bounded uncertainty," Proceedings of 1993 Integ. computer application in water supply, 1993, pp. 335 -353. 
[44] J.Andersen, R.Powell, and J.Marsh, "Constrained state estimation with application in water distribution network monitoring," Int. J. of systems \& Sci, vol. 32, pp. 807-816, 2001.

[45] A.Bjorck, Numerical Methods for Least Squares Problems. Philadelphia: SIAM, 1996.

[46] B. Kolman, Introductory linear algebra with applications, 4th ed. New York, London: Macmillan ; Collier Macmillan, 1988.

[47] A. Monticelli, State estimation in electric power systems : a generalized approach. Boston ; London: Kluwer Academic Publishers, 1999.

[48] A. Abur and A. G. Exposito, Power System State Estimation: Theory and Implementation: Marcel Dekker, 2004.

[49] "Optimization Toolbox for use with Matlab user's guide," 2 ed: The Math works inc.

[50] W.Pedrycz and A.Bargiela, Granular Computing: An Introduction. Boston: Kuler, 2002.

[51] A. Bargiela, W. Pedrycz, and M. Tanaka, "A study of uncertain state estimation," Systems, Man and Cybernetics, Part A, IEEE Transactions on, vol. 33, pp. 288 301, May 2003.

[52] A. El-Kei and H. Singh, "Fast linear programming state estimation using the dual formulation," IEEE Transactions on. Power Systems, vol. 7, pp. 620 -628, May 1992.

[53] V. Chvatal, Linear Programming.: Freeman, 1983.

[54] R. Vanderbei, Linear Programming: Foundations and Extensions. Boston: Kluwer, 2002.

[55] M. M. Adibi and D. K. Thorne, "Remote measurement calibration," Power Systems, IEEE Transactions on, vol. PWRS-1, pp. 194-202, May 1986.

[56] M. M. Adibi, K. A. Clements, R. J. Kafka, and J. P. Stovall, "Remote measurement calibration," IEEE Computer Applications in Power, vol. 3, pp. 37 42 , Oct. 1990. 
[57] H. I. Anis, S. A. Mahmoud, and M. A. Abdallah, "Probabilistic modeling of power lines magnetic fields," Proceedings of Power Engineering Society Summer Meeting, 2000, 16-20 July 2000, pp.2383 -2387.

[58] A. Polevoy, "Calculation of sag changes caused by conductor beating with consideration of insulator string deviation in a transmission line section," Power Delivery, IEEE Transactions on, vol. 13, pp. 1238 -1243, Oct. 1998.

[59] G.H.Golub and C. V. Loan, Matrix computations, thiard ed: Johns Hopkins University Press, 1996.

[60] C. L. Lawson and R. J. Hanson, Solving Least-Squares Problems: SIAM, Philadelphia, PA, 1995.

[61] A. H. Sayed, V. H. Nascimento, and S. Chandrasekaran, "Estimation and control with bounded data uncertainties," Linear Algebra and its Applications, vol. 284, pp. 259-306, 1998.

[62] G. H. Golub and C. F. V. Loan, "An analysis of the total least squares problem," SIAM Journal on Numerical Analysis, vol. 17, pp. 883--893, 1980.

[63] S. Chandrasekaran, G. H. Golub, M. Gu, and A. H. Sayed, "Parameter Estimation in the Presence of Bounded Data Uncertainties," SIAM Journal on Matrix Analysis and Applications, vol. 19, pp. 235-252, 1998.

[64] J. B. Rosen, H. Park, and a. J. Glick, "Total Least Norm Formulation and Solution for Structured Problems," SLAM Journal on Matrix Analysis and Applications, vol. 17, pp. 110-126, 1999.

[65] A.Bargiela, "Ellipsoid method for quantifying the uncertainty in water system state estimation," Proceedings of IEE Colloquium on identification of Uncertain Systems, 1994, pp.10/1 - 10/3.

[66] A. Nagar and R. Powell, "LFT/SDP approach to the uncertainty analysis for state estimation of water distribution systems," IEE Proceedings on. Control Theory and Applications, vol. 149, pp. 137 -142, Mar 2002.

[67] K. Zhou, J. C. Doyle, and K. Gloverl, Robust and Optimal Contro: Prentice-Hall, Englewood Cliffs, NJ, 1996. 
[68] L. El Ghaoui and G. Calafiore, "Worst-case state prediction under structured uncertainty," Proceedings of American Control Conference, 1999, pp.3402 3406.

[69] J. G. VanAntwerp and R. D. Braatz, "A tutorial on linear and bilinear matrix inequalities," Journal of Process Control, vol. 10, pp. 363-385, 2000.

[70] L. El Ghaoui and G. Calafiore, "Confidence ellipsoids for uncertain linear equations with structure," Proceedings of 38th IEEE Conference on Decision and Control, 1999, pp.1922 - 1927.

[71] M. J. D. Powell, "Introduction to constrained optimization," in Numerical methods for constrained optimization: Academic Press, 1974, pp. 1-28.

[72] M. J. D. Powell, A Fast Algorithm for Nonlinearly Constrained Optimization Calculations, vol. 630: Numerical Analysis, ed. G.A.Watson, Lecture Notes in Mathematics,Springer Verlag, 1978.

[73] M. J. D. Powell, "A fast algorithm for nonlinearly constrained optimization calculations," Proceedings of Proceedings of the Biennial Conference on numerical analysis, 28 June-1 July 1977, Dundee, UK, 1978, pp.144-57.

[74] N. H. Abbasy and S. M. Shahidehpour, "Application of Non-Linear Programming in Power System State Estimation," Electric Power Systems Research, vol. 11, pp. 463-472, 1987.

[75] M. J. D. Powell, "A note on quasi-Newton formulae for sparse second derivative matrices," Mathematical Programming, vol. 20, pp. 144-51, 1981.

[76] M. J. D. Powell, "Convergence properties of algorithms for nonlinear optimization," SIAM Review, vol. 28, pp. 487-500, 1986.

[77] Y. Wallach, Calculations and programs for power system networks. Englewood Cliffs, N.J.: Prentice-Hall, 1986.

[78] P. J. Rousseeuw, "Least median of squares regression," Journal of the American Statistical Association, vol. 79, pp. 871-880, 1984.

[79] P. J. Rousseeuw and A. M. Leroy, Robust regression and outlier detection. New York ; Chichester: Wiley, 1987. 
[80] L. Mili, V. Phaniraj, and P. J. Rousseeuw, "Least median of squares estimation in power systems," Power Systems, IEEE Transactions on, vol. 6, pp. 511 -523, 1991.

[81] L. Mili, M. G. Cheniae, N. S. Vichare, and P. J. Rousseeuw, "Algorithms for least median of squares state estimation of power systems," Proceedings of Circuits and Systems, Proceedings of the 35th Midwest Symposium on, 1992, pp.1276 1283.

[82] L. Mili, M. G. Cheniae, N. S. Vichare, and P. J. Rousseeuw, "Robust state estimation based on projection statistics of power systems," Power Systems, IEEE Transactions on, vol. 11, pp. 1118 -1127, 1996.

[83] L. Mili, N. S. Vichare, M. G. Cheniae, and P. J. Rousseeuw, "Robust Mahalanobis distances in power system state estimation," Proceedings of 36th Midwest Symposium on Circuits and Systems, Aug 1993, pp.1014 - 1018.

[84] N. Logic, E. Kyriakides, and G. T. Heydt, "Lp State Estimators for Power Systems," Journal of Electric Power Components and Systems, accepted for publication, 2002.

[85] L. Mili, V. Phaniraj, and P. J. Rousseeuw, "High breakdown point estimation in electric power systems," Proceedings of Circuits and Systems, IEEE International Symposium on, 1990, pp.1843-1846.

[86] A. Abur, F. H. Magnago, and F. L. Alvarado, "Elimination of leverage measurements via matrix stretching," International Journal of Electrical Power \& Energy Systems, vol. 19, pp. 557-562, 1997.

[87] F. Y. Edgeworth, "On Observations Relating to Several Quantities," Hermathena, vol. 6, pp. 279-285, 1887.

[88] W. W. Kotiuga and M. Vidyasagar, "Bad data rejection properties of weighted least absolute value techniques applied to static state estimation," IEEE Transactions on Power Apparatus and Systems, vol. PAS-101, pp. 844-851, 1982.

[89] D. M. Falcao and S. M. de Assis, "Linear programming state estimation: Error analysis and gross error identification," IEEE Transactions on Power Systems, vol. 3, pp. 809-815, 1988. 
[90] G. Christensen, A. Rouhi, and S. Soliman, "A new technique for unconstrained and constrained linear LAV parameter estimation," Canadian Journal of electrical and computer engineering, vol. 14, pp. 24-30, 1989.

[91] S. Soliman, G. Christensen, and A. Rouhi, "A new technique for curve fitting based on minimum absolute deviations," Journal of Computational Statistics \& Daia Analysis, vol. 6, pp. 341-351, 1988.

[92] P. Ryan Thomas, Modern regression methods. New York ; Chichester: J. Wiley, 1997.

[93] F. R. Hampel, Robust statistics : the approach based on influence functions. New York ; Chichester: Wiley, 1986.

[94] J. L. Hodges, "Efficiency in Normal Samples and Tolerance of Extreme Values for Some Estimates of Location," Proceedings of Fifth Berkeley Sym. Matt. Stat. Probab., Berkeley, USA, 1967, pp.163-168.

[95] F. R. Hampel, "A general qualitative definition of robustness," in Ann. Math. Stat, vol. 42, 1971, pp. 1887-1896.

[96] D. L. Donoho and P. J. Huber, "The notion of breakdown point," in P.J. Bickel, $K$. Doksum and J.L. Hodges Jr. Wadsworth, Belmont, CA: Festschrift fur Erich L. Lehmann, 1983, pp. 157-184.

[97] W. Rudin, Real and Complex Analysis, 3rd ed. New York: McGraw-Hil, 1987.

[98] J. Huber Peter, Robust statistics. New York ; Chichester: Wiley, 1981.

[99] P. J. Bickel, "On Some Analogues to Linear Combination of Order Statistics in the Linear Model," Annals of Statistics, vol. 1, pp. 597-616, 1973.

[100] L. A. Jaeckel, "Estimating Regression Coefficients by Minimizing the Dispersion of Residuals," Annals of Mathematical Statistics, vol. 5, pp. 1449-1458, 1972.

[101] P. J. Rousseeuw and V. J. Yohai, "Robust regression by means of S-estimators," in Robust and Nonlinear Time Series Analysis, Franke, J., HKardle, W., Martin. R.D. (Eds.), Lecture Notes in Statistics, vol. 26. New York: pp. 256-272,Springer, 1984.

[102] J. R. Berrendero, "On the global robustness of generalized S-estimators," Journal of Statistical Planning and Inference, vol. 102, pp. 287-302, 2002. 
[103] V. J. Yohai and R. H. Zamar, "High breakdown point estimates of regression by means of the minimization of an efficient scale," Journal of the American Statistical Association, vol. 83, pp. 406-413, 1988.

[104] K. L. Lo, P. L. Zeng, E. Marchand, and A. Pinkerton, "New bad-data detection and identification technique based on rotation of measurement order for sequential state estimation [for power systems]," IEE Proceedings $C$ (Generation, Transmission and Distribution), vol. 139, pp. 387-401.

[105] G. Dallal, "LMS: Least Median of Squares Regression," The American Statistician, vol. 45, 1991.

[106] A. Leontitsis and J. Pange, "Statistical significance of the LMS regression," Mathematics and Computers in Simulation, vol. 64, pp. 543-547, 2004.

[107] Y.-Z. Liang and O. M. Kvalheim, "Robust methods for multivariate analysis -- a tutorial review," Chemometrics and Intelligent Laboratory Systems, vol. 32, pp. 1$10,1996$.

[108] F. R. Hampel, "Beyond location parameters: Robust concepts and methods," Bull. Int'. Stat. Inst, vol. 46, pp. 375-382, 1975.

[109] L. Mili, M. G. Cheniae, and P. J. Rousseeuw, "Robust state estimation of electric power systems," Circuits and Systems I: Fundamental Theory and Applications, IEEE Transactions on, vol. 41, pp. 349 -358, May 1994.

[110] P. Rousseeuw and B. v. Zomeren, "Unmasking multivariate outliers and leverage points," Journal of the American Statistical Association, vol. 85, pp. 633-639, 1990.

[111] P. Rousseeuw and B. v. Zomeren, "Rejoinder to: Unmasking multivariate outliers and leverage points," Journal of the American Statistical Association, vol. 85, pp. 648-651, 1990.

[112] P. J. Rousseeuw, "Multivariate Estimation With High Breakdown Point," in Mathematical Statistics and Applications, edited by W. Grossmann, G. Pflug, I. Vincze, and W. Werty, Reidel, Dordrecht, vol. B. Netherlands, 1985, pp. 283-297.

[113] H. S. M. Coxeter, L. Greitzer Samuel, and A. Mathematical Association of, Geometry revisited. Washington: Mathematical Association of America, 1967. 
[114] A. Stefanski, "A note on High- Breakdown Estimator," Statistics and Probability, vol. 11, pp. 353-358, 1991.

[115] T. Hettmansperger and S. Sheather, "A Cautionary Note on the Method of Least Median Squares," Journal of the American Statistical Association, vol. 46, pp. 7983, 1992.

[116] A. Monticelli, F. Wu, and M. Yen, "Multiple bad data identification for state estimation by combinatorial optimisation," Power Delivery, IEEE Transactions on, vol. PWRD-1, No.3, pp. 361-369, 1996.

[117] A. Monticelli and A. Garcia, "Reliable bad data processing for real-time sate estimation," Power Apparatus and Systems, IEEE Transactions on, vol. PAS-102, pp. 1126-1139, 1983.

[118] M. Arakawa and I. Hagiwara, "Development of Adaptive Real Range (ARRange) Genetic Algorithms," JSME International Journal, vol. 41, pp. 969-977, 1998.

[119] A. Oyama, S. Obayashi, and T. Nakamura, "Real-coded adaptive range genetic algorithm applied to transonic wing optimization," Applied Soft Computing, vol. 1, pp. 179-187, 2001.

[120] G. Mauris, L. Berrah, L. Foulloy, and A. Haurat, "Fuzzy handling of measurement errors in instrumentation," Instrumentation and Measurement, IEEE Transactions on, vol. 49, pp. Pages:89 - 93, 2000.

[121] G. Mauris, V. Lasserre, and L. Foulloy, "A fuzzy approach for the expression of uncertainty in measurement," Measurement, vol. 29, pp. 165-177, 2001.

[122] J. Arrillaga and C. P. Arnold, Computer Analysis of Power Systems: John Wiley, 1990.

[123] J. Arrillaga and N. R. Watson, Computer Modelling of Electrical Power Systems, 2nd ed: John Wiley, 2001.

[124] W. E. Dillon and M. S. Chen, "Transformer modelling in unbalanced three-phase networks," IEEE Summer Power Meeting, Vancouver, 1972.

[125] J. Arrillaga, N. R. Watson, and S. Chen, Power System Quality Assessment: John Wiley, 2000. 
[126] H. Mühlenbein and D. Schlierkamp-Voosen, "Predictive Models for the Breeder Genetic Algorithm: I. Continuous Parameter Optimization," Evolutionary Computation, vol. 1, pp. 25-49, 1993. 